\title{
The Hyperanalytic Wavelet Transform
}

\author{
Imperial College Statistics Section \\ Technical Report TR-06-02 \\ February 2, 2008 \\ Sofia C. Olhede \& Georgios Metikas \\ Department of Mathematics, Imperial College London, SW7 2AZ UK
}

\begin{abstract}
In this paper novel classes of 2-D vector-valued spatial domain wavelets are defined, and their properties given. The wavelets are 2-D generalizations of 1-D analytic wavelets, developed from the Generalized Cauchy-Riemann equations and represented as quaternionic functions. Higher dimensionality complicates the issue of analyticity, more than one 'analytic' extension of a real function is possible, and an 'analytic' analysis wavelet will not necessarily construct 'analytic' decomposition coefficients. The decomposition of locally unidirectional and/or separable variation is investigated in detail, and two distinct families of hyperanalytic wavelet coefficients are introduced, the monogenic and the hypercomplex wavelet coefficients. The recasting of the analysis in a different frame of reference and its effect on the constructed coefficients is investigated, important issues for sampled transform coefficients. The magnitudes of the coefficients are shown to exhibit stability with respect to shifts in phase. Hyperanalytic 2-D wavelet coefficients enable the retrieval of a phase-and-magnitude description of an image in phase space, similarly to the description of a 1-D signal with the use of 1-D analytic wavelets, especially appropriate for oscillatory signals. Existing 2D directional wavelet decompositions are related to the newly developed framework, and new classes of mother wavelets are introduced.
\end{abstract}

\section{Index Terms}

Phase and frequency, image analysis, wavelet transform, wavelets, image representations, Hilbert transform.

\section{INTRODUCTION}

$\mathbf{T}$ HIS paper constructs new classes of 2-D vector-valued mother wavelet functions. The vector-valued wavelet functions are defined to produce 2-D extensions of 1-D analytic complex wavelet coefficients, denoted hyperanalytic wavelet coefficients. The extension of 1-D analytic wavelet coefficients to 2-D is non-trivial. Firstly more than one definition of 'analytic' exists in 2-D. The suitable choice of the concept in 2-D depends on the local directionality and dimensionality of the signal under analysis, and local unidirectionality, as well as separability, will explicitly be considered. Secondly, unlike the 1-D case, a distinction can be made between wavelet coefficients obtained from using wavelets that are 'analytic', wavelet coefficients that are 'analytic' in their spatial index, and wavelet coefficients of 'analytic' 2-D functions. In 2-D it will furthermore become necessary to introduce the notion of $\theta$-'analytic', or 'analytic' in a rotated frame of reference, to interpret the properties of the wavelet coefficients. We shall demonstrate that it is possible to choose a definition of 'analytic' suitably so that the hyperanalytic wavelet coefficients have tractable and useful properties in the rotated frame. The mathematical framework developed for the construction of the hyperanalytic wavelet coefficients also provides a context to some of the most promising new complex/vector-valued wavelet filter decompositions in the literature [1], [2], as well as allows us to construct new classes of vector-valued wavelets.

Over twenty year ago, Grossman and Morlet [3] developed the Continuous Wavelet Transform (CWT) [4], using continuous complex-valued mother wavelets. Initial analysis based on wavelet decompositions was implemented using such mother wavelets. Both magnitude and phase descriptions of non-stationary signals were determined, and an early example of analysis include wavelet ridge methods proposed by Delprat et al. [5]. However subsequently for many years interest focused on the Discrete Wavelet Transform (DWT) and signal estimation. The DWT was

Manuscript received XXXXXXX XX, XXXX; revised XXXXXXX XX, XXXX. G. Metikas was supported by an EPSRC grant.

G. Metikas and S. Olhede are with the Department of Mathematics, Imperial College London, SW7 2AZ, London, UK (s.olhede@imperial.ac.uk). Tel: +44 (0) 207594 8568, Fax: +44 (0) 2075948517. 
developed to implement the wavelet transform of time-compact mother wavelets and as compact discrete wavelet filters cannot be exactly analytic [6], real wavelets were used. A revival of interest in later years has occurred in both signal processing and statistics for the usage of complex wavelets [1], [7], and in particular complex analytic wavelets [8]-[11]. This revival of interest may be linked to the development of complex-valued discrete wavelet filters [12] and the clever dual filter bank [8]. The complex wavelet transform has been shown to provide a powerful tool in signal and image analysis [1], where most of the properties of the transform follow from the analyticity of the wavelet function. This paper strives to build upon the strengths of recent developments, by deriving large classes of wavelets generalizing the concept of a 1-D local complex-valued analytic decomposition to a 2-D vector-valued hyperanalytic decomposition.

The wavelet decomposition is in 2-D only a single example of a local decomposition. Local decompositions of an image in terms of spatial structure associated with a given scale and spatial position have with great success formed the basis for many different procedures of local image analysis and estimation [4], [13], [14]. As the variational structure in 2-D is much richer than in 1-D, the term 'local' may denote a variety of different forms of spatially limited structures, and so examples of decompositions include i.e. wavelets [4], curvelets [15] and bandelets [16] etc. A local decomposition method should be chosen with easy interpretability of the decomposition coefficients and compression in mind, as both of these characteristics facilitate the analysis of observed images. For local image analysis, the local directionality and dimensionality of variation are key features of the local visual appearance of the image. These two features need to be characterised, and can be used for further processing, such as image segmentation, feature detection and disparity estimation, see for example work by von Bülow and Sommer [17], as well as that of von Bülow [18], Hwang et al. [19], Antoine et al. [20], [21] etc. To represent both local dimensionality and directionality at each spatial point more than a scalar-valued representation is required. Based on a fixed local dimensionality the characterisation of the image will of course vary. For example, images that are locally 1-D, are well characterised by the direction of the image variation, its magnitude and the scale of variation in the given direction. Texture features present in an image may correspond to locally separable structures, characterised by the direction of the separable structure, the local magnitude of variation and the scale of variation in two directions. Single texture components often correspond to variations associated with the same scale in both perpendicular directions.

The observational axis of an image cannot be assumed to coincide with the natural axis of the local structure. It is therefore necessary that the local representations should not substantially change form with rotations and translations of the spatial index, but exhibit equivariance to such operations. Of course the CWT exhibits equivariance when calculated in continuous space at all values of the locality index [13], but if the transformation is to be discretized, it needs to satisfy some further constraints so that the representation is stable. In particular we require that the magnitude of the vector-valued decomposition should be stable to shifts in phase of the signal, or a sampled collection of coefficients of the process may exhibit high translation variance in comparison to a sampled collection of coefficients obtained from the same image subjected to a small spatial shift in both axes simultaneously [1], [22]. Translation variance is clearly an undesirable feature of a decomposition. The simple characterisation of any shift in index is also important for analysis of multiple images. Vector-valued representations, as will be demonstrated, can with a suitable choice of decomposition filter, both provide decompositions stable to small spatial shifts (or phase shifts) and allow for the easy characterisation of rotations and translations of the spatial index.

As examples of successful existing vector-valued local representations of image structure we note the 2-D analytic signal [23], or the complex CWT [1], [22]. The 1-D analytic signal at a given value of $x_{1}$ provides a meaningful magnitude and phase description of a signal, if only a single oscillatory component is present. For each fixed scale the complex wavelet coefficients correspond to spatially local representations of the image in terms of two wavelet coefficients, but the magnitude and phase of the complex wavelet coefficients are not when an arbitrary complex-valued wavelet is used either meaningful nor are they interpretable. For this reason complex wavelet transform coefficients obeying suitable conditions are generally used [1], [24]. We aim to generalise the usage of spatially local two-vector representations to local three- and four-vector representations, using hyperanalytic wavelet decompositions.

Vector-valued representations of local structure in terms of quaternionic representations, or equivalently threeand four-vectors, have been developed by von Bülow and Sommer [17], Felsberg and Sommer [25] as well as Hahn and Snopek [26]. In particular Hahn [27], von Bülow and Sommer [17], as well as Felsberg and Sommer [25], have developed 2-D extensions of the 1-D analytic signal. These representations give descriptions of local variation 
in terms of a local magnitude and phase/s. Phase should represent local variations and structure, whilst magnitude is usually interpreted as local signal presence [28]. A local well-constructed magnitude and phase description of a signal is often very informative. However if more than a single narrowband component is present at a given spatial location in an image, the 2-D 'analytic' signal, no matter which of the above generalizations are chosen, will completely lack interpretation, just like in the case of the 1-D analytic signal of a multi-component signal [29], [30].

The work by Hahn and Snopek [26] consists of forming a suitable 2-D quaternionic (four-vector valued) extension of the Wigner-Ville distribution [30]. In 1-D the Wigner-Ville distribution is known to be uninformative when describing signals contaminated by noise [31], and is unsuitable as a description of signals consisting of more than a single component at any spatial position due to interference between components [30]. To deal both with the addition of noise and the presence of multiple components in 1-D, local analytic representations have been constructed, such as analytic wavelet coefficients [5], [32], [33], or other local representations of the signal calculated in conjunction with a localisation procedure [34], [35]. In this paper we aim to construct 2-D 'analytic wavelet coefficients' or, locally 'analytic' representations of images, by using vector-valued wavelet functions. The wavelets will be constructed to merge the 2-D wavelet transform with existing 2-D 'analytic' representations. The synthesis of local image representations and magnitude and phase descriptions of local structure will be shown to enjoy many special properties, but requires several non-trivial generalizations of existing 1-D concepts.

Before proceeding to the construction of 2-D wavelets to serve the same purpose as 1-D analytic wavelets, let us first briefly revisit the properties of the 1-D analytic wavelet decomposition. The decomposition is constructed from a single mother wavelet function $\psi\left(x_{1}\right) \in L^{2}(\mathbb{R})$, that satisfies the admissibility condition [13]. The mother wavelet is translated in time by $b$, and scaled by factor $a$ to form a family of wavelets denoted by $\left\{\psi_{a, b}\left(x_{1}\right)\right\}$. For $\psi(\mathbf{x}) \in L^{2}\left(\mathbb{R}^{d}\right)$, the dilation and scaling operators for any index dimension $d$ are $\mathcal{D}_{a} \psi(\mathbf{x})=|a|^{-d / 2} \psi(\mathbf{x} / a)$, and $\mathcal{T}_{\mathbf{b}} \psi(\mathbf{x})=\psi(\mathbf{x}-\mathbf{b})$, respectively. A member of the 1-D wavelet family is $\psi_{a, b}\left(x_{1}\right)=\mathcal{D}_{a} \mathcal{T}_{b} \psi\left(x_{1}\right)$. To represent a 1-D signal $g(x)$ in terms of local contributions the CWT, and its inversion, are defined by

$$
w_{\psi}(a, b ; g)=\int_{-\infty}^{\infty} g\left(x_{1}\right) \psi_{a, b}^{*}\left(x_{1}\right) d x_{1}, \quad g\left(x_{1}\right)=\frac{1}{c_{\psi}} \int_{-\infty}^{\infty} \int_{-\infty}^{\infty} w_{\psi}(a, b ; g) \psi_{a, b}\left(x_{1}\right) \frac{d a}{a^{2}} d b .
$$

$c_{\psi} \in \mathbb{R}$ is a constant specific to the function $\psi\left(x_{1}\right)$, its existence guaranteed by the admissibility condition. If $\psi(\cdot)$ can be interpreted as local in time to $x_{1}=0$ and frequency to $f_{1}=f_{0}$, the signal is reconstructed in terms of the local contributions $w_{\psi}(a, b ; g)$ : furthermore $w_{\psi}(a, b ; g)$ is associated with a function local to position $x_{1}=b$ and frequency $f_{1}=f_{0} / a$. The wavelet coefficients are used to characterise the local properties of the signal and the decomposition is often described as a 'local Fourier' transform, even if this is a somewhat inaccurate description.

If $\psi\left(x_{1}\right)$ is real-valued, then the magnitude of $w_{\psi}(a, b ; g)$ can be used to determine local signal content, or be used as the basis of more complicated analysis methods. If $w_{\psi}(a, b ; g)$ is complex-valued then it can be represented by its magnitude and phase. If $\psi\left(x_{1}\right)$ is analytic, denoting an analytic mother wavelet ${ }^{1}$ by $\psi^{(+)}\left(x_{1}\right)$, then the wavelet is a complex-valued function. The CWT of a real-valued signal using a complex-valued analytic wavelet yields complex-valued wavelet coefficients, where the coefficients are an analytic signal in $b$. In 1-D, if we define the complex variable $t_{1}=x_{1}+\boldsymbol{j} y_{1}$ and restrict $y_{1}>0$, then an analytic signal $g^{(+)}\left(x_{1}\right)=g\left(x_{1}\right)+\boldsymbol{j} g^{(1)}\left(x_{1}\right)$, evaluated in the complex argument, that is $g^{(+)}\left(t_{1}\right)$, is an analytic function in the upper half-plane of $y_{1}>0$ [23]. $g^{(+)}\left(t_{1}\right)$ thus satisfies the Cauchy-Riemann equations in the upper half-plane and this determines the relationship between $g\left(t_{1}\right)$ and $g^{(1)}\left(t_{1}\right)$. The fact that a real and imaginary part of $g^{(+)}\left(x_{1}+\boldsymbol{j} y_{1}\right)$ satisfy the Cauchy-Riemann equations imply that the phase of $g^{(+)}\left(x_{1}\right)$ will be well defined, once some additional restrictions are placed on $g\left(x_{1}\right)$. Given the analytic wavelet coefficients are an analytic signal, and local in frequency, the instantaneous frequency of an oscillatory component present at scale $a$, may for example be determined using its analytic wavelet coefficients, and multi-component oscillatory signals may be characterised via wavelet ridge analysis [5]. Note that if $w_{\psi}(a, b ; g) \in \mathbb{C}$ but $\psi\left(x_{1}\right) \neq \psi^{(+)}\left(x_{1}\right)$, then $w_{\psi}(a, b ; g)$ can still be represented in terms of a magnitude and phase, but the phase will not in general be interpretable in terms of local structure. Furthermore wavelet coefficients constructed from analytic mother wavelets have a magnitude invariant to phase-shifts and this alleviates observed problems with coefficients exhibiting strong shift-dependence [1], [36]. The analytic wavelet has one major drawback, longer essential time-support, but provides the framework for analysis shorter time-support filters may

\footnotetext{
${ }^{1}$ An 'analytic' mother wavelet in this context is a complex-valued mother wavelet that is also an analytic signal [30].
} 
approximately provide, see for example work by Selesnick et al. [1] as well as Fernandes et al. [24]. Selesnick et al. give a comprehensive discussion of the many important properties of analytic wavelet coefficients when calculated in both 1-D and higher dimensions, and these properties justify our interest in developing a single mathematical framework for the interpretation of 2-D locally 'analytic' representations.

In 2-D the CWT of $g(\mathbf{x}) \in L^{2}\left(\mathbb{R}^{2}\right)$ is implemented by choosing an admissible mother [13] wavelet $\psi(\mathbf{x}) \in$ $L^{2}\left(\mathbb{R}^{2}\right)$, and from this function, a family of functions $\left\{\psi_{\boldsymbol{\xi}}(\mathbf{x})\right\}$ for $\boldsymbol{\xi}=[a, \theta, \mathbf{b}]^{T}$ is defined by:

$$
\psi_{\boldsymbol{\xi}}(\mathbf{x})=\mathcal{D}_{a} R_{\theta} \mathcal{T}_{\mathbf{b}}\{\psi\}(\mathbf{x})=U_{\boldsymbol{\xi}} \psi(\mathbf{x}) .
$$

$R_{\theta} g(\mathbf{x})=g\left(\boldsymbol{r}_{-\theta} x\right)$, is the rotation operator given in matrix notation as:

$$
\boldsymbol{r}_{\theta}=[\{\cos (\theta),-\sin (\theta)\},\{\sin (\theta), \cos (\theta)\}] .
$$

The purpose of including $\theta$ in the decomposition is to identify local behaviour like unidirectional, or separable structures, not aligned with the observational axes by rotating the analysis function over the full set of possible orientation, when necessary [13]. The wavelet coefficients of function $g(\mathbf{x})$ are then defined by [13]:

$$
w_{\psi}(\boldsymbol{\xi}, g)=\left\langle\psi_{\boldsymbol{\xi}}(\mathbf{x}), g(\mathbf{x})\right\rangle=\int_{-\infty}^{\infty} \int_{-\infty}^{\infty} g(\mathbf{x}) \psi_{\boldsymbol{\xi}}^{*}(\mathbf{x}) d^{2} \mathbf{x}
$$

where $*$ denotes the act of conjugation. Just like in 1-D the $w_{\psi}(\boldsymbol{\xi}, g)$ coefficients can be used to reconstruct the function $g(\mathbf{x})$ from a weighted average of the $\left\{\psi_{\boldsymbol{\xi}}(\mathbf{x})\right\}$ by:

$$
g(\mathbf{x})=\frac{1}{c_{\psi}} \int_{-\infty}^{\infty} \int_{-\infty}^{\infty} \int_{0}^{2 \pi} \int_{0}^{\infty} w_{\psi}(\boldsymbol{\xi}, g) \psi_{\boldsymbol{\xi}}(\mathbf{x}) \frac{d a d \theta d^{2} \mathbf{b}}{a^{3}} .
$$

To form $w_{\psi}(\boldsymbol{\xi}, g), g(\mathbf{x})$ has been localised in both space and spatial frequency depending on the form of $\psi(\mathbf{x})$. $\psi(\mathbf{x})$, the mother wavelet function, thus needs to be chosen with care, so that $w_{\psi}(\boldsymbol{\xi}, g)$ has suitable properties. We wish to derive $w_{\psi}(\boldsymbol{\xi}, g)$ that are locally 'analytic'.

Firstly we need to specify what we mean by 'analytic' in 2-D. A reasonable starting point to generalise $g^{(+)}\left(x_{1}\right)$ to 2-D is by considering 2-D versions of analytic functions and then forming the appropriate limit, as $\lim _{y_{1} \rightarrow 0^{+}} g^{(+)}\left(t_{1}\right)=g^{(+)}\left(x_{1}\right)$. The definition of a 2-D analytic function has previously been examined in detail in pure mathematics, within the field of Harmonic Analysis, by Stein and Weiss, see for example [37], [38]. Similar concepts have appeared in applied mathematics and geophysics [39], [40], and in signal processing [17], [25], [27]. An important fact to note is that the generalisation of analyticity to higher dimensions is not unique [38], and that more than a single generalisation of the Cauchy-Riemann system, and thus to the definition of a hyperanalytic function, is possible. Any function that satisfies a generalization of the Cauchy-Riemann system will in fact be referred to as a hyperanalytic function in this paper. A hyperanalytic signal is, in contrast to the hyperanalytic function, defined as the suitable limit of such a function.

Two possible hyperanalytic signals are relevant in this context: the hypercomplex signal of von Bülow and Sommer [17], and the monogenic signal of Felsberg and Sommer [25]. For clarity we will provide some discussion of the two hyperanalytic signals that are both possible and useful 2-D extensions of the 1-D analytic signal, corresponding in turn to four- and three-vector valued functions respectively. The hyperanalytic signal is usually represented as a quaternionic function, see for example [17], [25], i.e. as an object taking values in the skew field of quaternions, denoted $\mathbb{H}$. The advantage of using the quaternion representation is that convenient polar forms (magnitude and phase/s) are naturally introduced [17], [25], representing magnitude and variation of either separable or unidirectional variation. Once the choice of 'analytic' has been specified, or convenient local polar representation, the 'analytic' mother wavelet is formed. We approach the choice of mother wavelet by striving to separate disparate structures present at the same spatial position and to represent the isolated local structure, in terms of local structure (phase) and magnitude. The interpretation of the phase functions of these coefficients must be established, and this will depend on the form of $\psi(\mathbf{x})$, and assumptions regarding the local structure of $g(\mathbf{x})$. We shall construct quaternionic wavelets starting from a real 2-D wavelet that will be augmented into a quaternionic object, in analogue to 1-D analytic wavelets. The construction will rely on the choice of the starting point of a real mother wavelet, and the chosen form of hyperanalytic extension of this real function.

One starting point to the construction of the quaternionic mother wavelet is the anisotropic, or elongated, realvalued mother wavelet. Such a wavelet can construct coefficients representing plane wave or separable structures 
well. The wavelet would be extended into a quaternionic function so that a hyperanalytic wavelet coefficient representation can be formed of unidirectional or separable local structure in terms of the hyperanalytic phase/s and magnitude. Using quaternionic coefficients enables the easier parameterisation of local variation, and will rely on the transform coefficients being hyperanalytic, rather than the wavelet functions themselves. The strategy is related to work by Selesnick et al. [1] (who use directional complex wavelets), as well as Chan et al. [2] (who use separable quaternionic wavelets). Both the construction by Selesnick et al. and Chan et al. are implicitly based on the hypercomplex signal, and we explicitly construct a formal framework for interpreting such representations. We also form the monogenic extension of real directional wavelets.

Another possibility is to separate the signal into components present at given scales using an isotropic real wavelet, and then assuming locally at that spatial position and scale either plane waves, or potentially separable structures only are present, represent these by forming the hyperanalytic signal of the wavelet coefficients. This corresponds to the 2-D version of the wavelet projection representations proposed by Olhede and Walden [34].

One of the main questions to tackle in the construction is the appropriate interpretation that can be given to the quaternionic wavelet coefficients depending on the choice of mother wavelet function. The answer is complicated in 2-D by the fact that rotations do not commute with the operators that construct hyperanalytic images. Given the complication introduced by the rotations, we start by considering analysis when no such operation is required: we assume that the image is aligned with the observational axes, and set the angle of rotation in the decomposition equal to zero. We then construct wavelet functions such that the CWT coefficients are hyperanalytic signals in their spatial indexing, and refer to such mother wavelets as hyperanalyticizing. The mother wavelet function that constructs monogenic coefficients, is a monogenic signal, but we refer to any quaternionic analysis filter that produces hypercomplex wavelet coefficients as a hypercomplexing wavelet, thus explicitly noting that the wavelet produces hypercomplex coefficients but is not necessarily itself a hypercomplex signals. This then, when no rotation is implemented, produces hypercomplex wavelet coefficients from a real-valued image.

To deal with the act of rotation we introduce the definition of a $\theta$-hyperanalytic signal, that is a function if observed in some rotated frame of reference, is a hyperanalytic signal. Once the monogenic signal has been extended to $\theta$-monogenic, it follows that the quaternionic wavelet producing the $\theta$-monogenic wavelet coefficients is itself $\theta$-monogenic. In contrast, the hypercomplexing wavelet is not a $\theta$-hyperanalytic signal as the property does not even hold for $\theta=0$. We discuss in detail the interpretation of the wavelet coefficients constructed from the family of wavelet functions constructed by scaling, rotating and translating the hyperanalyticizing mother wavelet. In analogue to the 1-D analytic and anti-analytic decomposition of a real-valued signal, see [41], we introduce the decomposition of a real-valued image into $\theta$-hyperanalytic and anti-hyperanalytic components. The hyperanalyticizing wavelet annihilates/converts the anti-components to construct an object equal to the real CWT of a $\theta$-hyperanalytic function. This equivalence is important not only for the interpretation of the constructed coefficient but also allows for the derivation of additional useful properties of the hyperanalytic CWT.

Specific features of local structure may be extracted directly and easily from the defined wavelet coefficients, for instance potential local unidirectionality and separability in the rotated frame of reference may be characterised. For aggregations of oscillatory signals, the wavelets we develop in this paper, can be used to separate the multiple components, and simultaneously give simple descriptions of the components. Previous work on local analysis of oscillatory signals is clearly distinct, a number of authors, see for example [19], [21], [42], [43], have discussed wavelet ridge analysis using complex wavelets. The advantage of using quaternionic rather than complex wavelets, as suggested in this paper, is that separable oscillations admit a simple and compact representation. Furthermore for local plane waves using a suitable choice of quaternionic wavelets, it is not necessary to calculate the transform at all directions to determine the local plane wave description. Thus the usage of quaternionic wavelet functions either introduces the simplified description of additional texture structure, or simplifies the analysis procedure.

Coefficient equi- and invariance to local changes of phase and rotation alignment between the signal and the wavelet are also discussed. For the CWT this is not of immediate importance, as the transform coefficients exhibit equivariance with respect to changes in axes [13], but as discrete space decompositions may be related to continuous space decompositions, the stability of the decomposition to small spatial misalignment (phase-shifts) is very important [22]. For the hypercomplex and monogenic wavelet coefficients, we show that with a suitable choice of wavelet, magnitude invariance to phase shifts is achieved. For the monogenic wavelet coefficients, mother wavelet functions may be selected, such that the coefficients also exhibit invariance of magnitude with respect to local rotations. 
We discuss generic structures of mother wavelet constructions, i.e. combining separable/ isotropic/ directional scale localising procedures, with both the hypercomplex and monogenic signal representation, but also give specific families of mother wavelets falling into these classes. Existing quaternionic filters can be related to the hypercomplex and monogenic constructions [2], [44]-[47]. In analogue to 1-D wavelets approximating a local Fourier transform, most of the existing quaternionic wavelets have been constructed to serve the role of a local Quaternion Fourier Transform (QFT). In addition to [2], work in a similar vein is due to [48]-[51] and corresponds to constructing local versions of the QFT. He \& Yu [52], [53] constructed quaternionic multi-variate decompositions, and also work by Hsieh [54], [55] on motion smoothing should be noted, as well as work by Felsberg and Sommer on the monogenic scale space [47]. We provide a single complete framework that relates all of these constructions, and link the existing decompositions to Harmonic Analysis. We also construct completely new families of quaternionic wavelets that belong to the same family, and determine their properties.

Central to the developments in this paper is the concept of hyperanalyticity and local versions thereof. The hyperanalytic signal is developed as a generalization of the 1-D analytic signal. The analytic signal is best introduced via the 1-D Fourier transform, 1-D local oscillations and analytic functions, and so we start by a discussion of these aspects of the analytic signal.

\section{OSCILLATIONS, DIMENSIONALITY \& ANALYTICITY}

\section{A. Analyticity}

An oscillatory 1-D signal $c\left(x_{1}\right)$ is modelled as an amplitude and frequency modulated signal [29]:

$$
c\left(x_{1}\right)=a_{c}\left(x_{1}\right) \cos \left(2 \pi \varphi_{c}\left(x_{1}\right)\right)=\Re\left\{a_{c}\left(x_{1}\right) \exp \left(2 \pi \boldsymbol{j} \varphi_{c}\left(x_{1}\right)\right)\right\} .
$$

To determine the frequencies present in signal $c\left(x_{1}\right)$ the Fourier Transform (FT) of the signal could be calculated. The FT of a generic $d$-D signal $g(\mathbf{x})$, denoted $G(\mathbf{f})$, is defined by:

$$
G(\mathbf{f})=\int_{\mathbb{R}^{d}} g(\mathbf{x}) \exp \left(2 \boldsymbol{j} \pi \mathbf{f}^{T} \mathbf{x}\right) d^{d} \mathbf{x}, \quad G(\mathbf{f})=|G(\mathbf{f})| \exp \left(-2 \boldsymbol{j} \pi \varphi_{G}(\mathbf{f})\right),
$$

and $g(\mathbf{x})$ can be reconstructed via

$$
g(\mathbf{x})=\int_{\mathbb{R}^{d}}|G(\mathbf{f})| \exp \left(2 \boldsymbol{j} \pi\left(\mathbf{f}^{T} \mathbf{x}-\varphi_{G}(\mathbf{f})\right)\right) d^{d} \mathbf{f} .
$$

In 1-D at $x=x_{1}$ if $g\left(x_{1}\right)=c\left(x_{1}\right)$ is oscillatory, and has a clearly defined content at frequency $f_{1}=f_{0}\left(x_{1}\right)$ approximately we then find $c\left(x_{1}\right)=\left|C\left(f_{0}\left(x_{1}\right)\right)\right| \cos \left(2 \pi\left(f_{0}\left(x_{1}\right) x_{1}-\varphi_{C}\left(f_{0}\left(x_{1}\right)\right)\right)\right)$. To calculate the amplitude and phase of a real-valued signal, or $\left|C\left(f_{0}\left(x_{1}\right)\right)\right|$ and $2 \pi\left(f_{0}\left(x_{1}\right) x_{1}-\varphi_{C}\left(f_{0}\right)\right)$, from $c\left(x_{1}\right)$, the analytic extension of a signal could be used [23]. This is defined for an arbitrary real signal $g\left(x_{1}\right)$ by:

$$
g^{(+)}\left(x_{1}\right)=2 \int_{0}^{\infty} G(f) \exp \left(2 \pi \boldsymbol{j} f x_{1}\right) d f=g\left(x_{1}\right)+\boldsymbol{j} g^{(1)}\left(x_{1}\right) .
$$

$g^{(1)}\left(x_{1}\right)$ is the Hilbert Transform (HT) of $g\left(x_{1}\right)$. The HT in the time domain and the analytic signal in the frequency domain respectively, are

$$
g^{(1)}\left(x_{1}\right)=\mathcal{H}\{g\}\left(x_{1}\right)=\frac{1}{\pi} f_{-\infty}^{\infty} \frac{g(y)}{x_{1}-y} d y, \quad G^{(+)}\left(f_{1}\right)=G\left(f_{1}\right)\left(1+\operatorname{sgn}\left(f_{1}\right)\right) .
$$

The HT is as usual defined as a Cauchy principal value integral [23]. Using a stationary phase approximation [29] the analytic signal of $c\left(x_{1}\right)$ is then approximately $c^{(+)}\left(x_{1}\right)=c\left(x_{1}\right)+\boldsymbol{j} c^{(1)}\left(x_{1}\right)=\left|C\left(f_{0}\left(x_{1}\right)\right)\right| \exp \left(2 \boldsymbol{j} \pi\left(f_{0}\left(x_{1}\right) x_{1}-\right.\right.$ $\left.\left.\varphi_{C}\left(f_{0}\left(x_{1}\right)\right)\right)\right)+o(1)$. Clearly the amplitude and phase of $c\left(x_{1}\right)$ are retrieved by $a_{c}\left(x_{1}\right)=\left|c^{(+)}\left(x_{1}\right)\right|$, and $\varphi_{c}\left(x_{1}\right)=$ $\frac{1}{2 \pi} \tan ^{-1}\left(c^{(1)}\left(x_{1}\right) / c\left(x_{1}\right)\right)$. For a generic signal $g\left(x_{1}\right)$ the local amplitude and phase may be defined by:

$$
a_{g}\left(x_{1}\right)=\left|g^{(+)}\left(x_{1}\right)\right|, \quad \varphi_{g}\left(x_{1}\right)=\frac{1}{2 \pi} \tan ^{-1}\left(\frac{g^{(1)}\left(x_{1}\right)}{g\left(x_{1}\right)}\right) .
$$

For $a_{g}\left(x_{1}\right)$ and $\varphi_{g}\left(x_{1}\right)$ at point $x_{1}$ to be meaningful representations of $g\left(x_{1}\right)$, the latter signal is assumed to be at $x_{1}$ mainly limited in frequency to oscillations with a single period The key ingredient in defining a local amplitude and phase via equation (8) is the calculation of the analytic signal and the definition of $g^{(1)}\left(x_{1}\right)$. 
The 1-D analytic signal is the limit of an analytic function, where a function is denoted analytic when it satisfies the Cauchy-Riemann equations in the upper half of the complex plane. To construct an analytic function in a complex argument from a real function in a real argument, the Poisson kernels and Poisson convolutions are introduced [38]. The Poisson kernels, and the convolutions of $g(\cdot)$ with these kernels, are using an auxiliary variable $y$ defined by:

$$
p_{H}\left(t_{1}\right)=\frac{1}{\pi} \frac{y}{x_{1}^{2}+y^{2}}, \quad q_{H}\left(t_{1}\right)=\frac{1}{\pi} \frac{x_{1}}{x_{1}^{2}+y^{2}}, \quad u_{g}\left(t_{1}\right)=g(\cdot) * p_{H}(\cdot, y), \quad v_{g}^{(1)}\left(t_{1}\right)=g(\cdot) * q_{H}(\cdot, y) .
$$

Define complex-valued variable $t_{1}=x_{1}+\boldsymbol{j} y$, and note as $y \rightarrow 0^{+}, u_{g}\left(t_{1}\right) \rightarrow g\left(x_{1}\right)$ and $v_{g}^{(1)}\left(t_{1}\right) \rightarrow \mathcal{H}\{g\}\left(x_{1}\right)$, see [38] for details. $k_{g}^{( \pm)}\left(t_{1}\right)=u_{g}\left(t_{1}\right) \pm \boldsymbol{j} v_{g}^{(1)}\left(t_{1}\right)$ satisfies the Cauchy-Riemann equations in $\pm y_{1}>0$ [38], and are denoted analytic (+) and anti-analytic (-) functions, respectively. As $y \rightarrow 0^{ \pm} k_{g}^{( \pm)}\left(t_{1}\right) \rightarrow g^{( \pm)}\left(x_{1}\right)=$ $g\left(x_{1}\right) \pm \boldsymbol{j} \mathcal{H} g\left(x_{1}\right)$, and the limit of an analytic function is an analytic signal. Note that $g^{(+)}\left(t_{1}\right)$, i.e. the analytic signal evaluated at the complex argument, is still an analytic function for $y_{1}>0$, see also [23][p. 5]. More importantly the analytic and anti-analytic signals are represented in polar form by

$$
g^{( \pm)}\left(x_{1}\right)=\left|g^{( \pm)}\left(x_{1}\right)\right| e^{ \pm 2 \pi \boldsymbol{j} \phi_{g}\left(x_{1}\right)} .
$$

Any real signal $g\left(x_{1}\right) \in L^{2}(\mathbb{R})$ can be decomposed into an analytic and an anti-analytic signal [41]:

$$
\begin{aligned}
g\left(x_{1}\right) & =\frac{1}{2}\left[g^{(+)}\left(x_{1}\right)+g^{(-)}\left(x_{1}\right)\right]=\frac{1}{2}\left[g\left(x_{1}\right)+\boldsymbol{j} g^{(1)}\left(x_{1}\right)+g\left(x_{1}\right)-\boldsymbol{j} g^{(1)}\left(x_{1}\right)\right] \\
& =\left|a_{g}\left(x_{1}\right)\right| \cos \left(2 \pi \phi_{g}\left(x_{1}\right)\right),
\end{aligned}
$$

and so a local magnitude $a_{g}\left(x_{1}\right)$ and phase $\phi_{g}\left(x_{1}\right)$ can for any real-valued function $g\left(x_{1}\right)$ be defined from the modulus and phase of $g^{( \pm)}\left(x_{1}\right)$, whose forms are given by equation (10). We interpret equation (11) as the analytic and anti-analytic decompositions of $g\left(x_{1}\right)$, and this decomposition is important to derive the properties of the 1-D analytic wavelet transform, as demonstrated in [56][p. 426]. These well-known properties of the analytic signal need to be suitably extended to the multi-dimensional choice of 'analytic'.

\section{B. Hyperanalyticity}

In higher dimensions to determine equivalents of $a_{g}\left(x_{1}\right)$ and $\phi_{g}\left(x_{1}\right)$ from an observed real-valued function $g(\mathbf{x})$ the equivalent/s of function $g^{(1)}\left(x_{1}\right)$ must be defined and calculated. Based on these definitions the equivalents of $a_{g}\left(x_{1}\right)$ and $\phi_{g}\left(x_{1}\right)$ will then be given some suitable interpretation. For this purpose d-D extensions of the CauchyRiemann equations are used, and any given extension corresponding to a set of equations will be denoted a set of Generalized Cauchy-Riemann equations. In 1-D the spatial variable is 1-D, and there is a single associated auxiliary variable $y_{1}$, where an analytic function satisfies the Cauchy-Riemann equations in $y_{1}>0$. We consider, in 2-D and higher dimensions, augmenting the spatial variable $\mathbf{x}$ by a set of auxiliary variables collected in vector-valued variable $\mathbf{y}$, of dimension $p$ (some restrictions apply to the choice of $\mathbf{y}$, and its dimensionality), where $p$ will be related to the local structure of the image to be represented. We define $\Gamma^{(p)}=\left\{\mathbf{y}, y_{i}>0, i=1, \ldots, p\right\}$, and consider hyperanalyticity for $\mathbf{y} \in \Gamma^{(p)}$, and $\mathbf{x} \in \mathbb{R}^{d}$. Examples of such spaces include $p=1$ with $y_{1}>0$ and $\mathbf{x} \in \mathbb{R}^{d}$, as well as $\mathbf{x}$ and $\mathbf{y}$ of the same dimension, and $\mathbf{t}=\mathbf{x}+\boldsymbol{j} \mathbf{y}$ restricted to the cross-product of $d$ Euclidean upper half-planes, usually referred to as a tube see [38][p. 90], denoted $T_{\Gamma}^{(d)}$.

We shall be quite careful to distinguish between hyperanalytic functions, and their limits as $\mathbf{y} \rightarrow \mathbf{0}^{+}$. For future reference we make the following set of definition:

Definition 2.1: The hyperanalytic function.

Any vector-valued function in spatial variable $\mathbf{x}$, with associated $p$ dimensional auxiliary variable $\mathbf{y}$, that satisfies a given d-D generalisation of the Cauchy-Riemann equations for $\mathbf{y} \in \Gamma^{(p)}$ is denoted a hyperanalytic function. In analogue to the 1-D analytic signal, we define the hyperanalytic signal.

Definition 2.2: The hyperanalytic signal.

Any vector-valued function denoted $g^{(+\cdots+)}(\mathbf{x})$, that can be written as the limit as $\mathbf{y} \rightarrow \mathbf{0}^{+}$of a hyperanalytic function $k_{g}^{(+\cdots+)}(\mathbf{x}, \mathbf{y})$, with $p$-dimensional auxiliary variable $\mathbf{y}$ is denoted a hyperanalytic signal.

$g^{(+\cdots+)}(\mathbf{x})$ will have $p$ number of + 's to denote the dimensionality of the auxiliary variable $\mathbf{y}$. In 1-D we found that evaluating $g^{(+)}\left(x_{1}\right)$ at $x_{1}$ taking a complex value $t_{1}$, the analytic signal still corresponded to an analytic function in either the upper half plane. This will not in general be the case in higher dimensions and it will not be natural 
to represent the spatial variable as a complex-valued quantity: furthermore in some instances the dimension of $\mathbf{x}$ and $\mathbf{y}$ will not be the same. For future reference, to treat the rotation of the CWT, we also define a hyperanalytic function and signal in a rotated frame of reference.

Definition 2.3: The $\theta$-hyperanalytic function.

Any vector-valued function in spatial variable $\mathbf{x}^{\prime}=\boldsymbol{r}_{-\theta} \mathbf{x}$, with associated $p$ dimensional auxiliary variables $\mathbf{y}$, that satisfies a given d-D generalisation of the Cauchy-Riemann equations in variable $\mathbf{x}^{\prime}$ when $\mathbf{y}$ is in $\Gamma^{(p)}(\theta)$ is denoted a $\theta$-hyperanalytic function.

The $\theta$-hyperanalytic function, once the limit $\mathbf{y} \rightarrow \mathbf{0}^{+}$is taken, yields a $\theta$-hyperanalytic signal. Note that the choice of $\mathbf{y}$ may depend on $\theta$. The suitable choice of hyperanalytic to represent local image phenomena depends on the local dimensionality of the analysed image. To be able to make a decision on a suitable local representation, the local dimensionality of a narrowband oscillatory signal deserves some further discussion.

\section{2-D Oscillations \& narrowband signals}

In 2-D, assuming oscillations associated with a given period are observed, the local dimensionality of the image may be of many different forms. Define $\mathbf{f}_{0}\left(\phi_{0}\right)=\left[\begin{array}{ll}f_{0} \cos \left(\phi_{0}\right) & f_{0} \sin \left(\phi_{0}\right)\end{array}\right]^{T}$, and note that, if components are only present with period $1 / f_{0}$, this would imply the signal takes the form:

$$
g(\mathbf{x})=\int_{0}^{2 \pi} a_{g}\left(\phi_{0}\right) \cos \left(2 \pi \mathbf{x}^{T} \mathbf{f}_{0}\left(\phi_{0}\right)\right) d \phi_{0} .
$$

Thus the general representation of the image corresponds to a continuum of directions, and $a_{g}\left(\phi_{0}\right)$ is non-zero for many $\phi_{0} \in[0,2 \pi)$. Often locally the variation of the image is far more structured than the general form of equation (12) and admits a much simplified representation. For example, typical patterns such as plane waves would correspond to a single direction in the signal, or with a slight generalization of slow spatial modulation i.e.:

$$
g(\mathbf{x})=a_{g}\left(\phi_{0}(\mathbf{x}), \mathbf{x}\right) \cos \left(2 \pi \mathbf{x}^{T} \mathbf{f}_{0}\left(\phi_{0}(\mathbf{x}), \mathbf{x}\right)\right),
$$

whilst textured features [57] often take the form of an aggregation of plane waves:

$$
g(\mathbf{x})=\sum_{l=1}^{L} a_{g}\left(\phi_{0}^{(l)}(\mathbf{x}), \mathbf{x}\right) \cos \left(2 \pi \mathbf{x}^{T} \mathbf{f}_{0}\left(\phi_{0}^{(l)}(\mathbf{x}), \mathbf{x}\right)\right) .
$$

Not infrequently we find that $L=2$, and if this is the case then with $a_{g}\left(\phi_{0}^{(2)}(\mathbf{x}), \mathbf{x}\right)=a_{g}\left(\phi_{0}^{(1)}(\mathbf{x}), \mathbf{x}\right)$ and $\phi_{0}^{(2)}(\mathbf{x})=\phi_{0}^{(2)}(\mathbf{x})+\pi / 2$ and writing $\mathbf{x}=x\left[\begin{array}{ll}\cos (\chi) & \sin (\chi)\end{array}\right]^{T}$, we obtain

$$
g(\mathbf{x})=2 a_{g}\left(\phi_{0}^{(1)}(\mathbf{x}), \mathbf{x}\right) \cos \left(f_{0} x \cos \left(\chi+\phi_{0}^{(1)}(\mathbf{x})\right)\right) \cos \left(f_{0}(\mathbf{x}) x \sin \left(\chi+\phi_{0}^{(1)}\right)\right),
$$

and the signal is separable. Key local characterising features of the image will correspond to $L$, the local dimensionality of the oscillation at spatial point $\mathbf{x}, f_{0}(\mathbf{x})$, the local period, and the form of the $L$ pairs $\left\{a_{g}\left(\phi_{0}^{(l)}(\mathbf{x}), \mathbf{x}\right), \phi^{(l)}(\mathbf{x})\right\}$, that is the magnitude and direction of the $l$ th component. We consider three special forms of local structure: the local plane wave, that is $L=1$, local separable behaviour, that is $L=2$ and $\phi_{0}^{(2)}(\mathbf{x})=\phi_{0}^{(1)}(\mathbf{x})+\pi / 2$, and $L>1$ with no special relationship between $\phi_{0}^{(2)}(\mathbf{x})$ and $\phi_{0}^{(1)}(\mathbf{x})$. In the latter case there is no local separable structure present.

The analysis problem then corresponds to, if confronted with a single real image that naturally fits into the model of equation (13), or for that matter equation (14), determining $a_{g}^{(l)}\left(\phi_{0}(\mathbf{x}), \mathbf{x}\right), f_{0}(\mathbf{x})$, and $\phi_{0}^{(l)}(\mathbf{x})$ from the single observed image, or equivalently defining the 2-D set of functions playing the role of $g^{(+)}\left(x_{1}\right)$, from $g(\mathbf{x})$. Once this has been achieved, the characterising parameters may be determined from $g^{(+)}(\mathbf{x})$. In analogue with 1-D, a natural starting point for such determination, is a sinusoidal decomposition. 


\section{Quaternionic Fourier Transforms}

The FT decomposes structure into plane waves of $\exp \left(2 \pi \boldsymbol{j} \mathbf{f}^{T} \mathbf{x}\right),[18]$ and does not represent separable structures as a single coefficient. A natural tool for analysis of separable oscillations is instead the Quaternionic Fourier Transform (QFT) [18]. Instead of using complex numbers, the QFT is defined in terms of quaternionic units [58], [59]. An arbitrary quaternionic object takes the form $q=q_{1}+q_{2} \boldsymbol{i}+q_{3} \boldsymbol{j}+q_{4} \boldsymbol{k} \in \mathbb{H}$ where $q_{i} \in \mathbb{R}, i=1, \ldots 4$ and $\boldsymbol{i}^{2}=\boldsymbol{j}^{2}=\boldsymbol{k}^{2}=\boldsymbol{i} \boldsymbol{j} \boldsymbol{k}=-1$, whilst $\boldsymbol{i} \boldsymbol{j}=-\boldsymbol{j} \boldsymbol{i}=\boldsymbol{k}, \boldsymbol{i} \boldsymbol{k}=-\boldsymbol{k} \boldsymbol{i}=-\boldsymbol{j}$, and finally $\boldsymbol{j} \boldsymbol{k}=-\boldsymbol{k} \boldsymbol{j}=\boldsymbol{i}$. $q$ has conjugate $q^{*}=q_{1}-q_{2} \boldsymbol{i}-q_{3} \boldsymbol{j}-q_{4} \boldsymbol{k}$, and $q$ can be written as $q=\Re\{q\}+\mathscr{P} u\{q\}$, where $\Re\{q\}=\frac{1}{2}\left[q+q^{*}\right]$, is the real part and $\mathscr{P} u\{q\}=\frac{1}{2}\left[q-q^{*}\right]$, is the pure quaternion part. The QFT is then defined in 2-D by [60]:

$$
G_{Q}(\mathbf{q})=\mathcal{F}_{\mathcal{Q}}\{g\}(\mathbf{q})=\int_{\mathbb{R}^{2}} \exp \left(-2 \pi \boldsymbol{i} x_{1} q_{1}\right) g(\mathbf{x}) \exp \left(-2 \pi \boldsymbol{j} x_{2} q_{2}\right) d^{2} \mathbf{x},
$$

and inverted by

$$
g(\mathbf{x})=\mathcal{F}_{\mathcal{Q}}^{-1}\left\{G_{Q}\right\}(\mathbf{x})=\int_{\mathbb{R}^{2}} \exp \left(2 \pi \boldsymbol{i} x_{1} q_{1}\right) G_{Q}(\mathbf{q}) \exp \left(2 \pi \boldsymbol{j} x_{2} q_{2}\right) d^{2} \mathbf{x} .
$$

The QFT can recover separate parity structure in $x_{1}$ and $x_{2}$ as it separately records four real values at each quaternion frequency $\mathbf{q}$, thus forming a natural analysis tool for structure separable over $x_{1}$ and $x_{2}$ in terms of $\cos \left(2 \pi x_{1} q_{1}\right) \cos \left(2 \pi x_{2} q_{2}\right), \cos \left(2 \pi x_{1} q_{1}\right) \sin \left(2 \pi x_{2} q_{2}\right), \sin \left(2 \pi x_{1} q_{1}\right) \cos \left(2 \pi x_{2} q_{2}\right)$, and $\sin \left(2 \pi x_{1} q_{1}\right) \sin \left(2 \pi x_{2} q_{2}\right)$, each corresponding to a separable function in $x_{1}$ and $x_{2}$, that is either even or odd. A local representation of this form would require defining more than a single additional component $g^{(1)}(\mathbf{x})$ at $\mathbf{x}$ as both local rates corresponding to $q_{1}$ and $q_{2}$ would need determination.

The Fourier transform of a signal $g(\mathbf{x})$ can also be defined in an arbitrary pure unit quaternion $\boldsymbol{e}=\boldsymbol{i} e_{1}+\boldsymbol{j} e_{2}+\boldsymbol{k} e_{3}$, where $\sum e_{j}^{2}=1$, can also be calculated, and is given by:

$$
G_{\boldsymbol{e}}(\mathbf{f})=\int_{-\infty}^{\infty} \int_{-\infty}^{\infty} g(\mathbf{x}) e^{-2 \pi \boldsymbol{e} \mathbf{f}^{T} \mathbf{x}} d^{2} \mathbf{x}, \quad g(\mathbf{x})=\int_{-\infty}^{\infty} \int_{-\infty}^{\infty} G_{\boldsymbol{e}}(\mathbf{f}) e^{2 \pi \boldsymbol{e} \mathbf{f}^{T} \mathbf{x}} d^{2} \mathbf{f} .
$$

We will refer to the above transformation (18) as the Unit Quaternion Fourier Transform (UQFT), but it is also known as the Type 3 QFT, see [60][p. 2784]. The UQFT is still interpretable as a sinusoidal decomposition of structure, as any pure unit $e$ satisfies the De Moivre relationships [61]. The representation is now in terms of plane waves, i.e. $\cos \left(2 \pi \mathbf{f}^{T} \mathbf{x}\right)$ and $\sin \left(2 \pi \mathbf{f}^{T} \mathbf{x}\right)$, and would require more than a single component $g^{(1)}(\mathbf{x})$ as both the direction and period of $\mathbf{f}$ would require determination from the signal. For signals with simple local dimensionality, the QFT and the UQFT form natural representations of the signal. Using the FT allowed us to obtain the analytic signal/function representation of $c\left(x_{1}\right)$ via the stationary phase approximation and we shall discuss the hyperanalytic signals/functions naturally linked with the QFT and UQFT.

\section{E. The hypercomplex signal}

To extend the analytic signal to 2-D von Bülow and Sommer [17] used partial HTs [27] in both of the spatial directions, thus defining the hypercomplex signal. The partial HT of $g(\mathbf{x})$ in either of $x_{l}, l=1$, 2, when the second argument is fixed, is denoted $\mathcal{H}_{l}\{g\}(\mathbf{x}), l=1,2$ and the total Hilbert transform is formed by $\mathcal{H}_{T}\{g\}(\mathbf{x})=$ $\mathcal{H}_{2}\left\{\mathcal{H}_{1}\{g\}\right\}(\mathbf{x}) . \mathcal{H}_{T}\{g\}(\mathbf{x})$ corresponds to the consecutive operation of two partial HTs [27].

Definition 2.4: The hypercomplex signal.

The hypercomplex signal extension of an image $g(\mathbf{x})$ is defined by

$$
g^{(++)}(\mathbf{x})=\mathcal{H C} g(\mathbf{x})=g(\mathbf{x})+\boldsymbol{i} g_{H}^{(1)}(\mathbf{x})+\boldsymbol{j} g_{H}^{(2)}(\mathbf{x})+\boldsymbol{k} g_{H}^{(3)}(\mathbf{x}),
$$

where

$$
g_{H}^{(1)}(\mathbf{x})=\mathcal{H}_{1}\{g\}(\mathbf{x}), \quad g_{H}^{(2)}(\mathbf{x})=\mathcal{H}_{2}\{g\}(\mathbf{x}) \quad g_{H}^{(3)}(\mathbf{x})=\mathcal{H}_{T}\{g\}(\mathbf{x}) .
$$

To confirm that the hypercomplex signal corresponds to a valid hyperanalytic signal we firstly need to define a hyperanalytic function and then introduce two auxiliary variables, $y_{1}$ and $y_{2}$. We define the set of functions

$$
\begin{aligned}
& u_{g ; H}(\mathbf{x}, \mathbf{y})=p\left(x_{1}, y_{1}\right) * p\left(x_{2}, y_{2}\right) * g(\mathbf{x}), \quad v_{g ; H}^{(1)}(\mathbf{x}, \mathbf{y})=q_{1}\left(x_{1}, y_{1}\right) * p\left(x_{2}, y_{2}\right) * g(\mathbf{x}) \\
& v_{g ; H}^{(2)}(\mathbf{x}, \mathbf{y})=p\left(x_{1}, y_{1}\right) * q_{1}\left(x_{2}, y_{2}\right) * g(\mathbf{x}), \quad v_{g ; H}^{(3)}(\mathbf{x}, \mathbf{y})=q_{1}\left(x_{1}, y_{1}\right) * q_{1}\left(x_{2}, y_{2}\right) * g(\mathbf{x}),
\end{aligned}
$$


where $p(x, y)$ and $q_{1}(x, y)$ are given by equation (9). Collect the spatial and auxiliary variables in the vector-valued variable $\mathbf{t}=\mathbf{x}+\boldsymbol{j}\left(\begin{array}{ll}\boldsymbol{k} y_{1} & y_{2}\end{array}\right)^{T}$. Define the quaternionic (or vector-valued) function:

$$
k_{g ; H}^{(++)}(\mathbf{x}, \mathbf{y})=u_{g ; H}(\mathbf{x}, \mathbf{y})+\boldsymbol{i} v_{g ; H}^{(1)}(\mathbf{x}, \mathbf{y})+\boldsymbol{j} v_{g ; H}^{(2)}(\mathbf{x}, \mathbf{y})+\boldsymbol{k} v_{g ; H}^{(3)}(\mathbf{x}, \mathbf{y}) .
$$

It is convenient to express $k_{g ; H}^{(++)}(\mathbf{x}, \mathbf{y})$ in terms of its Cayley-Dickson forms [62][p. 1952]. We define $u_{g ; H}^{(i)}(\mathbf{x}, \mathbf{y})=$ $u_{g ; H}(\mathbf{x}, \mathbf{y})+\boldsymbol{j} v_{g ; H}^{(2)}(\mathbf{x}, \mathbf{y}), v_{g ; H}^{(i)}(\mathbf{x}, \mathbf{y})=v_{g ; H}^{(1)}(\mathbf{x}, \mathbf{y})+\boldsymbol{j} v_{g ; H}^{(3)}(\mathbf{x}, \mathbf{y}), u_{g ; H}^{(j)}(\mathbf{x}, \mathbf{y})=u_{g ; H}(\mathbf{x}, \mathbf{y})+\boldsymbol{j} v_{g ; H}^{(1)}(\mathbf{x}, \mathbf{y})$ and $v_{g ; H}^{(j)}(\mathbf{x}, \mathbf{y})=v_{g ; H}^{(2)}(\mathbf{x}, \mathbf{y})+\boldsymbol{j} v_{g ; H}^{(3)}(\mathbf{x}, \mathbf{y})$. The functions $u_{g ; H}^{(\cdot)}(\mathbf{x}, \mathbf{y})$ and $v_{g ; H}^{(\cdot)}(\mathbf{x}, \mathbf{y})$, with $\cdot=i, j$, are the simplex and perplex respectively, of the Cayley-Dickson forms. Thus it in fact follows that

$$
k_{g ; H}^{(++)}(\mathbf{x}, \mathbf{y})=u_{g ; H}^{(i)}(\mathbf{x}, \mathbf{y})+i v_{g ; H}^{(i)}(\mathbf{x}, \mathbf{y})=u_{g ; H}^{(j)}(\mathbf{x}, \mathbf{y})+\boldsymbol{j} v_{g ; H}^{(j)}(\mathbf{x}, \mathbf{y}) .
$$

$u_{g ; H}^{(i)}(\mathbf{t}), v_{g ; H}^{(i)}(\mathbf{t}) \in \mathbb{C}$ and the pair of functions satisfies the Cauchy-Riemann equations in $t_{1}$ for fixed values of $t_{2}$, in the upper half plane of $y_{1}>0$, whilst $u_{g ; H}^{(j)}(\mathbf{t}) \in \mathbb{C}, v_{g ; H}^{(j)}(\mathbf{t}) \in \mathbb{C}$, and the pair satisfies the Cauchy-Riemann equations in $t_{2}$ for fixed values of $t_{1}$, in the upper half plane of $y_{2}>0$. The hypercomplex system of equations is defined as:

$$
\begin{array}{ll}
\frac{\partial u_{g ; H}^{(i)}(\mathbf{t})}{\partial y_{1}}+\frac{\partial v_{g ; H}^{(i)}(\mathbf{t})}{\partial x_{1}}=0, & \frac{\partial u_{g ; H}^{(i)}(\mathbf{t})}{\partial x_{1}}-\frac{\partial v_{g ; H}^{(i)}(\mathbf{t})}{\partial y_{1}}=0 \\
\frac{\partial u_{g ; H}^{(j)}(\mathbf{t})}{\partial y_{2}}+\frac{\partial v_{g ; H}^{(j)}(\mathbf{t})}{\partial x_{2}}=0, & \frac{\partial u_{g ; H}^{(j)}(\mathbf{t})}{\partial x_{2}}-\frac{\partial v_{g ; H}^{(j)}(\mathbf{t})}{\partial y_{2}}=0 .
\end{array}
$$

This set of equations provide a valid 2-D generalisation of the Cauchy-Riemann equations, and thus, as the set of functions constituting $k_{g ; H}^{(++)}(\mathbf{x}, \mathbf{y})$ satisfy equations (22) for $(\mathbf{x}, \mathbf{y})$ taking values in the tube $T_{\Gamma}$, we may denote the latter function hyperanalytic, $c f$ definition (2.1). Clearly as $\mathbf{y} \rightarrow \mathbf{0}^{+}, u_{g ; H}(\mathbf{t}) \rightarrow g(\mathbf{x}), v_{g ; H}^{(1)}(\mathbf{t}) \rightarrow$ $g_{H}^{(1)}(\mathbf{x}), v_{g ; H}^{(2)}(\mathbf{t}) \rightarrow g_{H}^{(2)}(\mathbf{x})$, and $v_{g ; H}^{(3)}(\mathbf{t}) \rightarrow g_{H}^{(3)}(\mathbf{x})$, and thus $\lim _{\mathbf{y} \rightarrow \mathbf{0}^{+}} k_{g ; H}^{(++)}(\mathbf{x}, \mathbf{y}) \rightarrow g^{(++)}(\mathbf{x})$, and so $g^{(++)}(\mathbf{x})$ corresponds to a hyperanalytic signal ( $c f$ definition $(2.2)$.

Furthermore, the hypercomplex signal $g^{(++)}(\mathbf{x})$, when viewed in the quaternionic frequency domain [17][p. 2849], satisfies $G_{Q}^{(++)}(\mathbf{q})=0 \quad \forall \mathbf{q} \notin \mathbb{R}^{+2}$, this providing a 2-D version of equation (7).

To be able to consider separable structures in an arbitrary direction we extend the definition of a hypercomplex signal to a $\theta$-hypercomplex signal.

Definition 2.5: The $\theta$-hypercomplex signal.

The $\theta$-hypercomplex signal of a real-valued image $g(\mathbf{x})$ is defined for any $\theta \in[0,2 \pi)$, as

$$
\begin{aligned}
g_{\theta}^{(++)}(\mathbf{x}) & =\mathcal{H C} \mathcal{C}_{\theta} g(\mathbf{x})=g\left(\boldsymbol{r}_{-\theta} \mathbf{x}\right)+\boldsymbol{i} g_{H}^{(1)}\left(\boldsymbol{r}_{-\theta} \mathbf{x}\right)+\boldsymbol{j} g_{H}^{(2)}\left(\boldsymbol{r}_{-\theta} \mathbf{x}\right)+\boldsymbol{k} g_{H}^{(3)}\left(\boldsymbol{r}_{-\theta} \mathbf{x}\right) \\
& =R_{\theta} g(\mathbf{x})+\boldsymbol{i} R_{\theta} \mathcal{H}_{1} g(\mathbf{x})+\boldsymbol{j} R_{\theta} \mathcal{H}_{2} g(\mathbf{x})+\boldsymbol{k} R_{\theta} \mathcal{H}_{1} \mathcal{H}_{2} g(\mathbf{x}) \\
& =g_{\theta}(\mathbf{x})+\boldsymbol{i} g_{\theta ; H}^{(1)}(\mathbf{x})+\boldsymbol{j} g_{\theta ; H}^{(2)}(\mathbf{x})+\boldsymbol{k} g_{\theta ; H}^{(3)}(\mathbf{x}),
\end{aligned}
$$

thus defining the functions $g_{\theta ; H}^{(s)}(\mathbf{x})$, for $s=1,2,3$. The $\theta$-hypercomplex signal is suitable for analysing structure that is separable, or aligned with variations, in a rotated frame of reference. To be able to compactly note the QFT of $g_{\theta}^{(+)}(\mathbf{x})$ define the reflection operator $\mathcal{J}_{\theta}$. The reflection of $g(\mathbf{x})$ in the line that makes an angle $\theta$ with the $x_{1}$ axis is given by $\mathcal{J}_{\theta} g(\mathbf{x})=g\left(\boldsymbol{J}_{\theta} \mathbf{x}\right)$, using matrix $\boldsymbol{J}_{\theta}=[\{\cos (2 \theta), \sin (2 \theta)\},\{\sin (2 \theta),-\cos (2 \theta)\}]$, where $\theta \in[0,2 \pi)$. Then:

$$
\begin{aligned}
\mathcal{F}\left\{g_{\theta}^{(++)}\right\}(\mathbf{f})= & {\left[1+\operatorname{sgn}\left(\left[\boldsymbol{r}_{-\theta} \mathbf{f}\right]_{1}\right)\right]\left[1-\boldsymbol{k} \operatorname{sgn}\left(\left[\boldsymbol{r}_{-\theta} \mathbf{f}\right]_{2}\right)\right] G\left(\boldsymbol{r}_{-\theta} \mathbf{f}\right) } \\
\mathcal{F}_{\mathcal{Q}}\left\{g_{\theta}^{(++)}\right\}(\mathbf{q})= & \frac{1-\boldsymbol{k}}{2}\left[1+\operatorname{sgn}\left(\left[\boldsymbol{r}_{-\theta} \mathbf{q}\right]_{1}\right)\right]\left[1+\operatorname{sgn}\left(\left[\boldsymbol{r}_{-\theta} \mathbf{q}\right]_{2}\right)\right] G\left(\boldsymbol{r}_{-\theta} \mathbf{q}\right) \\
& +\frac{1+\boldsymbol{k}}{2}\left[1+\operatorname{sgn}\left(\left[\boldsymbol{r}_{\theta} \mathbf{q}\right]_{1}\right)\right]\left[1+\operatorname{sgn}\left(\left[\boldsymbol{r}_{\theta} \mathbf{q}\right]_{2}\right)\right] G\left(\boldsymbol{r}_{-\theta} \boldsymbol{J}_{\pi / 2} \mathbf{q}\right)
\end{aligned}
$$

\footnotetext{
${ }^{2}$ Formally $t_{1}=x_{1}+i y_{1}$, but any number system of the form of a real number plus a scaled pure unit quaternion is isomorphic to $\mathbb{C}$, and so we will relax this formality and not distinguish $\mathbb{C}=\{x+\boldsymbol{j} y\}$ from $\{x+\boldsymbol{i} y\}$.
} 
The result follows by Appendix [-A combined with the fact that the QFT of a real-valued signal $g(\mathbf{x})$ can be calculated from its Fourier transform (see Hahn \& Snopek [26] as well as Pei, Ding and Chang [60]). Thus the FT of $g_{\theta}^{(++)}(\mathbf{x})$ is supported on $\left[\boldsymbol{r}_{-\theta} \mathbf{f}\right]_{2}>0$, whilst as noted in the appendix, the QFT if implemented in a rotated frame is supported wholly on one quadrant. Most importantly, as the QFT can be inverted into the spatial domain signal, if a signal admits a QFT representation of equation A-1 then it is the QFT of a $\theta$-hypercomplex signal identified by the form of $G\left(\boldsymbol{r}_{-\theta} \mathbf{q}\right)$. This result can be used to interpret the properties of the quaternionic wavelet coefficients.

We shall define analysis methods suitable for signals observed in an alternate frame of reference, as the local orientation of a signal is a very important local characteristic. Define the signal $\widetilde{g}_{-\theta}(\mathbf{x})$ via

$$
\widetilde{g}_{-\theta}(\mathbf{x})=g\left(\boldsymbol{r}_{\theta} \mathbf{x}\right), \quad g(\mathbf{x})=\widetilde{g}_{-\theta}\left(\boldsymbol{r}_{-\theta} \mathbf{x}\right), \theta \in[0,2 \pi),
$$

so that $\widetilde{g}_{-\theta ; \theta}^{(++)}(\mathbf{x})=\widetilde{g}_{-\theta}\left(\boldsymbol{r}_{-\theta} \mathbf{x}\right)+i \widetilde{g}_{-\theta ; H}^{(1)}\left(\boldsymbol{r}_{-\theta} \mathbf{x}\right)+\boldsymbol{j} \widetilde{g}_{-\theta ; H}^{(2)}\left(\boldsymbol{r}_{-\theta} \mathbf{x}\right)+\boldsymbol{k} g_{-\theta ; H}^{(3)}\left(\boldsymbol{r}_{-\theta} \mathbf{x}\right)$, or $\widetilde{g}_{-\theta ; \theta}^{(++)}(\mathbf{x})=g(\mathbf{x})+\boldsymbol{i} \widetilde{g}_{-\theta ; H}^{(1)}\left(\boldsymbol{r}_{-\theta} \mathbf{x}\right)$ $+\boldsymbol{j} \widetilde{g}_{-\theta ; H}^{(2)}\left(\boldsymbol{r}_{-\theta} \mathbf{x}\right)+\boldsymbol{k} g_{-\theta ; H}^{(3)}\left(\boldsymbol{r}_{-\theta} \mathbf{x}\right)$. Of course, $\widetilde{g}_{-\theta}\left(\boldsymbol{r}_{-\theta} \mathbf{x}\right)=R_{\theta} R_{-\theta} g(\mathbf{x})=g(\mathbf{x})$, but $\widetilde{g}_{-\theta, \theta ; H}^{(s)}=\widetilde{g}_{-\theta ; H}^{(s)}\left(\boldsymbol{r}_{-\theta} \mathbf{x}\right)=$ $R_{\theta} \mathcal{H}_{s} R_{-\theta} g(\mathbf{x}) \neq g_{H}^{(s)}(\mathbf{x}), s=1,2$, etc. Hence $\widetilde{g}_{-\theta}\left(\boldsymbol{r}_{-\theta} \mathbf{x}\right)$ corresponds to the observed signal, whilst $\widetilde{g}_{-\theta, \theta ; H}^{(s)}$ corresponds to the HTs in a rotated frame of reference observed in our frame of reference.

Definition 2.6: $\theta$-hypercomplex: hypercomplex vs anti-hypercomplex decomposition.

The decomposition of real-valued $g(\mathbf{x})$ into four quaternionic components $\widetilde{g}_{-\theta ; \theta}^{(++)}(\mathbf{x}), \widetilde{g}_{-\theta ; \theta}^{(-+)}(\mathbf{x}), \widetilde{g}_{-\theta ; \theta}^{(+-)}(\mathbf{x})$, and $\widetilde{g}_{-\theta ; \theta}^{(--)}(\mathbf{x}):$

$$
g(\mathbf{x})=\frac{1}{4}\left(\widetilde{g}_{-\theta ; \theta}^{(++)}(\mathbf{x})+\widetilde{g}_{-\theta ; \theta}^{(-+)}(\mathbf{x})+\widetilde{g}_{-\theta ; \theta}^{(+-)}(\mathbf{x})+\widetilde{g}_{-\theta ; \theta}^{(--)}(\mathbf{x})\right),
$$

where we define $\widetilde{g}_{-\theta ; \theta}^{(++)}(\mathbf{x})=g(\mathbf{x})+i \widetilde{g}_{-\theta ; H}^{(1)}\left(\boldsymbol{r}_{-\theta} \mathbf{x}\right)+\boldsymbol{j} \widetilde{g}_{-\theta ; H}^{(2)}\left(\boldsymbol{r}_{-\theta} \mathbf{x}\right)+\boldsymbol{k} g_{-\theta ; H}^{(3)}\left(\boldsymbol{r}_{-\theta} \mathbf{x}\right), \widetilde{g}_{-\theta ; \theta}^{(-+)}(\mathbf{x})=g(\mathbf{x})-$ $\boldsymbol{i} \widetilde{g}_{-\theta ; H}^{(1)}\left(\boldsymbol{r}_{-\theta} \mathbf{x}\right)+\boldsymbol{j} \widetilde{g}_{-\theta ; H}^{(2)}\left(\boldsymbol{r}_{-\theta} \mathbf{x}\right)-\boldsymbol{k} g_{-\theta ; H}^{(3)}\left(\boldsymbol{r}_{-\theta} \mathbf{x}\right), \widetilde{g}_{-\theta ; \theta}^{(+-)}(\mathbf{x})=g(\mathbf{x})+\boldsymbol{i} \widetilde{g}_{-\theta ; H}^{(1)}\left(\boldsymbol{r}_{-\theta} \mathbf{x}\right)-\boldsymbol{j} \widetilde{g}_{-\theta ; H}^{(2)}\left(\boldsymbol{r}_{-\theta} \mathbf{x}\right)-\boldsymbol{k} g_{-\theta ; H}^{(3)}\left(\boldsymbol{r}_{-\theta} \mathbf{x}\right)$ and $\widetilde{g}_{-\theta ; \theta}^{(--)}(\mathbf{x})=g(\mathbf{x})-i \widetilde{g}_{-\theta ; H}^{(1)}\left(\boldsymbol{r}_{-\theta} \mathbf{x}\right)-\boldsymbol{j} \widetilde{g}_{-\theta ; H}^{(2)}\left(\boldsymbol{r}_{-\theta} \mathbf{x}\right)+\boldsymbol{k} g_{-\theta ; H}^{(3)}\left(\boldsymbol{r}_{-\theta} \mathbf{x}\right)$. will be denoted as the $\theta$-Hypercomplex analytic/anti-analytic decomposition of $g(\mathbf{x})$. The four components $\widetilde{g}_{-\theta ; \theta}^{(++)}(\mathbf{x}), \widetilde{g}_{-\theta ; \theta}^{(-+)}(\mathbf{x}), \widetilde{g}_{-\theta ; \theta}^{(+-)}(\mathbf{x})$, and $\widetilde{g}_{-\theta ; \theta}^{(--)}(\mathbf{x})$, are denoted as the $\theta$-hypercomplex signal, the $\theta$ first anti-hypercomplex signal, the $\theta$ second anti-hypercomplex signal and the $\theta$ third anti-hypercomplex signal, respectively.

In the rotated frame of reference, equation (27) provides a 2-D analogue of the analytic/anti-analytic decomposition of a 1-D real-valued signal [41]. We may use the $\theta$-hypercomplex signal to decompose any real image $g(\mathbf{x})$ into four components, where each component in a rotated frame of reference is analytic/anti-analytic in each spatial variable separately. We characterize local structure in terms of magnitude and phase of $\widetilde{g}_{-\theta ; \theta}^{(++)}(\mathbf{x})$, interpretable in the rotated frame of reference. Any of $\widetilde{g}_{-\theta, \theta}^{( \pm \pm)}(\mathbf{x})$ if calculated at all $\theta=[0, \pi / 2]$, will enable us to parameterise separable oscillations. The $\theta$-hypercomplex signal is introduced in this article for the purpose of recognizing separable or univariate local structure that is not aligned with the axis of observation; the act of rotations is integral to defining the CWT in subsequent sections, and so its introduction is a necessity.

\section{F. Properties of the hypercomplex signal}

A hypercomplex signal can be decomposed in terms of a modulus and a set of phase/s, using quaternionic polar coordinates, see discussion by [63]. The modulus is still a real number and can be interpreted as local energy content whereas the phase/s are given by a unit energy quaternion and contain additional information about the structure of the image. This, extended to the $\theta$-hypercomplex signal, corresponds to a polar representation of variation in orientation $\theta$.

Definition 2.7: The polar representation of the $\theta$-hypercomplex signal.

The $\theta$-hypercomplex image will be represented in terms of its amplitude, and phases. Define the unit quaternion by $q_{\theta}^{(U)}(\mathbf{x})=q_{\theta}^{(1)}(\mathbf{x})+\boldsymbol{i} q_{\theta}^{(2)}(\mathbf{x})+\boldsymbol{j} q_{\theta}^{(3)}(\mathbf{x})+\boldsymbol{k} q_{\theta}^{(4)}(\mathbf{x})=e^{2 \pi \boldsymbol{i} \alpha_{\theta}(\mathbf{x})} e^{2 \pi \boldsymbol{k} \gamma_{\theta}(\mathbf{x})} e^{2 \pi \boldsymbol{j} \beta_{\theta}(\mathbf{x})}$, then the polar representation corresponds to:

$$
g_{\theta}^{(++)}(\mathbf{x})=\left|g_{\theta}^{(++)}(\mathbf{x})\right| e^{2 \pi \boldsymbol{i} \alpha_{\theta}(\mathbf{x})} e^{2 \pi \boldsymbol{k} \gamma_{\theta}(\mathbf{x})} e^{2 \pi \boldsymbol{j} \beta_{\theta}(\mathbf{x})}=\left|g_{\theta}^{(++)}(\mathbf{x})\right| q_{\theta}^{(U)}(\mathbf{x})
$$


Von Bülow and Sommer considered only the hypercomplex signal (i.e. $\theta=0$ ) but the exact algebraic forms of the angles for both $\alpha_{\theta}(\mathbf{x})$ and $\beta_{\theta}(\mathbf{x})$ in terms of $g_{\theta, H}^{(l)}(\mathbf{x}), l=1,2,3$, can be found by substituting in the form for the quaternion components into the formulae given by [17][p. 2849]:

$$
\begin{aligned}
& \alpha_{\theta}(\mathbf{x})=\frac{1}{4 \pi} \tan ^{-1}\left(2 \frac{q_{\theta}^{(3)}(\mathbf{x}) q_{\theta}^{(4)}(\mathbf{x})+q_{\theta}^{(1)}(\mathbf{x}) q_{\theta}^{(2)}(\mathbf{x})}{q_{\theta}^{(1) 2}(\mathbf{x})+q_{\theta}^{(3) 2}(\mathbf{x})-\left(q_{\theta}^{(2) 2}(\mathbf{x})+q_{\theta}^{(4) 2}(\mathbf{x})\right)}\right), \\
& \beta_{\theta}(\mathbf{x})=\frac{1}{4 \pi} \tan ^{-1}\left(2 \frac{q_{\theta}^{(2)}(\mathbf{x}) q_{\theta}^{(4)}(\mathbf{x})+q_{\theta}^{(1)}(\mathbf{x}) q_{\theta}^{(3)}(\mathbf{x})}{q_{\theta}^{(1) 2}(\mathbf{x})+q_{\theta}^{(2) 2}(\mathbf{x})-\left(q_{\theta}^{(3) 2}(\mathbf{x})+q_{\theta}^{(4) 2}(\mathbf{x})\right)}\right), \\
& \gamma_{\theta}(\mathbf{x})=-\frac{1}{4 \pi} \sin ^{-1}\left(2\left[q_{\theta}^{(2)}(\mathbf{x}) q_{\theta}^{(3)}(\mathbf{x})-q_{\theta}^{(1)}(\mathbf{x}) q^{(4)}(\mathbf{x})\right]\right) .
\end{aligned}
$$

More than one phase function are used, as variations in either axes are given by $\alpha_{\theta}(\mathbf{x})$ and $\beta_{\theta}(\mathbf{x})$ respectively, whilst $\gamma_{\theta}(\mathbf{x})$ is considered by von Bülow and Sommer as a measure of 'degree of separability'. If the signal analysed takes the form $g(\mathbf{x})=g_{1}\left(x_{1}\right) g_{2}\left(x_{2}\right)$, i.e. the signal is separable, then they note that [17][p. 2849]: $\alpha_{0}(\mathbf{x})=\tan ^{-1}\left(\frac{\mathcal{H}_{1}\{g\}(\mathbf{x})}{g(\mathbf{x})}\right), \beta_{0}(\mathbf{x})=\tan ^{-1}\left(\frac{\mathcal{H}_{2}\{g\}(\mathbf{x})}{g(\mathbf{x})}\right)$, and $\gamma_{0}(\mathbf{x})=0$. Even if $g(\mathbf{x})$ is not separable the polar representation of equation (28) may be used to represent an arbitrary real-valued image $g(\mathbf{x})$.

Definition 2.8: The $\theta$-polar representations of $\mathrm{g}(\mathbf{x})$.

The $\theta$-polar representation of a real signal $g(\mathbf{x})$ is given by:

$$
\begin{aligned}
g(\mathbf{x})= & \Re\left\{\left|\widetilde{g}_{-\theta, \theta}^{(++)}(\mathbf{x})\right| e^{2 \pi i \widetilde{\alpha}_{-\theta, \theta}(\mathbf{x})} e^{2 \pi \boldsymbol{k} \widetilde{\gamma}_{-\theta, \theta}(\mathbf{x})} e^{2 \pi \boldsymbol{j} \widetilde{\beta}_{-\theta, \theta}(\mathbf{x})}\right\} \\
= & \left|\widetilde{g}_{-\theta, \theta}^{(++)}(\mathbf{x})\right|\left(\cos \left(2 \pi \widetilde{\alpha}_{-\theta, \theta}(\mathbf{x})\right) \cos \left(2 \pi \widetilde{\gamma}_{-\theta, \theta}(\mathbf{x})\right) \cos \left(2 \pi \widetilde{\beta}_{-\theta, \theta}(\mathbf{x})\right)\right. \\
& \left.+\sin \left(2 \pi \widetilde{\alpha}_{-\theta, \theta}(\mathbf{x})\right) \sin \left(2 \pi \widetilde{\gamma}_{-\theta, \theta}(\mathbf{x})\right) \sin \left(2 \pi \widetilde{\beta}_{-\theta, \theta}(\mathbf{x})\right)\right) .
\end{aligned}
$$

A separable structure in orientation $\theta$ will have $\gamma_{\theta}(\mathbf{x})=0$ and the signal is represented by:

$$
g(\mathbf{x})=\left|\widetilde{g}_{-\theta, \theta}^{(++)}(\mathbf{x})\right| \cos \left(2 \pi \widetilde{\alpha}_{-\theta, \theta}(\mathbf{x})\right) \cos \left(2 \pi \widetilde{\beta}_{-\theta, \theta}(\mathbf{x})\right) .
$$

Note that $\widetilde{\alpha}_{-\theta, \theta}(\mathbf{x})$ etc are determined by equation (29) but where now the $\theta$-hypercomplex extension of $\widetilde{g}_{-\theta}(\mathbf{x})$ is used as the basis of the representation. To see the utility of such a representation consider analysis of a signal separable in the rotated frame of reference, i.e.

$$
g(\mathbf{x})=\widetilde{g}_{-\theta}\left(\boldsymbol{r}_{-\theta} \mathbf{x}\right)=\widetilde{g}_{1,-\theta}\left(\left[\boldsymbol{r}_{-\theta} \mathbf{x}\right]_{1}\right) \widetilde{g}_{2,-\theta}\left(\left[\boldsymbol{r}_{-\theta} \mathbf{x}\right]_{2}\right) .
$$

Under the assumption that $g(\mathbf{x})$ satisfies equation (32), we determine that the $\theta$-hypercomplex extension of the signal is given (in terms of $\mathbf{x}^{\prime}=\boldsymbol{r}_{-\theta} \mathbf{x}$ ) by:

$$
\widetilde{g}_{-\theta, \theta}^{(++)}(\mathbf{x})=\widetilde{g}_{-\theta}\left(\mathbf{x}^{\prime}\right)+\boldsymbol{i} \mathcal{H}_{1}\left\{\widetilde{g}_{-\theta}\right\}\left(\mathbf{x}^{\prime}\right)+\boldsymbol{j} \mathcal{H}_{2}\left\{\widetilde{g}_{-\theta}\right\}\left(\mathbf{x}^{\prime}\right)+\boldsymbol{k} \mathcal{H}_{2} \mathcal{H}_{1}\left\{\widetilde{g}_{-\theta}\right\}\left(\mathbf{x}^{\prime}\right) .
$$

$\mathcal{H}_{1}\left\{\widetilde{g}_{-\theta}\right\}(\mathbf{x})=\mathcal{H}\left\{\widetilde{g}_{1,-\theta}\right\}\left(x_{1}\right) \widetilde{g}_{2,-\theta}\left(x_{2}\right)$,

$\mathcal{H}_{2}\left\{\widetilde{g}_{-\theta}\right\}(\mathbf{x})=\mathcal{H}\left\{\widetilde{g}_{2,-\theta}\right\}\left(x_{2}\right) \widetilde{g}_{1,-\theta}\left(x_{1}\right)$ and $\mathcal{H}_{2} \mathcal{H}_{1}\left\{\widetilde{g}_{-\theta}\right\}(\mathbf{x})=\mathcal{H}\left\{\widetilde{g}_{2,-\theta}\right\}\left(x_{2}\right) \mathcal{H}\left\{\widetilde{g}_{1,-\theta}\right\}\left(x_{1}\right)$. Hence, in our extended definition, if $g(\mathbf{x}) \in \mathbb{R}$ is separable in some rotated frame of reference $\mathbf{x}^{\prime}=\boldsymbol{r}_{-\theta} \mathbf{x}$, then $\widetilde{\gamma}_{-\theta, \theta}(\mathbf{x})=0$ for some $\theta \in[0, \pi / 2)$.

If a single component is present in $g(\mathbf{x})$ at $\mathbf{x}$ and the aim of the analysis was to determine the orientation of the separable structure in the image at this location, denoted $\theta^{\prime}$, then we calculate $\widetilde{g}_{-\theta, \theta}^{(++)}(\mathbf{x})$ at all angles $\theta \in[0, \pi / 2)$, and determine the value of $\theta^{\prime}$ by $\widetilde{\gamma}_{-\theta^{\prime}, \theta^{\prime}}(\mathbf{x})=0$. Once this has been done the signal is well described by its local magnitude, i.e.

$$
\left|\widetilde{g}_{-\theta, \theta}^{(++)}(\mathbf{x})\right|=\sqrt{\left[\left(\widetilde{g}_{1,-\theta}^{2}\left(x_{1}^{\prime}\right)+\mathcal{H}^{2}\left\{\widetilde{g}_{1,-\theta}\right\}\left(x_{1}^{\prime}\right)\right)\left(\widetilde{g}_{2,-\theta}^{2}\left(x_{2}^{\prime}\right)+\mathcal{H}^{2}\left\{\widetilde{g}_{2,-\theta}\right\}\left(x_{2}^{\prime}\right)\right)\right]}
$$

whilst local variation is best described by the two separable phase functions

$$
\widetilde{\alpha}_{-\theta, \theta}(\mathbf{x})=\tan ^{-1}\left(\mathcal{H}\left\{\widetilde{g}_{1,-\theta}\right\}\left(x_{1}^{\prime}\right) / \widetilde{g}_{1,-\theta}\left(x_{1}^{\prime}\right)\right), \quad \widetilde{\beta}_{-\theta, \theta}(\mathbf{x})=\tan ^{-1}\left(\mathcal{H}\left\{\widetilde{g}_{2,-\theta}\right\}\left(x_{2}^{\prime}\right) / \widetilde{g}_{2,-\theta}\left(x_{2}^{\prime}\right)\right) .
$$


The local frequency is found in each of the two axes as $\frac{\partial}{\partial x_{1}^{\prime}} \widetilde{\alpha}_{-\theta, \theta}(\mathbf{x})$ and $\frac{\partial}{\partial x_{2}^{\prime}} \widetilde{\beta}_{-\theta, \theta}(\mathbf{x})$.

Proposition 1: Orthogonality of the signal and the HTs.

The original signal $g(\mathbf{x})$ and $g_{H}^{(s)}(\mathbf{x}), s=1,2,3$, are all mutually orthogonal.

Proof: This is a trivial consequence of the orthogonality of a 1-D HT. Furthermore we note that as $\widetilde{g}_{-\theta}(\mathbf{x})$ is orthogonal to $\widetilde{g}_{-\theta ; H}^{(s)}(\mathbf{x}), s=1,2,3$, we find that $g(\mathbf{x})$ is orthogonal to $\widetilde{g}_{-\theta ; H}^{(s)}\left(\boldsymbol{r}_{-\theta} \mathbf{x}\right), s=1,2,3$.

Proposition 2: Norm of the hypercomplex components.

The $g_{H}^{(s)}, s=1,2,3$, have the same norm as $g(\mathbf{x})$.

Proof: This is a direct result following from the norm of the 1-D HT of a real-valued function [23][p. 4].

Hence in summary: $\mathscr{P} u\left\{g^{(++)}(\mathbf{x})\right\}$ defines a quaternionic object where each real-valued function multiplying the quaternions $\boldsymbol{i}, \boldsymbol{j}$ and $\boldsymbol{k}$ can be considered as objects with the same spatial/spatial frequency support as $g(\mathbf{x})$, as the HT constructs an object with the same time-frequency support as the original signal [64]. Note that all of $\left\{g_{-\theta, \theta}^{(s)}(\mathbf{x})\right\}$ for $s=1,2, T$ are mutually orthogonal. $\mathscr{P} u\left\{g_{-\theta, \theta}^{(++)}(\mathbf{x})\right\}$ constructs a quaternionic object with the same spatial and spatial frequency support as $g(\mathbf{x})$, where the relative magnitudes of the components specifies the structure of $g(\mathbf{x})$ in a rotated frame.

\section{G. The monogenic signal}

The hypercomplex signal, once extended to the $\theta$-hypercomplex signals recognizes locally separable structure, and can if the signal is calculated for all $\theta \in[0,2 \pi)$, determine locally unidirectional structure at a given orientation. However, this does require the calculation of the hypercomplex extension at all $\theta$. A hyperanalytic extension for locally unidirectional structure, or plane waves, at any given spatial point can also be made. Felsberg \& Sommer, [25], introduced a hyperanalytic extension in image processing suitable for this purpose. See also an early work by Dixon [39], where monogenic functions were denoted 'pure Hamiltonian' functions and the discussion by Duffin in [65]. This approach starts from the $d$-D Poisson kernels [38], a $d$-D version of equation (9), defined for any dimension $d \geq 1$ :

$$
p(\mathbf{x}, y)=c_{d} \frac{y}{\left[|\mathbf{x}|^{2}+y^{2}\right]^{\frac{d+1}{2}}}, \quad q_{l}(\mathbf{x}, y)=c_{d} \frac{x_{l}}{\left[|\mathbf{x}|^{2}+y^{2}\right]^{\frac{d+1}{2}}} \quad c_{d}=\frac{\Gamma\left(\frac{n+1}{2}\right)}{\pi^{\frac{n+1}{2}}}, l=1, \ldots, d .
$$

An extra set of $d+1$ functions

$$
u_{R ; g}(\mathbf{x}, y)=(p(\cdot, y) * * g(\cdot))(\mathbf{x}), \quad v_{R ; g}^{(l)}(\mathbf{x}, y)=\left(q_{l} * * g\right)(\mathbf{x}, y),
$$

are defined from $g(\mathbf{x})$. For $d=2,\left\{u_{R ; g}(\mathbf{x}, y), v_{R ; g}^{(1)}(\mathbf{x}, y), v_{R ; g}^{(2)}(\mathbf{x}, y)\right\}$ satisfy an alternative set of generalized Cauchy-Riemann equations, with only a single auxiliary variable $y$. These generalized Cauchy-Riemann equations are called the Riesz system, see [38][p. 234].

Definition 2.9: The monogenic function.

Any solution of the Riesz system in the upper half-space $(y>0)$ is denoted a monogenic function [66][p. 35]. Note that if $k^{(+)}(\mathbf{x}, y)$ is a monogenic function then $k^{(+) *}(\mathbf{x}, y)$ is a solution of the Riesz system in the lower half-space $(y<0)$, and will be denoted an anti-monogenic function, with notation $k^{(-)}(\mathbf{x}, y)$.

For $d=2$, if $k_{R ; g}^{(+)}(\mathbf{x}, y)=u_{R ; g}(\mathbf{x}, y)+\boldsymbol{i} v_{R ; g}^{(1)}(\mathbf{x}, y)+\boldsymbol{j} v_{R ; g}^{(2)}(\mathbf{x}, y)$ then it is a monogenic function [38][p. 235]. In the limit of $y \rightarrow 0^{+}[38]:$

$$
\lim _{y \rightarrow 0^{+}} u_{R ; g}(\mathbf{x}, y)=g(\mathbf{x}), \quad \lim _{y \rightarrow 0^{+}} v_{R ; g}^{(l)}(\mathbf{x}, y)=g(\mathbf{x}) * \ldots * r_{l}(\mathbf{x})=\mathcal{R}_{l} g(\mathbf{x})=g_{R}^{(l)}(\mathbf{x}),
$$

where the quantities $r_{l}(\mathbf{x})$ and $R_{l}(\mathbf{f})$ are given by:

$$
r_{l}=c_{n} \frac{x_{l}}{|\mathbf{x}|^{n+1}}, \quad R_{l}(\mathbf{f})=-\boldsymbol{j} \frac{f_{l}}{f}, \quad R_{1, Q}(\boldsymbol{q})=-\boldsymbol{i} \frac{q_{1}}{q}, \quad R_{2, Q}(\mathbf{f})=-\boldsymbol{j} \frac{q_{2}}{q} \quad l=1,2 .
$$

$\left\{r_{l}(\mathbf{x})\right\}$ are the Riesz kernels for $d \geq 2$, and the FT and QFTs of the Riesz kernels are given by [27], [38].

Definition 2.10: The Riesz transform.

The Riesz transform of a signal $g(\mathbf{x}) \in L^{2}\left(\mathbb{R}^{2}\right)$ is defined by operator $\mathcal{R}$

$$
\mathcal{R}\{g\}(\mathbf{x})=\boldsymbol{i} \mathcal{R}\{g\}(\mathbf{x})+\boldsymbol{j} \mathcal{R}_{2}\{g\}(\mathbf{x}), \quad \mathcal{R}_{l}\{g\}(\mathbf{x})=g_{R}^{(l)}(\mathbf{x})=\left(r_{l} * * g\right)(\mathbf{x}), l=1,2 .
$$


Felsberg \& Sommer [25] recently introduced the monogenic signal into image processing by combining $g(\mathbf{x})$ with $\mathcal{R}\{g\}(\mathbf{x})$ :

Definition 2.11: The monogenic signal.

The monogenic, and anti-monogenic signal of real signal $g(\mathbf{x})$, are defined by applying operator $\mathcal{M}^{ \pm}$to signal $g(\mathbf{x})$ :

$$
g^{( \pm)}(\mathbf{x})=\mathcal{M}^{ \pm}\{g\}(\mathbf{x})=g(\mathbf{x}) \pm \mathcal{R}\{g\}(\mathbf{x})=g(\mathbf{x}) \pm\left[\boldsymbol{i} g_{R}^{(1)}(\mathbf{x})+\boldsymbol{j} g_{R}^{(2)}(\mathbf{x})\right] .
$$

Note that $\mathcal{R}$, and $g_{R}^{(l)}(\mathbf{x})$ are defined by equation $(38)$.

Letting $y \rightarrow 0^{+}$the monogenic signal is retrieved from the monogenic function, and so we find that $g^{(+)}(\mathbf{x})$ is a hyperanalytic signal, $c f$ definition (2.2). See also the careful discussion in [66][p. 32-37] on monogenic signals and the generalized Cauchy-Riemann equations.

Only one auxiliary variable is introduced when constructing the Riesz components, linked with the norm of $\mathbf{x}$, and the monogenic and anti-monogenic signals play the roles in 2-D of the analytic and anti-analytic signals in 1-D. To accommodate that the observed image is not observed in the direction of the local variation, we introduce here the concept of a $\theta$-monogenic and anti-monogenic signal.

Definition 2.12: The $\theta$-monogenic signal.

The $\theta$-monogenic, and $\theta$-anti-monogenic signal of real signal $g(\mathbf{x})$ are for any value of a rotation $\theta \in[0,2 \pi)$ given by:

$$
\begin{aligned}
g_{\theta}^{( \pm)}(\mathbf{x}) & =R_{\theta} \mathcal{M}^{ \pm}\{g\}(\mathbf{x})=\mathcal{M}_{\theta}^{ \pm}\{g\}(\mathbf{x})=g\left(\boldsymbol{r}_{-\theta} \mathbf{x}\right) \pm\left[\boldsymbol{i} R_{\theta} \mathcal{R}_{1} g(\mathbf{x})+\boldsymbol{j} R_{\theta} \mathcal{R}_{2} g(\mathbf{x})\right] \\
& =g\left(\boldsymbol{r}_{-\theta} \mathbf{x}\right) \pm\left[\boldsymbol{i} g_{R}^{(1)}\left(\boldsymbol{r}_{-\theta} \mathbf{x}\right)+\boldsymbol{j} g_{R}^{(2)}\left(\boldsymbol{r}_{-\theta} \mathbf{x}\right)\right]=g_{\theta}(\mathbf{x}) \pm\left[\boldsymbol{i} g_{\theta ; R}^{(1)}(\mathbf{x})+\boldsymbol{j} g_{\theta ; R}^{(2)}(\mathbf{x})\right]
\end{aligned}
$$

thus defining the components $g_{\theta ; R}^{(s)}(\mathbf{x})$ for $s=1,2$.

Note that

$$
\mathcal{F}\left\{g_{R}^{(1)}\left(\boldsymbol{r}_{-\theta} \mathbf{x}\right)\right\}=-\boldsymbol{j} \cos (\phi-\theta) G\left(\boldsymbol{r}_{-\theta} \mathbf{f}\right), \mathcal{F}\left\{g_{R}^{(2)}\left(\boldsymbol{r}_{-\theta} \mathbf{x}\right)\right\}=-\boldsymbol{j} \sin (\phi-\theta) G\left(\boldsymbol{r}_{-\theta} \mathbf{f}\right) .
$$

Theorem 1: $\theta$-monogenic functions and their monogenicity.

The $\theta$-monogenic and the $\theta$-anti-monogenic signals $g_{\theta}^{( \pm)}(\mathbf{x})$ are both the limits of sets of functions satisfying the Riesz system of equations in a rotated frame of reference.

Proof: See section [-B Thus a $\theta$-monogenic signal can be considered to be the limit of a monogenic function in the rotated frame of reference. For consistency with our notation so far, if $\theta=0$, the signal is denoted $g^{( \pm)}(\cdot)$ without subscript. Furthermore we may note that

$$
\begin{aligned}
G_{\theta}^{( \pm)} & =G\left(\boldsymbol{r}_{-\theta} \mathbf{f}\right) \pm\left(-\boldsymbol{k} \cos (\phi-\theta) G\left(\boldsymbol{r}_{-\theta} \mathbf{f}\right)+\sin (\phi-\theta) G\left(\boldsymbol{r}_{-\theta} \mathbf{f}\right)\right) \\
& =[1 \pm \sin (\phi-\theta) \mp \boldsymbol{k} \cos (\phi-\theta)] G\left(\boldsymbol{r}_{-\theta} \mathbf{f}\right) .
\end{aligned}
$$

If the Fourier transform of a function can be written in the form of equation (42) it can be directly deduced that the function corresponds to the $\theta$-monogenic image of $g(\mathbf{x})$. Thus just like we intend to use equation (24) to recognize a quaternionic object as corresponding to a $\theta$-hypercomplex object, equation (42) can be used to determine if a quaternionic object is $\theta$-monogenic, and what real-valued object plays the role of $g(\mathbf{x})$.

A real-valued image can be decomposed into a $\theta$-monogenic and $\theta$-anti-monogenic component. Using $\widetilde{g}_{\theta}(\mathbf{x})$ defined in equation (26) we represent $g(\mathbf{x})$ by:

$$
\begin{aligned}
\widetilde{g}_{-\theta, \theta}^{( \pm)}(\mathbf{x}) & =\widetilde{g}_{-\theta}\left(\boldsymbol{r}_{-\theta} \mathbf{x}\right) \pm\left(\boldsymbol{i} \widetilde{g}_{-\theta ; R}^{(1)}\left(\boldsymbol{r}_{-\theta} \mathbf{x}\right)+\boldsymbol{j} \widetilde{g}_{-\theta ; R}^{(2)}\left(\boldsymbol{r}_{-\theta} \mathbf{x}\right)\right) \\
& =g(\mathbf{x}) \pm\left(\boldsymbol{i} \widetilde{g}_{-\theta ; R}^{(1)}\left(\boldsymbol{r}_{-\theta} \mathbf{x}\right)+\boldsymbol{j} \widetilde{g}_{-\theta ; R}^{(2)}\left(\boldsymbol{r}_{-\theta} \mathbf{x}\right)\right) .
\end{aligned}
$$

$\mathcal{R}_{1} R_{-\theta} g(\mathbf{x})=\cos (\theta) R_{-\theta} \mathcal{R}_{1} g(\mathbf{x})+\sin (\theta) R_{-\theta} \mathcal{R}_{2} g(\mathbf{x})$, and as noted by [38][p. 241]:

$$
\begin{aligned}
& \widetilde{g}_{-\theta ; R}^{(1)}\left(\boldsymbol{r}_{-\theta} \mathbf{x}\right)=R_{\theta} \mathcal{R}_{1} R_{-\theta} g(\mathbf{x})=\cos (\theta) \mathcal{R}_{1} g(\mathbf{x})+\sin (\theta) \mathcal{R}_{2} g(\mathbf{x}) \neq \mathcal{R}_{1} g(\mathbf{x}) \\
& \widetilde{g}_{-\theta ; R}^{(2)}\left(\boldsymbol{r}_{-\theta} \mathbf{x}\right)=R_{\theta} \mathcal{R}_{2} R_{-\theta} g(\mathbf{x})=-\sin (\theta) \mathcal{R}_{1} g(\mathbf{x})+\cos (\theta) \mathcal{R}_{2} g(\mathbf{x}) \neq \mathcal{R}_{2} g(\mathbf{x}) .
\end{aligned}
$$


The rotation operator, $R_{\theta}$, and the Riesz transform operators, $\mathcal{R}_{l}, l=1,2$, do not commute, and so $\widetilde{g}_{\theta, \theta}^{( \pm)}(\mathbf{x}) \neq$ $g^{( \pm)}(\mathbf{x})$.

Definition 2.13: $\theta$-monogenic: monogenic/anti-monogenic decomposition.

The decomposition of real-valued $g(\mathbf{x})$ into two quaternionic components $\widetilde{g}_{-\theta, \theta}^{(+)}(\mathbf{x})$ and $\widetilde{g}_{-\theta, \theta}^{(-)}(\mathbf{x})$ :

$$
g(\mathbf{x})=\frac{1}{2}\left[\widetilde{g}_{-\theta, \theta}^{(+)}(\mathbf{x})+\widetilde{g}_{-\theta, \theta}^{(-)}(\mathbf{x})\right],
$$

will be denoted the $\theta$-monogenic/anti-monogenic decomposition of $g(\mathbf{x})$, where the two components are given by: $\widetilde{g}_{-\theta, \theta}^{( \pm)}(\mathbf{x})=g(\mathbf{x}) \pm\left(\boldsymbol{i} \widetilde{g}_{-\theta ; R}^{(1)}\left(\boldsymbol{r}_{-\theta} \mathbf{x}\right)+\boldsymbol{j} \widetilde{g}_{-\theta ; R}^{(2)}\left(\boldsymbol{r}_{-\theta} \mathbf{x}\right)\right)$. This is a natural decomposition of a locally 1-D signal more naturally observed in a rotated frame of reference. Equation (45) forms a 2-D extension of the decomposition of a real-valued object into a quaternionic form in analogue with the analytic/anti-analytic decomposition of a 1-D signal, see [41].

\section{H. Properties of the monogenic signal}

Again, it is natural to represent a hyperanalytic function in terms of amplitude and local structure, and so [47], [66], [67] represent the monogenic signal in terms of an amplitude and a phase. A single phase only is used to describe variation as the monogenic representation corresponds to a local plane wave structure. The phase represents the local period in the direction of variation determined from the signal.

Definition 2.14: The polar representation of the $\theta$-monogenic signal.

The $\theta$-monogenic and anti-monogenic images will be represented in terms of the amplitude, phase and direction by

$$
g_{\theta}^{( \pm)}(\mathbf{x})=\left|g_{\theta}^{(+)}(\mathbf{x})\right| e^{ \pm 2 \pi e_{\nu_{\theta}}(\mathbf{x}) \phi_{\theta}(\mathbf{x})},
$$

where the amplitude, phase and direction are given by

$$
\begin{aligned}
\left|g_{\theta}^{(+)}(\mathbf{x})\right| & =\sqrt{\left[g_{\theta}(\mathbf{x})\right]^{2}+\left[g_{\theta ; R}^{(1)}(\mathbf{x})\right]^{2}+\left[g_{\theta ; R}^{(2)}(\mathbf{x})\right]^{2}}, \quad e_{\nu_{\theta}}(\mathbf{x})=\left[\boldsymbol{i} \cos \nu_{\theta}(\mathbf{x})+\boldsymbol{j} \sin \nu_{\theta}(\mathbf{x})\right], \\
\nu_{\theta}(\mathbf{x}) & =\tan ^{-1}\left(\frac{g_{\theta ; R}^{(2)}(\mathbf{x})}{g_{\theta ; R}^{(1)}(\mathbf{x})}\right), \quad \phi_{\theta}(\mathbf{x})=\frac{1}{2 \pi} \tan ^{-1}\left(\operatorname{sgn}\left(g_{\theta ; R}^{(2)}(\mathbf{x})\right) \frac{\sqrt{\left[g_{\theta ; R}^{(1)}(\mathbf{x})\right]^{2}+\left[g_{\theta ; R}^{(2)}(\mathbf{x})\right]^{2}}}{g_{\theta}(\mathbf{x})}\right) .
\end{aligned}
$$

The $\operatorname{sgn}(\cdot)$ added to the definition of $\phi_{\theta}(\mathbf{x})$ to appropriately determine the range of $\phi_{\theta}(\mathbf{x})$. Note that the $\theta$-monogenic and $\theta$-anti-monogenic images of a real image $g(\mathbf{x})$ have the same modulus and phase. $g_{\theta}^{(+) *}(\mathbf{x})=g_{\theta}^{(-)}(\mathbf{x})$.

Definition 2.15: The $\theta$-polar representation of $\mathrm{g}(\mathbf{x})$.

The $\theta$-polar representation of a real signal $g(\mathbf{x})$ is given by:

$$
g(\mathbf{x})=\Re\left\{\widetilde{g}_{-\theta, \theta}^{( \pm)}(\mathbf{x})\right\}=\left|\widetilde{g}_{-\theta, \theta}^{( \pm)}(\mathbf{x})\right| \cos \left(2 \pi \widetilde{\phi}_{-\theta, \theta}(\mathbf{x})\right) .
$$

Thus locally a signal is described by its magnitude $\left|\widetilde{g}_{-\theta, \theta}^{(+)}(\mathbf{x})\right|$ and the local structure of the signal is determined by $\widetilde{\phi}_{-\theta, \theta}(\mathbf{x})$, from which an instantaneous frequency is determined by $\left|\nabla \widetilde{\phi}_{-\theta, \theta}(\mathbf{x})\right|$. From equation (44) it clearly follows that: $\left|\widetilde{g}_{-\theta, \theta}^{( \pm)}(\mathbf{x})\right|=\left|g^{( \pm)}(\mathbf{x})\right|, \widetilde{\phi}_{-\theta, \theta}(\mathbf{x})=\widetilde{\phi}(\mathbf{x})$, but $e_{\widetilde{\nu}_{-\theta, \theta}}(\mathbf{x}) \neq e_{\nu}(\mathbf{x})$. The monogenic representation of $g(\mathbf{x})$ is thus equivalent to the $\theta$-monogenic representation, unlike the case of the hypercomplex and $\theta$-hypercomplex representation, that are clearly distinct. The intuitive understanding of these relationships is that the monogenic signal is constructing a plane wave representation at spatial point $\mathbf{x}$, where the direction of the plane wave is determined from the signal. No matter what axes we construct, the local plane wave representation in the amplitude and period (phase) remain the same, but the parameterization of the direction changes with the axes. The reason for introducing the $\theta$-monogenic signal is that the calculations of the properties of the CWT are simplified. Hence for convenience the notation $\left|\widetilde{g}_{-\theta, \theta}^{( \pm)}(\mathbf{x})\right|$ and $\widetilde{\phi}_{-\theta, \theta}(\mathbf{x})$, are retained, even if both are in some sense redundant.

Proposition 3: Orthogonality of the signal and the Riesz transform.

A real image $g(\mathbf{x})$ is orthogonal to each component of its Riesz transform $\mathcal{R}$. However, the two components of the Riesz transform, $g_{R}^{(1)}(\mathbf{x}), g_{R}^{(2)}(\mathbf{x})$ are in general not orthogonal. 
Proof: This is a direct generalisation of the $n=1$ case where the HT is orthogonal to the original signal. Indeed, exploiting the Hermitian symmetry of the FT of a real image $G^{*}(\mathbf{f})=G(-\mathbf{f})$, we have

$$
\left\langle g_{R}^{(1)}, g\right\rangle=\int d^{2} \mathbf{f} j \frac{f_{1}}{f} G^{*}(\mathbf{f}) G(\mathbf{f})=\int d^{2} \mathbf{f} j \frac{f_{1}}{f} G(-\mathbf{f}) G(\mathbf{f})=0 .
$$

The function $G(-\mathbf{f}) G(\mathbf{f}) / f$ is even-even (ee) with respect to $f_{1}$ and $f_{2}$ whereas $f_{1}$ itself is obviously oddeven (oe). Since the integration is over the entire $E_{2}$ plane, it is zero. By the same argument $\left\langle g_{R}^{(2)}, g\right\rangle=0$ and therefore $\langle\mathcal{R} g, g\rangle=0$. Furthermore we note that $\widetilde{g}_{-\theta}(\mathbf{x})$ is orthogonal to $\widetilde{g}_{-\theta}^{(s)}(\mathbf{x})$, and thus $g(\mathbf{x})$ is orthogonal to $\widetilde{g}_{-\theta}^{(s)}\left(\boldsymbol{r}_{-\theta} \mathbf{x}\right), s=1,2$. However, the two components of the Riesz transform itself are not in general orthogonal:

$$
\left\langle g_{R}^{(2)}, g_{R}^{(1)}\right\rangle=\int \frac{f_{1} f_{2}}{f^{2}} G(\mathbf{f}) G(-\mathbf{f}) d^{2} \mathbf{f} \neq 0 .
$$

The natural way to view the monogenic image is as consisting of two orthogonal components, $g(\mathbf{x})$ and $\mathcal{R} g(\mathbf{x})$.

Proposition 4: Orthogonality of the components of the Riesz transform of an isotropic function.

If the signal $g(\mathbf{x})$ is radially symmetric and real, then the integrand in (49) is

$$
\left\langle g_{R}^{(2)}, g_{R}^{(1)}\right\rangle=0 .
$$

Proof: $G(\mathbf{f})=G(f)$, and radially symmetric as well as real, whilst $f_{1} f_{2}$ is odd with respect to either $f_{1}$ or $f_{2}$. Thus $\left\langle g_{R}^{(2)}, g_{R}^{(1)}\right\rangle=0$. Note that for an isotropic signal $g(\mathbf{x})=\widetilde{g}_{-\theta}(\mathbf{x})$ and so $g(\mathbf{x})$ is orthogonal to $\widetilde{g}_{-\theta, H}^{(s)}(\mathbf{x})$.

Proposition 5: Norm of the Riesz components.

The norm of the Riesz components is given by (as noted by Felsberg \& Sommer [25][p. 3140]):

$$
\left\langle g_{R}^{(s)}, g_{R}^{(s)}\right\rangle=\int \frac{f_{s}^{2}}{f^{2}} G(-\mathbf{f}) G(\mathbf{f}) d^{2} \mathbf{f}, s=1,2, \quad\left\langle g_{R}^{(1)}, g_{R}^{(1)}\right\rangle+\left\langle g_{R}^{(2)}, g_{R}^{(2)}\right\rangle=\langle g, g\rangle .
$$

If furthermore $g(\mathbf{x})$ is radially symmetric then

$$
\left\langle g_{R}^{(s)}, g_{R}^{(s)}\right\rangle=\frac{1}{2}\langle g, g\rangle, s=1,2 .
$$

These relationships were proved and used in [44] in the special case of the isotropic multiple Morse wavelets. In summary $\mathcal{R} g(\mathbf{x})$ is orthogonal to $g(\mathbf{x})$, has the same frequency support, as well as the same total norm as $g(\mathbf{x})$ and exhibits similar spatial decay as $g(\mathbf{x})$ (or at least as similar as $\mathcal{H} g\left(x_{1}\right)$ does to $g\left(x_{1}\right)$.) Hence as $\mathcal{H} g\left(x_{1}\right)$ is considered as having the same time-frequency structure presence as $g\left(x_{1}\right)$ (see for example Boashash [64]) and we consider $\mathcal{R} g(\mathbf{x})$ as having the same spatial and spatial frequency support as $g(\mathbf{x})$.

\section{The monogenic signal and the UQFT}

The monogenic signal determines the directional preference of a given real signal at a given point in space, $\mathbf{x}$. Let us discuss this aspect in some more detail. As the monogenic representation is suitable for a plane wave field $g(\mathbf{x})$, consider the representation given to a purely unidirectional signal $g_{U}(\mathbf{x})$. By assuming that $g_{U}(\mathbf{x})$ purely experiences variation in a given direction, implies that it can be represented as a superposition of plane waves with direction $\nu$. Then for some given constant $\boldsymbol{n}=\left[\begin{array}{ll}\cos (\nu) & \sin (\nu)\end{array}\right]$ :

$$
g_{U}(\mathbf{x})=\int_{0}^{\infty} G_{U}(f) \cos \left(2 \pi f \boldsymbol{n}^{T} \mathbf{x}\right) d f .
$$

Define the unit quaternion $\boldsymbol{e}_{\nu}=\cos (\nu) \boldsymbol{i}+\sin (\nu) \boldsymbol{j}$. We calculate the monogenic signal of $g_{U}(\mathbf{x})$, and determine that:

$$
\begin{aligned}
g_{U}^{(+)}(\mathbf{x}) & =\int_{0}^{\infty} G_{U}(f) \exp \left(2 \pi \boldsymbol{e}_{\nu} f \boldsymbol{n}^{T} \mathbf{x}\right) d f, \quad \nu=\frac{g_{U ; R}^{(2)}(\mathbf{x})}{g_{U ; R}^{(1)}(\mathbf{x})} \\
& =\int_{0}^{\infty} G_{U}(f) \cos \left(2 \pi f \boldsymbol{n}^{T} \mathbf{x}\right) d f+[\boldsymbol{i} \cos (\nu)+\boldsymbol{j} \sin (\nu)] \int_{0}^{\infty} G_{U}(f) \sin \left(2 \pi f \boldsymbol{n}^{T} \mathbf{x}\right) d f .
\end{aligned}
$$

This result follows from the monogenic extension of a sinusoid given in [25], and the global orientation of the globally directional signal may be determined from the monogenic signal by calculating the ratio of the two Riesz 
transforms. Let us now demonstrate how $g_{U}^{(+)}(\mathbf{x})$ is related to the UQFT in $\boldsymbol{e}_{\nu}$. It may be shown, again using the monogenic extension of a sinusoid given in [25], that $G_{U, \boldsymbol{e}_{\nu}}^{(+)}(\mathbf{f})=G_{U}(f), f>0$, so that the spectral support of the UQFT of the signal in $e_{\nu}$ is one-sided, and limited to the positive frequencies. Thus an equivalent to equation (7) can be derived that corresponds to an alternative to $G_{Q}^{(++)}(\mathbf{q})=0$. The result is to be expected, as the plane waves are really a 1-D feature embedded in a 2-D space. If the signal corresponds to unidirectional variation only, the monogenic signal is extracting the orientation, and lives in a half-plane, whilst the anti-monogenic signal lives in the second half-plane. Calculating the partial HT in the correct direction will yield an equivalent representation to using the monogenic signal, but the advantage of using the latter is that the direction is retrieved directly from the observed image without having to perform HTs at all values of $\theta$. The assumption of global unidirectionality is very strict, and constructing a local description of the signal will be essential for the performance of the analysis method.

\section{WAVELET ANALYSIS}

Wavelets provide a method of constructing a local decomposition of an observed image, that for many classes of images constitute of decomposition coefficients mainly of negligible magnitude. Furthermore, due to the definition of the wavelet coefficients, it is also frequently easier to interpret the non-negligible coefficients, rather than trying to disentangle the full behaviour of an image in the original spatial domain. The interpretation of the coefficients relies on the choice of mother wavelet. To be able to satisfy the reconstruction formula of equation (3) for any arbitrary function $g(\mathbf{x}) \in L^{2}\left(\mathbb{R}^{2}\right)$, the mother wavelet function, denoted $\psi(\mathbf{x})$, must satisfy the two conditions of

$$
0<c_{\psi}<\infty, \text { where } c_{\psi}=(2 \pi)^{2} \int_{E_{2}} \frac{|\Psi(\mathbf{f})|^{2}}{f^{2}} d^{2} \mathbf{f}, \quad \int_{E_{2}}|\psi(\mathbf{x})|^{2} d^{2} \mathbf{x}=1 .
$$

Thus the mother wavelet is constrained to be both a spatially local function, and to be mainly supported over some range of frequencies not including the origin, i.e. the function is oscillatory and zero-mean. Without loss of generality, center the mother wavelet function in space to $\mathbf{x}=\mathbf{0}$, and assume that $\Psi(\mathbf{f})$ is maximum at $\mathbf{f}=$ $\mathbf{f}_{0}=f_{0}\left[\begin{array}{ll}\cos \left(\phi_{0}\right) & \sin \left(\phi_{0}\right)\end{array}\right]^{T}$. Of course the possible structure $\psi(\mathbf{x})$ may exhibit whilst still satisfying equation (55) is very variable, and even more so in 2-D compared to 1-D. For example, the wavelets developed in [20], [21], [43], [68] are complex and describe directional plane waves, as do the wavelets of [1], whilst a great deal of applications that rely on perfect reconstruction use discrete filter bank separable wavelets [4], and a third possible option corresponds to using radial functions, as described in Antoine et al. [13], Metikas \& Olhede [44] and also by Farge [69]. Visually, in a contour plot description of the wavelets, highly directional plane wavelets have the appearance of local maxima corresponding to evenly spaced lines with a given normal, separable wavelets have local maxima and minima in a checkerboard patter, and isotropic wavelets correspond to local maxima appearing in repeating ring-shapes with approximately an even spacing between the rings. The 'optimal' choice of wavelet naturally depends on the application in question.

This article aims to provide classes of mother wavelet functions so that the wavelet coefficients calculated are hyperanalytic signals in the index $\mathbf{b}$. Additionally energy in the observed image should be separated to the correct decomposition coefficient, and this corresponds to using wavelets that are essentially supported over a limited region of space and spatial frequency. To satisfy the latter restriction wavelets that are well-localised [13], [44], are often used. Local structure will be represented in terms of interrelationships between coefficients in a vectorvalued representation at each local decomposition coefficient index point. To construct a four-vector (quaternionic) description of local structure, we use wavelets of the form:

$$
\psi(\mathbf{x})=\psi^{(r)}(\mathbf{x})+\boldsymbol{i} \psi^{(i)}(\mathbf{x})+\boldsymbol{j} \psi^{(j)}(\mathbf{x})+\boldsymbol{k} \psi^{(k)}(\mathbf{x}),
$$

where each $\psi^{(s)} \in L^{2}\left(\mathbb{R}^{2}\right)$ for $s=r, i, j, k$. Thus for any $\mathbf{x} \in \mathbb{R}^{2}$ it follows that $\psi(\mathbf{x}) \in \mathbb{H}$.

In general we construct $\psi(\mathbf{x})$ from $\psi^{(r)}(\mathbf{x})$ and will denote the construction operator by $\psi(\mathbf{x})=\mathcal{W} \psi^{(r)}(\mathbf{x})$. If the observed image can locally be described as a plane wave, as is the case in equation (13), then the local monogenic description of the signal is appropriate, and the CWT will be constructed to correspond to a local UQFT ( $c f$ equation (18).) If on the other hand, local separable variations in an appropriate frame of reference are present like the signal modelled by equation (15), then a local version of the QFT, $c f$ equation (16), should be used for analysis. 
Naturally the whole family of wavelet coefficients constructed from the family of wavelet functions must enjoy suitable properties.

Definition 3.1: Members of the quaternionic wavelet family.

An arbitrary member of the quaternionic wavelet family is defined by

$$
\psi_{\boldsymbol{\xi}}(\mathbf{x})=U_{\boldsymbol{\xi}} \psi(\mathbf{x})=\psi_{\boldsymbol{\xi}}^{(r)}(\mathbf{x})+\boldsymbol{i} \psi_{\boldsymbol{\xi}}^{(i)}(\mathbf{x})+\boldsymbol{j} \psi_{\boldsymbol{\xi}}^{(j)}(\mathbf{x})+\boldsymbol{k} \psi_{\boldsymbol{\xi}}^{(k)}(\mathbf{x}) .
$$

The construction corresponds to individually translating, scaling and rotating the three components of the quaternionic function. To define any member of the quaternionic wavelet family, that is $\psi_{\boldsymbol{\xi}}(\mathbf{x})$, each of the real wavelet functions that combine to make up the quaternionic wavelet is scaled, rotated and shifted so that $\psi_{\boldsymbol{\xi}}(\mathbf{x})=$ $U_{\boldsymbol{\xi}} \mathcal{W} \psi^{(r)}(\mathbf{x})$. Note that in general $\psi_{\boldsymbol{\xi}}(\mathbf{x}) \neq \mathcal{W} U_{\boldsymbol{\xi}} \psi^{(r)}(\mathbf{x})$, and some care must be taken when noting the properties of the members of the wavelet family. Also the properties of the CWT coefficients must be considered with some care, as in general neither is $w_{\mathcal{W} \psi^{(r)}}(\boldsymbol{\xi} ; g)=\mathcal{W} w_{\psi^{(r)}}(\boldsymbol{\xi} ; g)$, where $\mathcal{W}$ is implemented in b. It is very important that the wavelet coefficients are interpretable, as they may be considered as a local projection of the image, and they, rather than any other given quantity, will be the basis for analysis of a given image. We shall discuss the properties enjoyed by the wavelets as well as the CWT coefficients in detail.

\section{A. Hypercomplexing wavelets}

The QFT decomposes an image into sinusoids, where the parity of variations in both $x_{1}$ and $x_{2}$ can be considered separately via the $i, j$ and $\boldsymbol{k}$ components of the QFT. From equation (16) we can note that the construction of the QFT is highly non-trivial, as $g(\mathbf{x})$ is left-multiplied by $\exp \left(-2 \pi \boldsymbol{i} x_{1} q_{1}\right)$ and right-multiplied by $\exp \left(-2 \pi \boldsymbol{j} x_{2} q_{2}\right)$, so if a quaternionic $g(\mathbf{x})$ is decomposed in the QFT coefficients, then the transform cannot be described in terms of an inner product of $g(\mathbf{x})$ with another function. If $g(\mathbf{x}) \in \mathbb{R}$ then $G_{Q}(\mathbf{q})=$ $\int_{-\infty}^{\infty} \int_{-\infty}^{\infty} g(\mathbf{x}) \exp \left(-2 \pi \boldsymbol{i} x_{1} q_{1}\right) \exp \left(-2 \pi \boldsymbol{j} x_{2} q_{2}\right)$

$d^{2} \mathbf{x}$, and the QFT may thus be expressed as an inner product between $\left[\exp \left(-2 \pi \boldsymbol{i} x_{1} q_{1}\right) \exp \left(-2 \pi \boldsymbol{j} x_{2} q_{2}\right)\right]^{*}$ and $g(\mathbf{x})$. Note that

$$
\left(e^{-2 \pi i q_{1} x_{1}} e^{-2 \pi \boldsymbol{j} q_{2} x_{2}}\right)^{*}=\vartheta^{(e e)}(\mathbf{x})+\boldsymbol{i} \vartheta^{(o e)}(\mathbf{x})+\boldsymbol{j} \vartheta^{(e o)}(\mathbf{x})-\boldsymbol{k} \vartheta^{(o o)}(\mathbf{x}),
$$

thus defining the functions $\left\{\vartheta^{(s)}(\mathbf{x})\right\}, s=e e$, oe, eo, oo. $\vartheta^{(e e)}(\mathbf{x})=\cos \left(2 \pi q_{1} x_{1}\right) \cos \left(2 \pi q_{2} x_{2}\right)$ is an even function in both $x_{1}$ and $x_{2}, \vartheta^{(o e)}(\mathbf{x})=\mathcal{H}_{1}\left\{\vartheta^{(e e)}\right\}(\mathbf{x}), \vartheta^{(e o)}(\mathbf{x})=\mathcal{H}_{2}\left\{\vartheta^{(e e)}\right\}(\mathbf{x})$, whilst $\vartheta^{(o o)}(\mathbf{x})=\mathcal{H}_{1}\left\{\mathcal{H}_{2}\left\{\vartheta^{(e e)}\right\}\right\}(\mathbf{x})$. To duplicate the structure of $\left\{\vartheta^{(\cdot)}(\mathbf{x})\right\}$, constructing a local QFT, a real-valued mother wavelet function $\psi^{(e e)}(\mathbf{x})$ is first chosen to be even in both $x_{1}$ and $x_{2}$, but not necessarily separable. We then define the hypercomplexing wavelet, by adding three extra functions to this function, where each extra function multiplies a unit quaternion, and where the functions are odd instead of even in either (or possibly both) of the two spatial directions.

Definition 3.2: Hypercomplexing wavelets.

The hypercomplexing wavelet function $\psi^{++}(\cdot)$ is formed from a 2-D real valued wavelet function $\psi^{(e e)}(\mathbf{x}) \in L^{2}(\mathbb{R})$, satisfying the admissibility condition of [55) and additionally the condition of:

$$
\psi^{(e e)}\left(x_{1}, x_{2}\right)=\psi^{(e e)}\left( \pm x_{1}, \pm x_{2}\right)=\psi^{(e e)}\left(x_{2}, x_{1}\right),
$$

i.e. the real wavelet is even in both $x_{1}$ and $x_{2}$ and symmetric across the indices. For such $\psi^{(e e)}(\mathbf{x})$ let: $\psi^{(o e)}(\mathbf{x})=$ $\mathcal{H}_{1}\left\{\psi^{(e e)}\right\}(\mathbf{x}), \psi^{(e o)}(\mathbf{x})=\mathcal{H}_{2}\left\{\psi^{(e e)}\right\}(\mathbf{x})$, and $\psi^{(o o)}(\mathbf{x})=\mathcal{H}_{2}\left\{\mathcal{H}_{1}\left\{\psi^{(e e)}\right\}\right\}(\mathbf{x})$, and form the hypercomplexing mother wavelet via

$$
\psi^{++}(\mathbf{x})=\widetilde{\mathcal{H C}} \psi^{(e e)}(\mathbf{x})=\psi^{(e e)}(\mathbf{x})+\boldsymbol{i} \psi^{(o e)}(\mathbf{x})+\boldsymbol{j} \psi^{(e o)}(\mathbf{x})-\boldsymbol{k} \psi^{(o o)}(\mathbf{x}) .
$$

The hypercomplexing wavelet is defined by a set of three functions constructed from the partial HTs of the even/even function in the two spatial directions, but note that $\mathcal{H C} \neq \widetilde{\mathcal{H C}}$, (where $\mathcal{H C}$ is given by equation 19.) The Fourier transforms of the functions forming $\psi^{++}(\mathbf{x})$ are given by:

$$
\begin{aligned}
& \Psi^{(o e)}(\mathbf{f})=(-\boldsymbol{j}) \operatorname{sgn}\left(f_{1}\right) \Psi^{(e e)}(\mathbf{f}), \quad \Psi^{(e o)}(\mathbf{f})=(-\boldsymbol{j}) \operatorname{sgn}\left(f_{2}\right) \Psi^{(e e)}(\mathbf{f}), \\
& \Psi^{(o o)}(\mathbf{f})=-\operatorname{sgn}\left(f_{1}\right) \operatorname{sgn}\left(f_{2}\right) \Psi^{(e e)}(\mathbf{f}) .
\end{aligned}
$$

$\Psi^{(e e)}(\mathbf{f}) \in \mathbb{R}$, as $\psi^{(e e)}(\mathbf{x})$ is even in both $x_{1}$ and $x_{2}$, and thus $\Psi_{Q}^{(e e)}(\mathbf{q}) \equiv \Psi^{(e e)}(\mathbf{q})$.

$$
\begin{aligned}
\Psi^{++*}(\mathbf{f}) & =\left(1-\boldsymbol{k} \operatorname{sgn}\left(f_{1}\right)+\operatorname{sgn}\left(f_{2}\right)+\boldsymbol{k} \operatorname{sgn}\left(f_{1}\right) \operatorname{sgn}\left(f_{2}\right)\right)^{*} \Psi^{(e e)}(\mathbf{f}) \\
& =\left(1+\boldsymbol{k} \operatorname{sgn}\left(f_{1}\right)+\operatorname{sgn}\left(f_{2}\right)-\boldsymbol{k} \operatorname{sgn}\left(f_{1}\right) \operatorname{sgn}\left(f_{2}\right)\right) \Psi^{(e e)}(\mathbf{f}) .
\end{aligned}
$$


The Fourier transform of the convolution of $\psi^{++*}(\mathbf{x})$ with $g(\mathbf{x}) \in L^{2}\left(\mathbb{R}^{2}\right)$, taking values in $\mathbb{R}$ is given by

$$
\mathcal{F}\left\{g * * \psi^{++*}\right\}(\mathbf{f})=G(\mathbf{f})\left(1+\boldsymbol{k} \operatorname{sgn}\left(f_{1}\right)+\operatorname{sgn}\left(f_{2}\right)-\boldsymbol{k} \operatorname{sgn}\left(f_{1}\right) \operatorname{sgn}\left(f_{2}\right)\right) \Psi^{(e e)}(\mathbf{f}) .
$$

Proposition 6: Admissibility of the hypercomplexing wavelets.

The quaternionic function $\psi^{++}(\mathbf{x})$, as well as any of its four component functions, all satisfy the admissibility condition.

Proof: As $\psi^{(e e)}(x)$ is an admissible wavelet function, it follows all its partial HTs are admissible, i.e. square integrable and satisfying the admissibility condition given by equation (55).

Theorem 2: Lack of hypercomplexity of $\psi^{++}(\mathbf{x})$.

The function $\psi^{++}(\mathbf{x})$ defined by equation (60) is not hypercomplex.

Proof: By direct calculation we note that the QFT of $\psi^{++}(\mathbf{x})$ takes the form

$$
\begin{aligned}
\mathcal{F}\left\{\psi^{++}\right\}(\mathbf{q}) & = \begin{cases}2 \Psi^{(e e)}(\mathbf{q}) & \text { if } q_{1}>0 \\
2 \operatorname{sgn}\left(q_{2}\right) \Psi^{(e e)}(\mathbf{q}) & \text { if } q_{1}<0\end{cases} \\
& \neq 0 \text { if } q_{1}<0 \quad \text { or } \quad q_{2}<0 .
\end{aligned}
$$

Thus the QFT of $\psi^{++}(\mathbf{x})$ is not wholly supported on positive quaternionic frequencies only, and $\psi^{++}(\mathbf{x})$ is $n o t$ a hypercomplex signal. The hypercomplexing wavelets are therefore denoted $\psi^{++}(\cdot)$, rather than $\psi^{(++)}(\cdot)$, but the wavelet coefficients they construct are $w_{\psi}^{(++)}(\boldsymbol{\xi} ; g)$, as they are hypercomplex signals in $\mathbf{b}$. Note that Chan $e t$ al. [2] define discrete quaternionic wavelet filters by combining two real-valued discrete wavelet functions that are approximately HT pairs, in a discrete version of equation (60). The objective of the quaternion-valued analysis is to form representations of signals that are hyperanalytic signals rather than using hyperanalytic signals in constructing the decomposition, and as we shall see, the hyperanalytizing wavelets are useful decomposition filters.

Proposition 7: FT and QFT of the members of the hypercomplexing wavelet family.

Any member of the hypercomplexing wavelet family defined by substituting in the form of the mother wavelet into equation 57 has a FT and a QFT given by

$$
\begin{aligned}
\Psi_{\boldsymbol{\xi}}^{++}(\mathbf{f})= & |a|\left[1+\operatorname{sgn}\left(\left[\boldsymbol{r}_{-\theta} \mathbf{f}\right]_{2}\right)-\boldsymbol{k} \operatorname{sgn}\left(\left[\boldsymbol{r}_{-\theta} \mathbf{f}\right]_{1}\right)\left[1-\operatorname{sgn}\left(\left[\boldsymbol{r}_{-\theta} \mathbf{f}\right]_{2}\right)\right]\right] \Psi^{(e e)}\left(a \boldsymbol{r}_{-\theta} \mathbf{f}\right) e^{-2 \pi j \mathbf{q}^{T} \mathbf{b}} \\
\Psi_{\boldsymbol{\xi}, Q}^{++}(\mathbf{q})= & |a| e^{-2 \pi \boldsymbol{i} q_{1} b_{1}} \mathcal{F}_{\mathcal{Q}}\left\{\psi_{(1, \theta, 0)}^{++}\right\}(a \mathbf{q}) e^{-2 \pi j q_{2} b_{2}} \\
= & |a| e^{-2 \pi i q_{1} b_{1}} \frac{1-\boldsymbol{k}}{2} \Psi^{(e e)}\left(a \boldsymbol{r}_{-\theta} \mathbf{q}\right)\left(1+\operatorname{sgn}\left(\left[\boldsymbol{r}_{-\theta} \mathbf{q}\right]_{1}\right)+\operatorname{sgn}\left(\left[\boldsymbol{r}_{-\theta} \mathbf{q}\right]_{2}\right)\right. \\
& \left.-\operatorname{sgn}\left(\left[\boldsymbol{r}_{-\theta} \mathbf{q}\right]_{1}\right) \operatorname{sgn}\left(\left[\boldsymbol{r}_{-\theta} \mathbf{q}\right]_{2}\right)\right)+\frac{1+\boldsymbol{k}}{2} \Psi^{(e e)}\left(a \boldsymbol{r}_{-\theta} \boldsymbol{J}_{\pi / 2} \mathbf{q}\right)\left(1+\operatorname{sgn}\left(\left[\boldsymbol{r}_{\theta} \mathbf{q}\right]_{1}\right)\right. \\
& \left.+\operatorname{sgn}\left(\left[\boldsymbol{r}_{\theta} \mathbf{q}\right]_{2}\right)-\operatorname{sgn}\left(\left[\boldsymbol{r}_{\theta} \mathbf{q}\right]_{1}\right) \operatorname{sgn}\left(\left[\boldsymbol{r}_{\theta} \mathbf{q}\right]_{2}\right)\right) e^{-2 \pi j q_{2} b_{2}}
\end{aligned}
$$

respectively.

Proof: The Fourier transform follows by direct calculation, whilst substituting in the form of the mother wavelet into equation (63) yields the expression for the QFT. Given the mother hypercomplexing wavelet was not a hypercomplex signal it is not surprising that neither will an arbitrary member of the family be a hypercomplex signal, as we may note from equation (64). We will build hypercomplexing wavelets from either $\psi^{(e e)}(\mathbf{x})=\psi_{S}^{(e e)}(\mathbf{x})$, the separable mother wavelet corresponding to a tensor product of two 1-D functions, or the isotropic real mother wavelet $\psi^{(e e)}(\mathbf{x})=\psi_{I}^{(e e)}(x)$, that is a function of $x=\sqrt{x_{1}^{2}+x_{2}^{2}}$. The reasoning behind these choices, is that we replace $\vartheta^{(e e)}(\mathbf{x})$ by functions that are even in both spatial directions, and that are chosen to satisfy equation (59). Either of $\psi_{I}^{++}(\mathbf{x})$ and $\psi_{S}^{++}(\mathbf{x})$ will enable us to decompose the observed image into local $\theta$-hypercomplex signals, for any given values of $a$ and $\theta$.

1) Separable hypercomplexing wavelets: The separable hypercomplexing wavelets are constructed from a single 1-D even function, denoted $\psi_{1 D}^{(e)}\left(x_{1}\right) \cdot \psi_{S}^{++}(\mathbf{x})$ is constructed to filter the signal to contributions corresponding to the same period in both spatial directions - by augmenting the doubly even function with the three extra wavelet functions we construct directional wavelets that are well localised in both direction and scale as well as spatial position. Using $\psi_{S}^{++}(\mathbf{x})$ we separate components according to their directional localisation, and the CWT needs to be calculate across the full range of $\theta \in[0, \pi / 2)$. First define the doubly even mother wavelet that will serve 

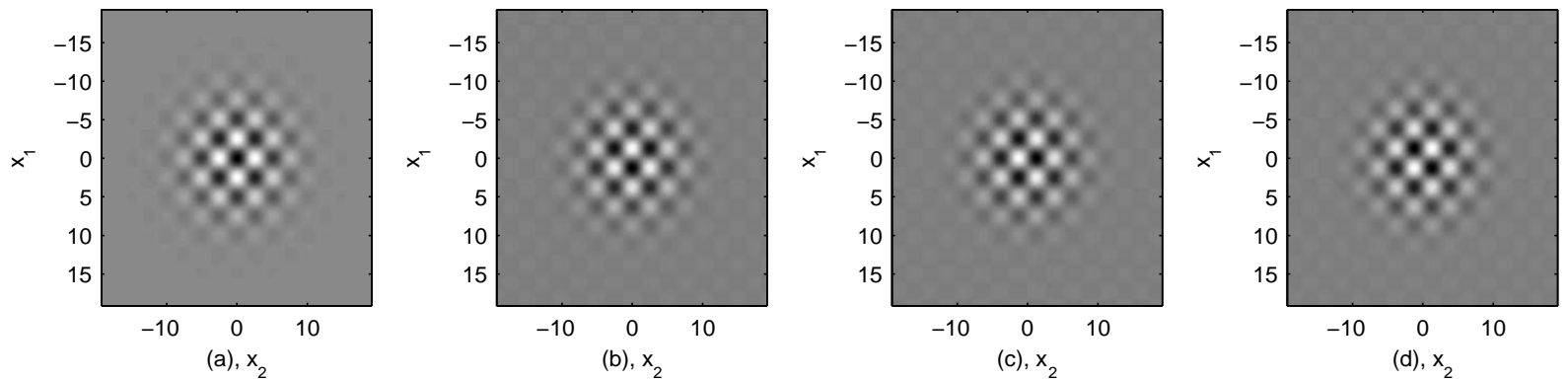

Fig. 1. The real part ((a)), first, second and third imaginary parts ((b), (c) and (d)) of the separable hypercomplexing Morse wavelets, with $n_{1}=n_{2}=0, \beta=9$ and $\gamma=4$.

as the real component of the quaternionic function $\psi^{++}(\mathbf{x})$, by $\psi^{(e e)}(\mathbf{x})=\psi_{1 D}^{(e)}\left(x_{1}\right) \psi_{1 D}^{(e)}\left(x_{2}\right)$. We define the 1-D function $\psi_{1 D}^{(o)}(x)=\mathcal{H}\left\{\psi_{1 D}^{(e)}\right\}(x)$. We may then observe that using equation 61 in this instance we find:

$$
\psi_{S}^{(o e)}(\mathbf{x})=\psi_{1 D}^{(o)}\left(x_{1}\right) \psi_{1 D}^{(e)}\left(x_{2}\right), \quad \psi_{S}^{(o e)}(\mathbf{x})=\psi_{1 D}^{(o)}\left(x_{1}\right) \psi_{1 D}^{(e)}\left(x_{2}\right), \quad \psi_{S}^{(o o)}(\mathbf{x})=\psi_{1 D}^{(o)}\left(x_{1}\right) \psi_{1 D}^{(o)}\left(x_{2}\right) .
$$

Assume $f_{0}=\arg _{f>0} \max \Psi_{1 D}^{(e)}(f)$. The wavelet functions may be considered a filtering the signal to frequencies near $f_{1}, f_{2} \approx \pm f_{0}$. Note that in 1-D analytic and anti-analytic wavelets are defined by combining $\psi_{1 D}^{(e)}\left(x_{1}\right)$ and $\psi_{1 D}^{(o)}\left(x_{1}\right)$ into a complex-valued wavelet function by:

$$
\psi_{1 D}^{( \pm)}\left(x_{1}\right)=\psi_{1 D}^{(e)}\left(x_{1}\right) \pm \boldsymbol{j} \psi_{1 D}^{(o)}\left(x_{1}\right) \quad \Psi_{1 D}^{( \pm)}\left(f_{1}\right)=\Psi_{1 D}^{(e)}\left(f_{1}\right) \pm \boldsymbol{j} \Psi_{1 D}^{(o)}\left(f_{1}\right) \in \mathbb{R} .
$$

Thus the FT, and the QFT of the members of the separable hypercomplexing wavelet family are given by:

$$
\begin{aligned}
\Psi_{S, \boldsymbol{\xi}}^{++}(\mathbf{f})= & |a|\left[\Psi^{(e)}\left(a\left[\boldsymbol{r}_{-\theta} \mathbf{f}\right]_{1}\right) \Psi^{(+)}\left(a\left[\boldsymbol{r}_{-\theta} \mathbf{f}\right]_{2}\right)+\boldsymbol{i} \Psi^{(o)}\left(a\left[\boldsymbol{r}_{-\theta} \mathbf{f}\right]_{1}\right) \Psi^{(-)}\left(a\left[\boldsymbol{r}_{-\theta} \mathbf{f}\right]_{2}\right)\right] e^{-2 \pi \mathbf{j}^{T} \mathbf{b},} \\
\Psi_{S, \boldsymbol{\xi} ; Q}^{++}(\mathbf{q})= & |a| e^{-2 \pi i q_{1} b_{1}} \frac{1-\boldsymbol{k}}{2}\left(\Psi^{(+)}\left(a\left[\boldsymbol{r}_{-\theta} \mathbf{q}\right]_{1}\right) \Psi^{(e)}\left(a\left[\boldsymbol{r}_{-\theta} \mathbf{q}\right]_{2}\right)+\Psi^{(-)}\left(a\left[\boldsymbol{r}_{-\theta} \mathbf{q}\right]_{1}\right) \Psi^{(o)}\left(a\left[\boldsymbol{r}_{-\theta} \mathbf{q}\right]_{2}\right)\right) \\
& +\frac{1+\boldsymbol{k}}{2}\left(\Psi^{(+)}\left(a\left[\boldsymbol{r}_{-\theta} \boldsymbol{J}_{\pi / 2} \mathbf{q}\right]_{1}\right) \Psi^{(e)}\left(a\left[\boldsymbol{r}_{-\theta} \boldsymbol{J}_{\pi / 2} \mathbf{q}\right]_{2}\right)\right. \\
& \left.+\Psi^{(-)}\left(a\left[\boldsymbol{r}_{-\theta} \boldsymbol{J}_{\pi / 2} \mathbf{q}\right]_{1}\right) \Psi^{(o)}\left(a\left[\boldsymbol{r}_{-\theta} \boldsymbol{J}_{\pi / 2} \mathbf{q}\right]_{2}\right)\right) e^{-2 \pi j q_{2} b_{2}},
\end{aligned}
$$

respectively. The interpretation of $\Psi_{S, \boldsymbol{\xi}}^{++}(\mathbf{f})$ is that the hypercomplexing wavelets are naturally treated in a rotated frame of reference, whilst from the form of the QFT it is apparent that there is no choice of rotation that will make the wavelet $\theta$-hypercomplex. However, as the mother failed to be hypercomplex, this is not surprising.

Using the separable hypercomplexing wavelets each of the two analytic wavelet functions may be given a polar representation, i.e.

$\psi_{1 D}^{(+)}\left(x_{1}\right)=\left|\psi_{1 D}^{(+)}\left(x_{1}\right)\right| e^{2 \pi \boldsymbol{j} \phi_{\psi, 1 D}^{(+)}(x)}$, and thus it transpires that $\psi_{S}^{++}(\mathbf{x})=\left|\psi_{1 D}^{(+)}\left(x_{1}\right)\right|\left|\psi_{1 D}^{(+)}\left(x_{2}\right)\right| e^{2 \pi \boldsymbol{i} \phi_{\psi, 1 D}^{(+)}\left(x_{1}\right)}$

$e^{2 \pi \boldsymbol{k} 0} e^{2 \pi \boldsymbol{j} \phi_{\psi, 1 D}^{(+)}\left(x_{2}\right)}$ or $\psi_{S}^{++}(\mathbf{x})=\left|\psi_{S}^{++}(\mathbf{x})\right| e^{2 \pi \boldsymbol{i} \alpha_{\psi}(\mathbf{x})} e^{2 \pi \boldsymbol{k} \gamma_{\psi}(\mathbf{x})} e^{2 \pi \boldsymbol{j} \beta_{\psi}(\mathbf{x})}$, with $\left|\psi_{S}^{++}(\mathbf{x})\right|=\left|\psi_{1 D}^{(+)}\left(x_{1}\right)\right|\left|\psi_{1 D}^{(+)}\left(x_{2}\right)\right|$, $\alpha_{\psi}(\mathbf{x})=\phi_{\psi, 1 D}^{(+)}\left(x_{1}\right), \beta_{\psi}(\mathbf{x})=\phi_{\psi, 1 D}^{(+)}\left(x_{2}\right)$, and $\gamma_{\psi}(\mathbf{x})=0$. The separable hypercomplexing wavelets are representing local structure by finding the local structural information in $x_{1}$ and $x_{2}$ separately, and representing this in terms of a magnitude and two phases. $\phi_{\psi, 1 D}^{(+)}(x)$ is the local frequency content of the 1-D function, $\psi_{1 D}^{(+)}\left(x_{1}\right)$, and in the two separate spatial directions the same frequency behaviour is considered. This is obvious from from Figure 1 where the wavelets correspond to two separable oscillations local to $\mathrm{x}=\mathbf{0}$, with the same local period in both spatial directions.

2) Isotropic hypercomplexing wavelets : Basing the hypercomplexing wavelet on an isotropic mother wavelet, $\psi_{I}^{(e e)}(\mathbf{x})$, wavelet coefficients are produced by filtering the signal to retain contributions with the same period. Using the extra components potentially separable structures can be characterised that have the same period present at a given spatial location. Figure 2 shows isotropic hypercomplexing wavelets, and how the spatial localisation is 
radial, whilst each added component is odd in $x_{1}$ and $x_{2}$, respectively. When an isotropic mother wavelet is used then we obtain that:

$$
\psi_{I}^{(o e)}(\mathbf{x})=\mathcal{H}_{1}\left\{\psi_{I}^{(e e)}\right\}(\mathbf{x}), \quad \psi_{I}^{(e o)}(\mathbf{x})=\mathcal{H}_{2}\left\{\psi_{I}^{(e e)}\right\}(\mathbf{x}), \quad \psi_{I}^{(o o)}(\mathbf{x})=\mathcal{H}_{T}\left\{\psi_{I}^{(e e)}\right\}(\mathbf{x}) .
$$

These wavelets are able to determine any preference for variation in the direction $\chi=\tan ^{-1}\left(x_{2} / x_{1}\right)=0, \chi=\pi / 2$ and $\chi=\pi / 4$, respectively. This is clear from the example of an isotropic hypercomplexing wavelet given in Figure 2

3) Hypercomplex wavelets : Following work on discrete wavelet filters given in [1][p. 138] a real directional wavelet based on the 1-D analytic continuous wavelet filters of section $\amalg$ II-A.1 will be constructed via first defining the $\pi / 4$ directional wavelet function:

$$
\psi_{D}^{(\pi / 4)}(\mathbf{x})=\psi_{1 D}^{(e)}\left(x_{1}\right) \psi_{1 D}^{(e)}\left(x_{2}\right)-\psi_{1 D}^{(o)}\left(x_{1}\right) \psi_{1 D}^{(o)}\left(x_{2}\right) .
$$

$\psi_{D}^{(\pi / 4)}(\mathbf{x})$ is local in frequency to $\mathbf{f}= \pm \sqrt{2} f_{0}[\cos (\pi / 4) \sin (\pi / 4)]^{T}$. Formally, the above wavelet, does not satisfy (59), and cannot determine existing separable structure as it only considers structure along the diagonal direction and not variation perpendicular to this direction. To align the wavelet with the axis of observation we define

$$
\psi_{D}(\mathbf{x})=\psi_{D}^{(\pi / 4)}\left(\boldsymbol{r}_{\pi / 4} \mathbf{x}\right)=\psi_{1 D}^{(e)}\left(\frac{x_{1}-x_{2}}{\sqrt{2}}\right) \psi_{1 D}^{(e)}\left(\frac{x_{1}+x_{2}}{\sqrt{2}}\right)-\psi_{1 D}^{(o)}\left(\frac{x_{1}-x_{2}}{\sqrt{2}}\right) \psi_{1 D}^{(o)}\left(\frac{x_{1}+x_{2}}{\sqrt{2}}\right) .
$$

Thus $\Psi_{D}(\mathbf{x})$ will be local in frequency to $\mathbf{f}= \pm \sqrt{2} f_{0}\left[\begin{array}{ll}1 & 0\end{array}\right]^{T}$. The function is extended to a quaternionic object by calculating its total and partial HTs:

$$
\begin{aligned}
& \mathcal{H}_{1}\left\{\psi_{D}\right\}(\mathbf{x})=\psi_{1 D}^{(o)}\left(\frac{x_{1}-x_{2}}{\sqrt{2}}\right) \psi_{1 D}^{(e)}\left(\frac{x_{1}+x_{2}}{\sqrt{2}}\right)-\psi_{1 D}^{(e)}\left(\frac{x_{1}-x_{2}}{\sqrt{2}}\right) \psi_{1 D}^{(o)}\left(\frac{x_{1}+x_{2}}{\sqrt{2}}\right)=\psi_{D, 2}(\mathbf{x}), \\
& \mathcal{H}_{2}\left\{\psi_{D}\right\}(\mathbf{x})=0, \quad \mathcal{H}_{T}\left\{\psi_{D}\right\}(\mathbf{x})=0 .
\end{aligned}
$$

Formally by direct calculation: $\psi_{D}^{(++)}(\mathbf{x})=\psi_{D}(\mathbf{x})+\boldsymbol{i} \psi_{D, 2}(\mathbf{x}) \cdot \psi_{D}^{(++)}(\mathbf{x})$ is a directional quaternionic wavelet function, naturally taking the form of a wavelet suggested by [1]. This wavelet is formed by a similar set of operations to the hypercomplexing wavelet but we have given it the notation $\psi_{D}^{(++)}(\mathbf{x})$, as formally, it is also a hypercomplex signal. As the third and fourth real-valued components of the quaternionic objects are equivalently zero, and it is the non-zero fourth component, which prevents the hypercomplexing wavelet from being a hypercomplex signal. $\psi_{D}^{(++)}(\mathbf{x})$ can be treated as a complex-valued wavelet (see footnote [2), but is not equivalent to the complex directional cone wavelets of Antoine et al. [21]. The two components of $\psi_{D}^{(++)}(\mathbf{x})$ are plotted in Figure 4

\section{B. Monogenic wavelets}

The hypercomplexing wavelets were constructed so they filter the image as a local version of the QFT in orientation $\theta$, and their usage will (as we shall see) produce wavelet coefficients that are $\theta$-hypercomplex signals. The monogenic signal is locally identifying a dominant orientation of variations and constructing, by adding the Riesz transforms of the components to the signal, a polar representation of such variation. The monogenic signal of a globally unidirectional signal could be represented as an Inverse UQFT (IUQFT) in $\boldsymbol{e}_{\nu}$, constructed only from the positive frequencies of the UQFT in $e_{\nu}$, and had an interpretation from equation (18). The direction of a unidirectional signal could be determined directly by the ratio of the Riesz transform components. A scale-localised version of this construction would correspond to filtering the image in space and spatial frequency, thus isolating structure with a given period and spatial location, and then constructing the Riesz transforms of the local component. The resulting quaternionic function could be represented as a quaternionic object. The route to constructing the scale localised monogenic signal in one step will be using quaternionic mother wavelet functions.

Definition 3.3: Monogenic wavelets.

The monogenic extension of any given real-valued mother wavelet $\psi^{(r)}(\mathbf{x})$ is given by

$$
\psi^{(+)}(\mathbf{x})=\mathcal{M} \psi^{(r)}(\mathbf{x})=\psi^{(r)}(\mathbf{x})+\mathcal{R} \psi^{(r)}(\mathbf{x})=\psi^{(r)}(\mathbf{x})+\left[\boldsymbol{i} \psi_{R}^{(1)}(\mathbf{x})+\boldsymbol{j} \psi_{R}^{(2)}(\mathbf{x})\right],
$$

where $\psi_{R}^{(1)}=\mathcal{R}_{1} \psi^{(r)}, \psi_{R}^{(2)}=\mathcal{R}_{2} \psi^{(r)}$. 
Note that the terminology 'monogenic wavelet' has been utilized before in a different context: see work by Cerejeiras et al. [70]. The monogenic wavelets of [70] are defined on the unit ball and based on Möbius transformations, and are distinct from our wavelets.

Lemma 1: Properties of the wavelet Riesz components.

Each component of the Riesz transform of a wavelet is also a wavelet.

Proof: As

$$
\int_{E_{2}} \psi_{R}^{(s) 2}(\mathbf{x}) d^{2} \mathbf{x}=\int_{E_{2}}\left|\Psi_{R}^{(s)}(\mathbf{f})\right|^{2} d^{2} \mathbf{f} \leq \int_{E_{2}}\left|\Psi^{(e)}(\mathbf{f})\right|^{2} d^{2} \mathbf{f}<\infty, s=1,2,
$$

square integrability follows as the wavelets were constructed from a real mother wavelet. Relations (41), (42), hold of course, for $\psi^{(+)}$and its components $\psi^{(r)}, \psi_{R}^{(1)}$, and $\psi_{R}^{(2)}$. Denote by $c_{\psi^{(s)}}$ the value of equation (55) with $\psi_{R}^{(s)}$. Equation (41) in combination with the fact that $\cos ^{2} \phi$ and $\sin ^{2} \phi$ are always between 0 and 1, imply that $0<c_{\psi^{(1)}} \leq c_{\psi^{(r)}}<\infty$ and $0<c_{\psi^{(2)}} \leq c_{\psi^{(r)}}<\infty$. Consequently $\psi_{R}^{(1)}$ and $\psi_{R}^{(2)}$ satisfy the admissibility condition, (55), and can be considered as wavelets. The lemma implies that by the monogenic extension of the real wavelet we have constructed two additional well-behaved functions and we may then consider the properties of the monogenic extension as a whole.

Proposition 8: Admissibility of the monogenic mother wavelet.

The monogenic extension of a real wavelet is also a wavelet.

Proof: It is obvious from (70) and (42) that $\left|\Psi^{(+)}(\mathbf{f})\right|^{2}=\left|\Psi^{(r)}(\mathbf{f})\right|^{2}+\left|\Psi_{R}^{(1)}(\mathbf{f})\right|^{2}+\left|\Psi_{R}^{(2)}(\mathbf{f})\right|^{2}+2 \sin \phi\left|\Psi^{(r)}(\mathbf{f})\right|^{2}$. Integrating over all frequencies as in (55) and making use of the Hermitian property of the FT of a real image we find that $c_{\psi^{(+)}}=c_{\psi^{(r)}}+c_{\psi^{(1)}}+c_{\psi^{(2)}}$. Therefore, since each of $\psi^{(r)}, \psi_{R}^{(1)}$, and $\psi_{R}^{(2)}$ satisfy the admissibility condition, $\psi^{(+)}$also satisfies the admissibility condition and can be considered as a monogenic wavelet. Moreover, $c_{\psi^{(1)}}+c_{\psi^{(2)}}=c_{\psi^{(r)}}$, so $c_{\psi^{(+)}}=2 c_{\psi^{(r)}}$ also holds. Hence the monogenic extension of the wavelet is a valid analysis wavelet and we may consider its further properties.

The monogenic wavelets may be represented in polar form via its modulus, orientation, and phase respectively given by:

$$
\begin{aligned}
\left|\psi_{\boldsymbol{\xi}}^{(+)}(\mathbf{x})\right| & =\sqrt{\left[\psi_{\boldsymbol{\xi}}^{(r)}(\mathbf{x})\right]^{2}+\left[\psi_{R, \boldsymbol{\xi}}^{(1)}(\mathbf{x})\right]^{2}+\left[\psi_{R, \boldsymbol{\xi}}^{(2)}(\mathbf{x})\right]^{2}}, \\
\boldsymbol{e}_{\nu_{\boldsymbol{\xi}}}(\mathbf{x}) & =\left[\boldsymbol{i} \frac{\psi_{R, \boldsymbol{\xi}}^{(1)}(\mathbf{x})}{\sqrt{\left[\psi_{R, \boldsymbol{\xi}}^{(1)}(\mathbf{x})\right]^{2}+\left[\psi_{R, \boldsymbol{\xi}}^{(2)}(\mathbf{x})\right]^{2}}}+\boldsymbol{j} \frac{\psi_{R, \boldsymbol{\xi}}^{(2)}(\mathbf{x})}{\left[\psi_{R, \boldsymbol{\xi}}^{(1)}(\mathbf{x})\right]^{2}+\left[\psi_{R, \boldsymbol{\xi}}^{(2)}(\mathbf{x})\right]^{2}}\right] \\
& =\boldsymbol{i} \cos \left(\nu_{\xi}(\mathbf{x})\right)+\boldsymbol{j} \sin \left(\nu_{\xi}(\mathbf{x})\right), \\
\phi_{\boldsymbol{\xi}}(\mathbf{x}) & =\tan ^{-1}\left(\operatorname{sgn}\left[\psi_{R, \boldsymbol{\xi}}^{(2)}(\mathbf{x})\right] \frac{\sqrt{\left[\psi_{R, \boldsymbol{\xi}}^{(1)}(\mathbf{x})\right]^{2}+\left[\psi_{R, \boldsymbol{\xi}}^{(2)}(\mathbf{x})\right]^{2}}}{\psi_{\boldsymbol{\xi}}^{(r)}(\mathbf{x})}\right)
\end{aligned}
$$

The orientation is thus given an angular representation. The wavelet is a function with spatial energy given by the modulus, has a local zero-crossing structure given by $\phi_{\psi_{\xi}}(\mathbf{x})$, and is showing a local orientation preference to angle $\nu_{\psi_{\xi}}(\mathbf{x})$. The CWT is decomposing the image in terms of localised oscillations with a local period determined from $\phi_{\psi_{\xi}}(\mathbf{x})$. For a more thorough discussion of the interpretation of the polar representation of $\psi_{\boldsymbol{\xi}}^{(+)}(\mathbf{x})$, see [71].

Proposition 9: FT and QFT of the members monogenic wavelet family. The FT and QFT of the translated, dilated, and rotated monogenic wavelet is

$$
\begin{gathered}
\Psi_{\boldsymbol{\xi}}^{(+)}(\mathbf{f})=\mathcal{F} \psi_{\xi}^{(+)}(\mathbf{x})=\Psi_{\boldsymbol{\xi}}^{(r)}(\mathbf{f})+\left[\boldsymbol{i} \Psi_{R, \boldsymbol{\xi}}^{(1)}(\mathbf{f})+\boldsymbol{j} \Psi_{R, \boldsymbol{\xi}}^{(2)}(\mathbf{f})\right] \\
=\{1+[-\boldsymbol{k} \cos (\phi-\theta)+\sin (\phi-\theta)]\} \Psi_{\boldsymbol{\xi}}^{(r)}(\mathbf{f}) . \\
\Psi_{\boldsymbol{\xi} ; Q}^{(+)}(\mathbf{q})=\mathcal{F}_{\mathcal{Q}} \psi_{\boldsymbol{\xi}}^{(+)}(\mathbf{x})=\Psi_{\boldsymbol{\xi} ; Q}^{(r)}(\mathbf{q})+\left[\boldsymbol{i} \Psi_{R, \boldsymbol{\xi} ; Q}^{(1)}(\mathbf{q})+\Psi_{R, \boldsymbol{\xi} ; Q}^{(2)}(\mathbf{q}) \boldsymbol{j}\right] \\
=\frac{a}{2} e^{-2 \boldsymbol{i} \pi q_{1} b_{1}}\left[[1+\cos (\eta-\theta)+\sin (\eta-\theta)]\left[\Psi_{Q}^{(r)}\left(\boldsymbol{r}_{-\theta} \mathbf{q}\right)-\boldsymbol{k} \Psi_{Q}^{(r)}\left(\boldsymbol{J}_{\pi / 2+\theta / 2} \mathbf{q}\right)\right]\right. \\
\left.+[1+\cos (\eta+\theta)+\sin (\eta+\theta)]\left[\Psi_{Q}^{(r)}\left(\boldsymbol{r}_{\theta} \mathbf{q}\right)+\boldsymbol{k} \Psi_{Q}^{(r)}\left(\boldsymbol{J}_{\pi / 2-\theta / 2} \mathbf{q}\right)\right]\right] e^{-2 \boldsymbol{j} \pi q_{2} b_{2}} .
\end{gathered}
$$


Proof: See section [I-A As noted by Holschneider [32] the entire family of wavelets constructed from a 1-D analytic mother wavelet are analytic signals. Analogously, for the monogenic wavelets, we may state the result:

Theorem 3: Monogenicity of the members of the wavelet family.

The members of the family of equation (57) generated from the monogenic wavelet $\psi^{(+)}(\cdot)$ through $U_{\boldsymbol{\xi}}$ are also $\theta$-monogenic signals, this should be contrasted with the $\theta$-hypercomplexing wavelets, that are not $\theta$-hypercomplex signals.

Proof: This is not a trivial result as we noted in equation (44), and corresponds to more than just a simple 2-D extension of the 1-D result. An arbitrary member of the wavelet family may be recast as $U_{\boldsymbol{\xi}} \psi^{(r)}(\mathbf{x})+$ $\boldsymbol{i} U_{\boldsymbol{\xi}} \mathcal{R}_{1} \psi^{(r)}(\mathbf{x})+\boldsymbol{j} U_{\boldsymbol{\xi}} \mathcal{R}_{2} \psi^{(r)}(\mathbf{x})$ as the Riesz kernels commute with dilations and translations, $\left[\mathcal{D}_{a}, \mathcal{R}_{j}\right]=0$, $\left[\mathcal{T}_{\mathbf{b}}, \mathcal{R}_{j}\right]=0$. Of course $R_{\theta} \psi^{(r)}(\mathbf{x})$ is a $\theta$-monogenic signal/function from equation [40), and thus from proposition 11. we may note the result. Denote by $\psi^{(e)}(x)$ an isotropic real mother wavelet function.

1) Isotropic monogenic wavelets:

Proposition 10: The wavelet family of the isotropic monogenic mother wavelet. If $\psi^{(r)}(\mathbf{x})=\psi^{(e)}(x)$, then any member of the monogenic wavelet family in the Fourier and Quaternionic Fourier domain have a simplified form with $\boldsymbol{\xi}_{0}=[a, 0 ; \mathbf{b}]^{T}$ by:

$$
\begin{aligned}
\psi_{\boldsymbol{\xi}}^{(+)}(\mathbf{x}) & =\psi_{\boldsymbol{\xi}_{0}}^{(e)}(\mathbf{x})+\boldsymbol{i}\left[\cos (\theta) \psi_{R, \boldsymbol{\xi}_{0}}^{(1)}(\mathbf{x})+\sin (\theta) \psi_{R, \boldsymbol{\xi}_{0}}^{(2)}(\mathbf{x})\right]+\boldsymbol{j}\left[-\sin (\theta) \psi_{R, \boldsymbol{\xi}_{0}}^{(1)}(\mathbf{x})+\cos (\theta) \psi_{\boldsymbol{\xi}_{0}}^{(2)}(\mathbf{x})\right] \\
\Psi_{\boldsymbol{\xi}}^{(+)}(\mathbf{f}) & =\{1+[-\boldsymbol{k} \cos (\phi-\theta)+\sin (\phi-\theta)]\} a \Psi^{(e)}(a f) e^{-j 2 \pi \mathbf{f b}}, \\
\Psi_{\boldsymbol{\xi} ; Q}^{(+)}(\mathbf{q}) & =e^{-2 \pi \boldsymbol{i} q_{1} b_{1}} a \Psi^{(e)}(a q)[1+\cos (\theta)(\cos (\eta)+\sin (\eta))+\boldsymbol{k} \sin (\theta)(-\sin (\eta)+\cos (\eta))] e^{-2 \pi \boldsymbol{j} q_{2} b_{2}} .
\end{aligned}
$$

Proof: See section [-B As an example of the isotropic monogenic wavelet, see Figure 3 The isotropic structure of the magnitude is clear, whilst variations over the $x_{1}$ and $x_{2}$ directions may respectively be determined.

Proposition 11: $\theta=\pi$ Equivalence to the anti-monogenic wavelet.

When the monogenic mother has a radially symmetric real part, then the $\boldsymbol{\xi}_{\pi}$ monogenic wavelet coincides with the $\boldsymbol{\xi}$ anti-monogenic wavelet, and with $\boldsymbol{\xi}_{\pi}=[a, \theta+\pi ; \mathbf{b}]^{T}$,

$$
\psi_{\boldsymbol{\xi}_{\pi}}^{(+)}(\mathbf{x})=\psi_{\boldsymbol{\xi}}^{(-)}(\mathbf{x}) .
$$

Proof: This we can see by replacing $\theta$ by $\theta+\pi$ in (76).

2) Directional monogenic wavelets : The isotropic monogenic wavelets will localise an image to a particular scale at each spatial position, and then represent the structure present at that scale and position by a plane wave. In some applications it may be suitable to localise in both scale and orientation, as several components are present at each position and at the same scale in different directions, and we start by constructing a quaternionic wavelet starting with a real directional wavelet defined by equation [69. The Riesz transforms are found in Appendix 【-C and we obtain:

$$
\begin{aligned}
& \mathcal{R}_{1}\left\{\psi_{D}\right\}(\mathbf{x})=4 \int_{0}^{\infty} \int_{0}^{\infty} \frac{\sqrt{2} g_{1}}{g}\left[\Psi^{(e)}\left(g_{1}\right) \Psi^{(e)}\left(g_{2}\right)\right] \sin \left(2 \pi x_{1}\left(\frac{g_{2}+g_{1}}{\sqrt{2}}\right)\right) d^{2} \mathbf{g}=\psi_{D, 3}(\mathbf{x}) \\
& \mathcal{R}_{2}\left\{\psi_{D}\right\}(\mathbf{x})=0 .
\end{aligned}
$$

The complex directional wavelet (see footnote 2 on treating $\psi_{D}^{(+)}(\mathbf{x})$ as complex-valued) is formed by: $\psi_{D}^{(+)}(\mathbf{x})=$ $\psi_{D}(\mathbf{x})+\boldsymbol{i} \psi_{D, 3}(\mathbf{x}) \cdot \psi_{D}^{(+)}(\mathbf{x})$ is a directional monogenic signal. $\psi_{D}^{(+)}(\mathbf{x})$ 's directional structure may clearly be observed from a contour plot of its two components in Figure $4 \psi_{D}^{(+)}(\mathbf{x})$ similar to the complex directional wavelet of [1], but is not equivalent to $\psi_{D}^{(++)}(\mathbf{x})$, defined in section III-A.3 When the Riesz component is constructed from $\psi_{D}(\mathbf{x})$, the spatial frequency modulation to $\Psi_{D}(\mathbf{f})$ is $f_{1} / f$ rather than $\operatorname{sgn}\left(f_{1}\right)$. Of course $\Psi_{D}(\mathbf{f})$ is mainly limited in frequency to $\mathbf{f}=\left[\begin{array}{ll} \pm \sqrt{2} f_{0}, & 0\end{array}\right]^{T}$, and for $\mathbf{f}=\left[\begin{array}{ll} \pm \sqrt{2} f_{0}+\delta f_{1}, & \delta f_{2}\end{array}\right]^{T}, f_{1} / f= \pm 1+\delta f_{1} / f_{0}^{2}+o\left(\delta f_{i}^{2}\right)$, and so up to a small corrective error term $\psi_{D, 2}(\mathbf{x})=\psi_{D, 3}(\mathbf{x})$. This is verified by the visual similarity of Figures (4) (b) and (c). The advantage of using a second component constructed from the Riesz transforms, i.e. using $\psi_{D, 3}(\mathbf{x})$ rather than using $\psi_{D, 2}(\mathbf{x})$, the hypercomplex wavelet second component, is that the quaternionic mother wavelet when rotated satisfies the relationships given by equation (44). The complex function is then a bona-fide monogenic function coupled with a single quaternionic conjugate, an anti-monogenic function. 

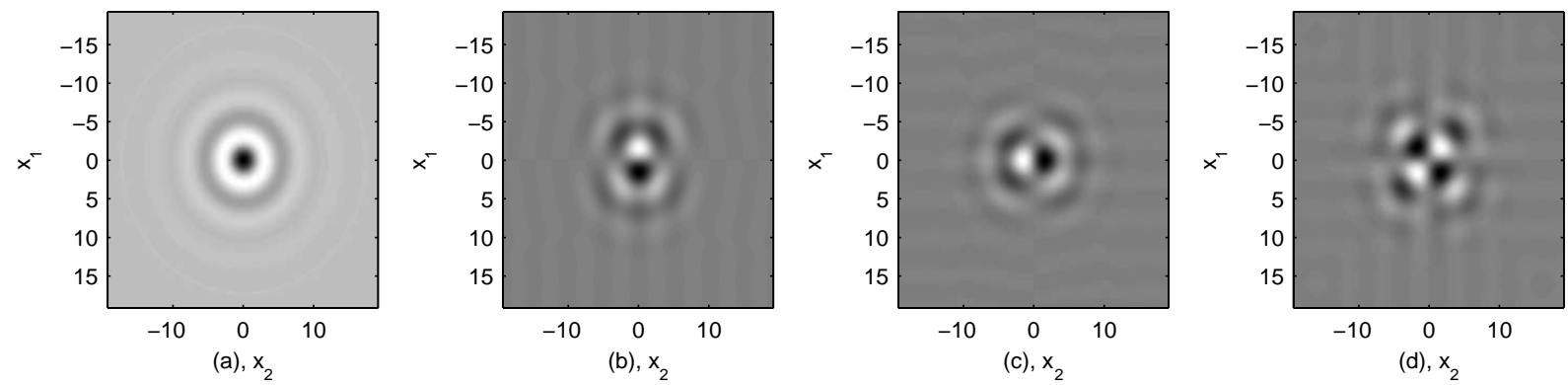

Fig. 2. The real part (a), first, second and third imaginary parts, (b), (c) and (d), of the isotropic hypercomplexing Morse wavelets, with $n=0, l=9$ and $m=4$.

\section{Quaternionic Morse wavelets}

The 1-D Morse wavelets were introduced by Daubechies and Paul [72], [73] and applied in signal processing by Bayram and Baraniuk, as well as Olhede \& Walden [33], [74]. In 1-D, according to [33], the analytic [ $\beta, \gamma, n]$ Morse wavelet is $\psi_{1 D, n ; \beta, \gamma}^{(+)}(\cdot)=\psi_{1 D, n ; \beta, \gamma}^{(e)}(\cdot)+\boldsymbol{j} \psi_{1 D, n ; \beta, \gamma}^{(o)}(\cdot)$, and is defined in the frequency domain $(c f$ [33, p. 2666]) by

$$
\Psi_{1 D, n ; \beta, \gamma}^{(+)}(f)=\sqrt{2} A_{n ; \beta, \gamma}(2 \pi f)^{\beta} e^{-(2 \pi f)^{\gamma}} L_{n}^{c}\left[2(2 \pi f)^{\gamma}\right], f>0 .
$$

For $f<0, \Psi_{1 D, n ; \beta, \gamma}^{(+)}(f)$ vanishes. We use the notation $c=(2 \beta+1) / \gamma-1$, where $\beta, \gamma, \in \mathbb{R}^{+}$. Moreover, $A_{n ; \beta, \gamma}=\sqrt{\pi \gamma 2^{c+1} \Gamma(1+n) / \Gamma(n+c+1)}$ is a normalisation constant and $\mathrm{L}_{n}^{c}(x)$ is the generalised Laguerre polynomial which can be written in a series form:

$$
\mathrm{L}_{n}^{c}(x)=\sum_{r=0}^{n}(-1)^{r} \frac{\Gamma(1+n+c)}{\Gamma(1+c+r) \Gamma(1+n-r)} \frac{x^{r}}{r !} .
$$

Metikas and Olhede [44] defined isotropic real Morse wavelets, augmented into quaternionic functions, using the Riesz transforms. The work in [44] determined specific families of isotropic wavelets that could be considered optimally local to a given radial domain, as well as performed estimation of local image characteristics using what in this article would be denoted isotropic monogenic wavelets. The $n$-th order real isotropic Morse wavelet is a radially symmetric function, $\psi_{n ; l, m}^{(e)}(\cdot)$, where $l=\beta+1 / 2$, and $m=\gamma$. Its FT and QFT are given by, [44],

$$
\Psi_{n ; l, m}^{(e)}(f) \equiv \Psi_{n ; l, m, Q}^{(e)}(f)=A_{n ; l, m}^{\prime}(2 \pi f)^{l} e^{-(2 \pi f)^{m}} \mathrm{~L}_{n}^{c^{\prime}}\left[2(2 \pi f)^{m}\right]
$$

where $c^{\prime}=(2 l+2) / m-1, m \geq 1, l>0, l \geq m / 2-1$, and $A_{n ; l, m}^{\prime}=\sqrt{m 2^{c^{\prime}+1} \Gamma(1+n) / \Gamma\left(n+c^{\prime}+1\right)}$. The first two requirements are necessary to make $\psi_{n ; l, m}^{(e)}(f)$ a wavelet, and the second constraint ensures the wavelet is related to a suitable localization operator [44]. The 2-D generalization of the analytic Morse wavelets is approached by the following method: we first combine the even 1-D Morse wavelets in a suitable tensor product, or we use isotropic real wavelet functions as $\psi^{(r)}(\mathbf{x})$. We then, to mimic the analytic wavelets, augment the real mother wavelet by two or three wavelet functions and form a quaternionic wavelet function, by $\mathcal{W} \psi^{(r)}(\mathbf{x})$.

Definition 3.4: Separable hypercomplexing Morse wavelets.

The $\beta$, and $\gamma$, separable hypercomplexing Morse wavelets are for any $\left(n_{1}, n_{2}\right) \in \mathbb{N}^{2}$ defined by

$$
\begin{aligned}
\psi_{S, \boldsymbol{n} ; \beta, \gamma}^{++}(\mathbf{x})= & \psi_{1 D, n_{1} ; \beta, \gamma}^{(e)}\left(x_{1}\right) \psi_{1 D, n_{2} ; \beta, \gamma}^{(e)}\left(x_{2}\right)+\boldsymbol{i} \psi_{1 D, n_{1} ; \beta, \gamma}^{(o)}\left(x_{1}\right) \psi_{1 D, n_{2} ; \beta, \gamma}^{(e)}\left(x_{2}\right) \\
& +\boldsymbol{j} \psi_{1 D, n_{1} ; \beta, \gamma}^{(e)}\left(x_{1}\right) \psi_{1 D, n_{2} ; \beta, \gamma}^{(o)}\left(x_{2}\right)-\boldsymbol{k} \psi_{1 D, n_{1} ; \beta, \gamma}^{(o)}\left(x_{1}\right) \psi_{1 D, n_{2} ; \beta, \gamma}^{(o)}\left(x_{2}\right) .
\end{aligned}
$$

For a plot of the real-valued components of a given separable quaternionic hypercomplexing Morse wavelet see Figures 1 (a), (b), (c) and (d). The separable hypercomplexing Morse wavelets exhibit the chequer-board patterns of separable wavelets, and can, once the CWT has been calculated, be used to find local separable structure in an image at a given orientation.

Definition 3.5: Isotropic hypercomplexing Morse wavelets.

The $l$, and $m$, isotropic hypercomplexing Morse wavelets are for $n \in \mathbb{N}$, defined by

$$
\psi_{I, \boldsymbol{n} ; l, m}^{++}(\mathbf{x})=\psi_{n ; l, m}^{(e)}(\mathbf{x})+\boldsymbol{i} \mathcal{H}_{1}\left\{\psi_{n ; l, m}^{(e)}\right\}(\mathbf{x})+\boldsymbol{j} \mathcal{H}_{2}\left\{\psi_{n ; l, m}^{(e)}\right\}(\mathbf{x})-\boldsymbol{k} \mathcal{H}_{2} \mathcal{H}_{1}\left\{\psi_{n ; l, m}^{(e)}\right\}(\mathbf{x})
$$



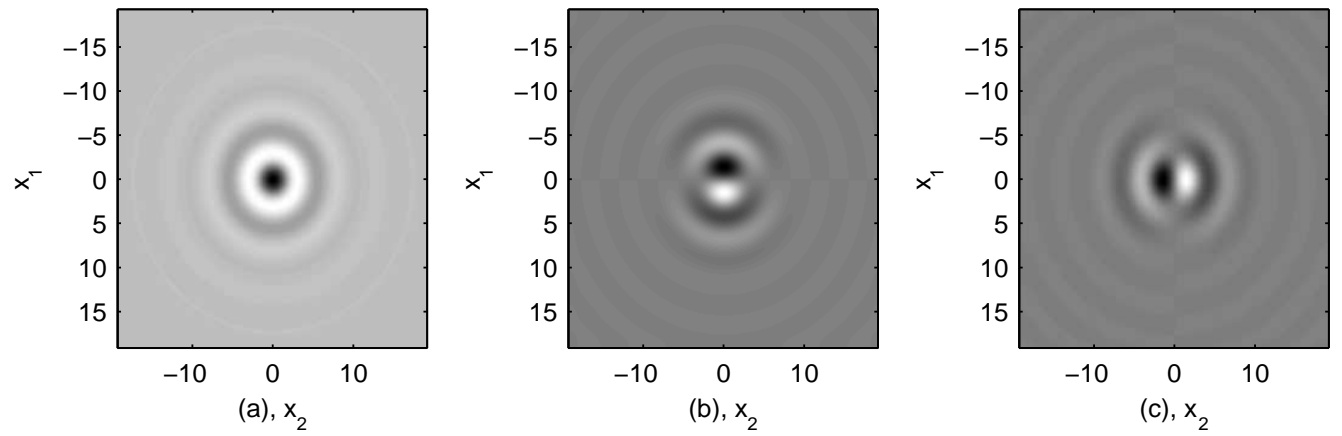

Fig. 3. The real part (a), and two imaginary parts, (b) and (c), of the isotropic monogenic Morse wavelets, with $n=0, l=9$ and $m=4$.

For a plot of the hypercomplexing isotropic Morse wavelets see Figure 2 It is clear that these wavelets have an isotropic support but recognize variation associated with the $x_{1}, x_{2}$ and diagonal directions.

Definition 3.6: Isotropic monogenic Morse wavelets.

The $l$, and $m$, isotropic monogenic Morse wavelets are defined in the frequency domain for $n \in \mathbb{N}$ by

$$
\Psi_{I, n ; l, m}^{(+)}(\mathbf{f})=(1-\boldsymbol{k} \cos (\phi)+\sin (\phi)) \Psi_{n ; l, m}^{(e)}(f) .
$$

By using the inverse Hankel transform the real part of the wavelets may be retrieved in the spatial domain:

$$
\psi_{I, n ; l, m}^{(e)}(\mathbf{x})=A_{n ; l, m}^{\prime} 2 \pi\left[\int_{0}^{\infty} d f f(2 \pi f)^{l} e^{-(2 \pi f)^{m}} \mathrm{~L}_{k}^{c}\left[2(2 \pi f)^{m}\right] J_{0}(2 \pi f x)\right] .
$$

The monogenic extension of the $n$-th order mother Morse wavelet is found by

$$
\psi_{I, n ; l, m}^{(+)}(\mathbf{x})=\psi_{n ; l, m}^{(e)}(\mathbf{x})+(\boldsymbol{i} \cos \chi+\boldsymbol{j} \sin \chi) 2 \pi\left[\int_{0}^{\infty} d f f \Psi_{n ; l, m}^{(e)}(\mathbf{f}) J_{1}(2 \pi f x)\right] .
$$

Exact forms for these functions for special choices of parameters can be determined analytically (see Appendix II-D). Figure 3 displays a plot of the isotropic monogenic functions: it is clear that the wavelets have an isotropic support but recognize variation associated with the two spatial directions. Note that the Poisson scale space introduced by Felsberg and Sommer [47] is not a special case of a isotropic monogenic Morse wavelet as their analysis function is not zero at $\mathbf{f}=\mathbf{0}$, and would require setting $l=0$.

Definition 3.7: Directional monogenic Morse wavelets.

The $\beta$, and $\gamma$, directional monogenic Morse wavelets are for $n \in \mathbb{N}$ defined by

$$
\begin{aligned}
\psi_{D, n ; \beta, \gamma}^{(+)}(\mathbf{x})= & R_{\pi / 4}\left\{\psi_{1 D, n ; \beta, \gamma}^{(e)}\left(x_{1}\right) \psi_{1 D, n ; \beta, \gamma}^{(e)}\left(x_{2}\right)-\psi_{1 D, n ; \beta, \gamma}^{(o)}\left(x_{1}\right) \psi_{1 D, n ; \beta, \gamma}^{(o)}\left(x_{2}\right)\right\} \\
& +\boldsymbol{i} \mathcal{R}_{1}\left\{R_{\pi / 4}\left\{\psi_{1 D, n ; \beta, \gamma}^{(e)}\left(x_{1}\right) \psi_{1 D, n ; \beta, \gamma}^{(e)}\left(x_{2}\right)-\psi_{1 D, n ; \beta, \gamma}^{(o)}\left(x_{1}\right) \psi_{1 D, n ; \beta, \gamma}^{(o)}\left(x_{2}\right)\right\}\right\} .
\end{aligned}
$$

As expected $\psi_{D, 0 ; 9,3}^{(+)}(\mathbf{x})$ corresponds to a complex directional wavelet, and is given by Figures 4 (a) and (b), where the other quaternionic components are identically zero.

Definition 3.8: Hypercomplex Morse wavelets.

The $\beta$, and $\gamma$, hypercomplex Morse wavelets are for any integer valued $n$ defined by

$$
\begin{aligned}
\psi_{D, n ; \beta, \gamma}^{(++)}(\mathbf{x})= & R_{\pi / 4}\left\{\psi_{1 D, n ; \beta, \gamma}^{(e)}\left(x_{1}\right) \psi_{1 D, n ; \beta, \gamma}^{(e)}\left(x_{2}\right)-\psi_{1 D, n ; \beta, \gamma}^{(o)}\left(x_{1}\right) \psi_{1 D, n ; \beta, \gamma}^{(o)}\left(x_{2}\right)\right\} \\
& +\boldsymbol{i}\left[R_{\pi / 4}\left\{\psi_{1 D, n ; \beta, \gamma}^{(o)}\left(x_{1}\right) \psi_{1 D, n ; \beta, \gamma}^{(e)}\left(x_{2}\right)+\psi_{1 D, n ; \beta, \gamma}^{(e)}\left(x_{1}\right) \psi_{1 D, n ; \beta, \gamma}^{(o)}\left(x_{2}\right)\right\}\right] .
\end{aligned}
$$

$\psi_{D, 0 ; 9,3}^{(++)}(\mathbf{x})$, is thus calculated from $\psi_{1 D, 0 ; 9,3}^{(e)}\left(x_{1}\right)$ and $\psi_{1 D, 0 ; 9,3}^{(o)}\left(x_{1}\right)$ in equation 69 , and plotted in terms of its real and imaginary component see Figures 4 (a) and (c). The real component is similar to $\psi_{D, 0 ; 9,3}^{(+)}(\mathbf{x})$, but recall that the two imaginary components are not equal, i.e. $\psi_{D, 2,0 ; 9,3}(\mathbf{x}) \neq \psi_{D, 3,0 ; 9,3}(\mathbf{x})$. Directional wavelets have been previously constructed from wavelets related to the 1-D Morse wavelets, see work by Antoine et al: [20], [21], [68], but these are distinct from $\psi_{D, n ; \beta, \gamma}^{(+)}(\mathbf{x})$, as well as $\psi_{D, n ; \beta, \gamma}^{(++)}(\mathbf{x})$. The wavelets defined by Antoine et al. [20][p. 319] are based on the generalized 1-D Morse wavelets with $\beta=l=m$ and $\gamma=1$, and are referred to as 2-D Cauchy wavelets. In their construction a covariant change of axes has been implemented to make the wavelets compactly supported on a cone in the frequency domain, rather than by rotating a function supported in the $45^{\circ}$, or $\pi / 4$ line. 


\section{Choice of mother wavelet function}

The Morse wavelets may be considered as optimally localised over a given region of space and spatial frequency space, see for example Olhede \& Walden [33] and Metikas \& Olhede [44]. Note that the hyperanalyticizing 2-D extensions of the real mother wavelet functions are localised to the same region as the real wavelet functions. In order to choose an analysis mother wavelet, we either take a real mother wavelet that is isotropic or one which is anisotropic. If the directional choice of localisation is necessary to disentangle local structure, we use a combinations of the tensor products of 1-D wavelets. However, if the isotropic choice of localisation is sufficient to separate components at given scale and spatial positions, then we base the quaternionic filters on the isotropic Morse wavelet.

For a pre-specified spatial and spatial frequency region of either a directional or isotropic form we choose $\beta$ and $\gamma$, or equivalently $l$ and $m$, to calculate a suitable family of even or isotropic wavelets that are optimally local to a given region (for a more thorough discussion see [33] or [44]). Thus $\beta, \gamma$, or $l$ and $m$, should be chosen depending on how the image should be localised in space and spatial frequency. As a second stage a suitable quaternionic extension of the real-valued mother wavelet is chosen. The choice of quaternionic extension will depend on what assumptions can be made regarding the underlying structure of the observed local variation. As may be recalled from Figures 1, 2, 3 and 4, each quaternionic extension represents very different local oscillatory structures associated with the same period. The separable hypercomplexing wavelets are supported in two orthogonal directions, each real component of the quaternionic wavelet capable of extracting even/odd and diagonal structure. The separable filters resemble the quaternionic Gabor decomposition suggested by von Bülow and Sommer [49], and the wavelet coefficients represent structure separable in a rotated coordinate frame. The directional hyperanalyticizing wavelets are constructed from a tensor product of 1-D wavelets, but where the directional wavelets are a sum of 1-D even and odd function tensor products, as was suggested by Selesnick et al [1]. Local plane waves, isolated in a given direction can be represented well by such a decomposition. The isotropic wavelets mix energy isotropically, i.e. localise an image isotropically. Both the hypercomplex and monogenic wavelets are built from the idea that the real wavelet isolates a single component by its scale and spatial localisation - subsequently the isolated components will be represented by their plane-wave (monogenic), or separable wave (hypercomplex) polar representation. This method resembles a Hilbert spectrum representation of a function in 1-D [34]. Once the wavelet transform coefficients have been calculated at a single orientation, if the assumption of single isolated structure holds, then no further calculations are necessary. This represents a substantial reduction in computational time. Once the CWT coefficients have been calculated, the local structure of the analysed image is represented in terms of the quaternionic transform coefficients, and their polar representation.

\section{QUATERNIONIC WAVELET COEFFICIENTS}

Having constructed various quaternionic mother wavelets, each suitable for the analysis of local structure, we outline the properties enjoyed by the coefficients. Equation (3) defined the CWT coefficients of image $g(\mathbf{x})$ analysed with a wavelet family constructed from wavelet $\psi(\mathbf{x})$. If $\psi(\mathbf{x})$ is quaternionic then equation (3) takes the form:

$$
\begin{aligned}
w_{\psi}(\boldsymbol{\xi} ; g) & =\int_{-\infty}^{\infty} \int_{-\infty}^{\infty} g(\mathbf{x}) \psi_{\boldsymbol{\xi}}^{*}(\mathbf{x}) d^{2} \mathbf{x} \\
& =\int_{-\infty}^{\infty} \int_{-\infty}^{\infty} g(\mathbf{x})\left[\psi_{\boldsymbol{\xi}}^{(r)}(\mathbf{x})-\boldsymbol{i} \psi_{\boldsymbol{\xi}}^{(i)}(\mathbf{x})-\boldsymbol{j} \psi_{\boldsymbol{\xi}}^{(j)}(\mathbf{x})-\boldsymbol{k} \psi_{\boldsymbol{\xi}}^{(k)}(\mathbf{x})\right] d^{2} \mathbf{x} \\
& =w_{\psi}^{(r)}(\boldsymbol{\xi} ; g)-\boldsymbol{i} w_{\psi}^{(i)}(\boldsymbol{\xi} ; g)-\boldsymbol{j} w_{\psi}^{(j)}(\boldsymbol{\xi} ; g)-\boldsymbol{k} w_{\psi}^{(k)}(\boldsymbol{\xi} ; g),
\end{aligned}
$$

thus defining $w_{\psi}^{(u)}(\boldsymbol{\xi} ; g), \quad u=r, i, j, k$. The local structure at $\boldsymbol{\xi}$ is given a four-vector valued representation, via some phase/s representations or relative magnitudes of the $w_{\psi}^{(u)}(\boldsymbol{\xi} ; g)$. 'Local energy' is determined from the local magnitude, $\left|w_{\psi}(\boldsymbol{\xi} ; g)\right|$, of the image $g(\mathbf{x})$. Define $\boldsymbol{\zeta}=\left[a, \theta, \mathbf{f}_{b}\right]^{T}$, where $\mathbf{f}_{b}$ is the Fourier variable after the Fourier transform in $\boldsymbol{b}$ has been implemented, and $\boldsymbol{\kappa}=\left[a, \theta, \boldsymbol{q}_{b}\right]^{T}$, where $\boldsymbol{q}_{b}$ is the quaternion Fourier variable of $\boldsymbol{b}$.

Definition 4.1: Scalogram of $g(\mathbf{x})$.

The local energy of $g(\mathbf{x})$ at $\boldsymbol{\xi}$ is defined by the scalogram and is given by

$$
S_{\psi}(\boldsymbol{\xi} ; g)=\left|w_{\psi}(\boldsymbol{\xi} ; g)\right|^{2}=w_{\psi}^{(r) 2}(\boldsymbol{\xi} ; g)+w_{\psi}^{(i) 2}(\boldsymbol{\xi} ; g)+w_{\psi}^{(j) 2}(\boldsymbol{\xi} ; g)+w_{\psi}^{(k) 2}(\boldsymbol{\xi} ; g) \text {. }
$$



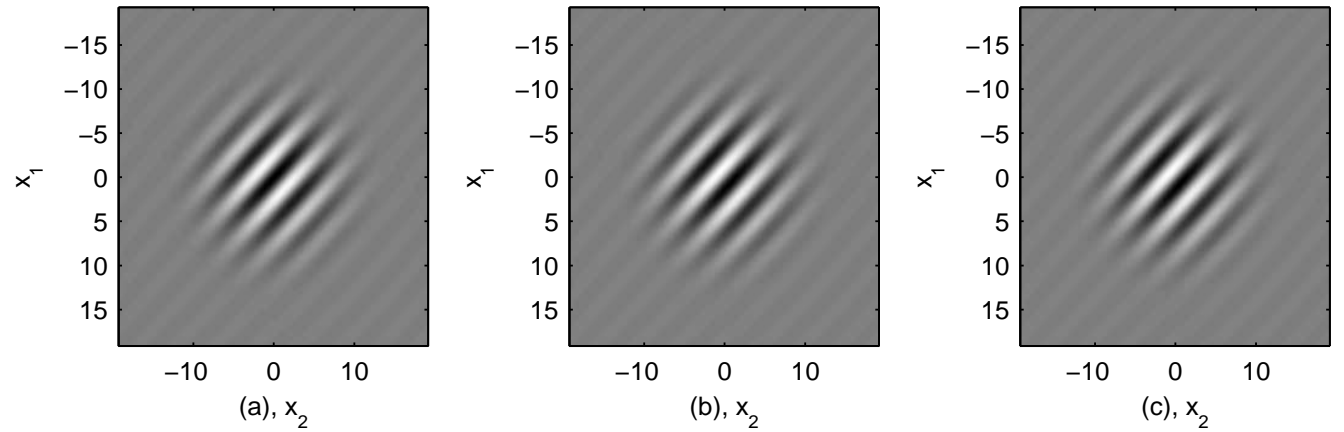

Fig. 4. The real part $\psi_{D, 0 ; 9,4}(\mathbf{x})((\mathrm{a}))$, and first imaginary parts $\left((\mathrm{b}) \psi_{D, 3,0 ; 9,4}(\mathbf{x})\right.$ and (c) $\left.\psi_{D, 2,0 ; 9,4}(\mathbf{x})\right)$ of the directional monogenic Morse wavelet and the hypercomplex Morse wavelet, with $n=0, \beta=9$ and $\gamma=4$.

Irrespective of what quaternionic mother wavelet function is used, the magnitude square of the coefficients is interpreted as local signal presence. The interpretation is appropriate as the four wavelet functions $\psi^{(u)}(\mathbf{x})$ with $u=r, i, j, k$, are chosen to be local to $\mathbf{x}=\mathbf{b}$ and scale $a$, where the localisation of $\psi^{(u)}(\mathbf{x})$ with $u=i, j, k$, is similar to that of $\psi^{(r)}(\mathbf{x})$ from our previous comments in sections $\llbracket-\mathrm{H}$ and $\amalg-\mathrm{F}$ Note that $-\psi^{(o o)}(\mathbf{x})$ enjoys the same localization as $\psi^{(o o)}(\mathbf{x})$.

The relative relationships between the other components of the quaternionic wavelet will depend on the choice of mother wavelet function used to decompose the image, and so the phase representation of structure will vary with the mother wavelets used. To obtain coefficients with interpretable phase relations we calculate $w_{\psi}(\boldsymbol{\xi} ; g)$, so that it corresponds to a $\theta$-hyperanalytic signal in index $\mathbf{b}$ for any fixed value of $\theta$ and $a$. The signal $g(\cdot)$ could also be represented in terms of some weights attached with functions $\psi_{\boldsymbol{\xi}}(\cdot)$, that are hyperanalytic in $\mathbf{x}$ for any fixed $\xi_{0}$. In the latter case equation (3) states that the signal may be reconstructed in terms of the weighted and rotated hyperanalytic functions $\psi_{\boldsymbol{\xi}}(\cdot)$. In 1-D these two perspectives coincide, as analysis of a signal in terms of a analytic wavelet yields both analytic wavelet coefficients and a reconstruction in terms of the analytic wavelet functions. In 2-D the problem is more complicated, due to the non-zero $\boldsymbol{k}$ component in the hypercomplexing wavelet. We introduce the additional notation of $\boldsymbol{J}_{\pi / 2} \boldsymbol{\kappa} \equiv\left[a, \theta, \boldsymbol{J}_{\pi / 2} \mathbf{q}_{b}\right]^{T}$.

Proposition 12: Forms of the FT and QFT of wavelet coefficients with real wavelet. The FT and QFT of $w_{\psi}^{(r)}(\boldsymbol{\xi} ; g)$ for a real signal $g(\mathbf{x})$ with a real-valued wavelet $\psi^{(r)}(\cdot)$ respectively are

$$
\begin{aligned}
W_{\psi}^{(r)}(\boldsymbol{\zeta} ; g) & =G\left(\mathbf{f}_{b}\right) a \Psi^{(r) *}\left(a \boldsymbol{r}_{-\theta} \mathbf{f}_{b}\right), \\
W_{\psi ; Q}^{(r)}(\boldsymbol{\kappa} ; g) & =\frac{1-\boldsymbol{k}}{2} G\left(\mathbf{q}_{b}\right) a \Psi^{(r) *}\left(a \boldsymbol{r}_{-\theta} \mathbf{q}_{b}\right)+\frac{1+\boldsymbol{k}}{2} G\left(\boldsymbol{J}_{\pi / 2} \mathbf{q}_{b}\right) a \Psi^{(r) *}\left(a \boldsymbol{r}_{-\theta} \boldsymbol{J}_{\pi / 2} \mathbf{q}_{b}\right) \\
& =\frac{1-\boldsymbol{k}}{2} W_{\psi}^{(r)}(\boldsymbol{\kappa} ; g)+\frac{1+\boldsymbol{k}}{2} W_{\psi}^{(r)}\left(\boldsymbol{J}_{\pi / 2} \boldsymbol{\kappa} ; g\right) .
\end{aligned}
$$

Proof: Equations (90) and 910 follow by direct calculation, and are only stated so that a comparison can be made with the quaternionic wavelet coefficients.

Proposition 13: Forms of the FT and QFT of wavelet coefficients with quaternionic wavelet. The FT and QFT of $w_{\psi}(\boldsymbol{\xi} ; g)$ for a real signal $g(\mathbf{x})$ with a quaternion-valued wavelet $\psi(\cdot)$ respectively are,

$$
\begin{aligned}
W_{\psi}(\boldsymbol{\zeta} ; g)= & G\left(\mathbf{f}_{b}\right)\left[a \Psi^{(r) *}\left(a \boldsymbol{r}_{-\theta} \mathbf{f}_{b}\right)-\boldsymbol{i} a \Psi^{(i) *}\left(a \boldsymbol{r}_{-\theta} \mathbf{f}_{b}\right)-j a \Psi^{(j) *}\left(a \boldsymbol{r}_{-\theta} \mathbf{f}_{b}\right)-\boldsymbol{k} a \Psi^{(k) *}\left(a \boldsymbol{r}_{-\theta} \mathbf{f}_{b}\right)\right] \\
W_{\psi ; Q}(\boldsymbol{\kappa} ; g)= & \frac{1}{2} W_{\psi}(\boldsymbol{\kappa} ; g)-\frac{\boldsymbol{k}}{2} W_{\psi^{(r)}-i \psi^{(i)}+j \psi^{(j)}-k \psi^{(k)}}(\boldsymbol{\kappa} ; g)+\frac{1}{2} W_{\psi}\left(\boldsymbol{J}_{\pi / 2} \boldsymbol{\kappa} ; g\right) \\
& +\frac{\boldsymbol{k}}{2} W_{\psi^{(r)}-i \psi^{(i)}+j \psi^{(j)}-k \psi^{(k)}}\left(\boldsymbol{J}_{\pi / 2} \boldsymbol{\kappa} ; g\right) \\
\neq & \frac{1-\boldsymbol{k}}{2} W_{\psi}(\boldsymbol{\kappa} ; g)+\frac{1+\boldsymbol{k}}{2} W_{\psi}\left(\boldsymbol{J}_{\pi / 2} \boldsymbol{\kappa} ; g\right) .
\end{aligned}
$$

Proof: See appendix 【II-A Equations 92 and (93) illustrate the new properties afforded by the quaternionic decomposition, in comparison to a real-valued decomposition of $g(\boldsymbol{x})$. The $\boldsymbol{i}$ and $\boldsymbol{k}$ coefficients will enable us to characterise structure in the $x_{1}$ axis, and we are not constrained to average the coefficients over $x_{1}$ and $-x_{1}$, 
$c f$ equation (91). With a well-chosen quaternionic mother wavelet this will give us tools to represent oscillatory structures.

\section{A. Stability to changes of axes}

The axes of observation are not necessarily aligned with the local axes of variation in the image. The CWT is covariant with respect to the transformation $\mathbf{x} \mapsto \boldsymbol{r}_{-\theta^{\prime}} \mathbf{x}-\mathbf{x}^{\prime}$, i.e. if we observe $g(\mathbf{x})=g_{2}\left(\boldsymbol{r}_{-\theta^{\prime}}\left(\mathbf{x}-\mathbf{x}^{\prime}\right)\right)$, rather than $g_{2}(\mathbf{x})$, then using equation (2.18) of [21][p. 262] on each of the real valued weights associated with $1, \boldsymbol{i}, \boldsymbol{j}$, and $\boldsymbol{k}$, we may formally note that:

$$
w_{\psi}\left(\boldsymbol{\xi} ; g(\mathbf{x})=w_{\psi}\left(a, \theta-\theta^{\prime}, \boldsymbol{r}_{-\theta^{\prime}} \mathbf{b}-\mathbf{x}^{\prime} ; g_{2}(\mathbf{x})\right) .\right.
$$

Having noted this equivariance of the quaternionic CWT, one might assume that no discussion needs to be provided of changes of axes of observation. However, a discretization of the calculation of the CWT, implies that we calculate the CWT coefficients at a sampled subset of all values of the locality index $\boldsymbol{\xi}$. Small misalignments in space between the wavelet function and the object under observation may cause the CWT coefficient at a given value of $\boldsymbol{\xi}$ to be small even if the signal has a large contribution at $\mathbf{x}=\mathbf{b}$ and $\mathbf{f}=a^{-1} \boldsymbol{r}_{-\theta} \mathbf{f}_{0}$. The down-sampling inherent in most filter bank implementations of the CWT exacerbates the spatial initialization problem. In 1-D time shift variance has been considered in great detail see for example [22], [24], [75], and small misalignment in time may be considered in terms of shifts in phase. In particular, [22][p. 1794] discusses the relationship between small spatial shifts and phase shifts. It is important that, at any given $\boldsymbol{\xi}$, the transform of structure that corresponds to the same space and spatial frequency locality should not correspond to very different $\left|w_{\psi}(\boldsymbol{\xi} ; g(\mathbf{x}))\right|$ due to small misalignments in space between the image and the wavelet. This becomes equivalent to requiring that the magnitude of the CWT does not change under phase shifts of the signal.

In 1-D it was shown in equation (6.11) and the subsequent discussion in [56] that, if a real signal of sinusoidal form is phase shifted by a constant angle $\theta_{s}$, the analytic CWT of this signal will correspond to a complex phase times the CWT of the original signal. This ensures CWT magnitude invariance to phase shifts, and produces a stable CWT. We shall see that the hyperanalytic wavelet coefficients exhibit similar structure, but the definition of a phase-shifted signal in 2-D will vary with the choice of local structure. Depending on the local structure of the image a phase shift will be defined as a single shift in a given direction, or as two shifts in perpendicular directions.

\section{B. The hypercomplex $C W T$}

The hypercomplex signal of a real image $g(\mathbf{x})$ represents a real-valued image in terms of a four-vector representing local structure, where the local variation in the image is considered in terms of the $x_{1}$ and $x_{2}$ axes separately, as well as joint variation in the two axes. When the $\theta$-hypercomplex signal is formed, variations in a rotated frame of reference are considered, and this naturally introduces $\widetilde{g}_{-\theta}(\mathbf{x})$, rather than $g(\mathbf{x})$ as the object under analysis. If there are separable oscillations present in $g(\mathbf{x})$ at a spatial position $\mathbf{x}$, in direction along and perpendicular to the axes rotated by $\theta$, then using the polar representation of the $\theta$-hypercomplex signal given in equation (28) will allow for the characterisation of the local structure. The local structure is, in this instance, given in terms of the amplitude or energy associated with the variation, the period in the first axis' oscillation, given by $\widetilde{\alpha}_{-\theta, \theta}(\mathbf{x})$, as well as the period in the second axis' oscillation, given by $\widetilde{\beta}_{-\theta, \theta}(\mathbf{x})$. If there truly is a completely separable structure present, then $\widetilde{\gamma}_{-\theta, \theta}(\mathbf{x})$ will confirm this assumption by taking the value of zero. If not, a non-zero value of $\widetilde{\gamma}_{-\theta, \theta}(\mathbf{x})$ will characterise the non-separability of the oscillation. By recalculating the decomposition at different values of $\theta \in[0, \pi / 2]$, the orientation may be determined from the analysed image, as the value of $\theta$ that yields $\widetilde{\gamma}_{-\theta, \theta}(\mathbf{x})=0$. Unfortunately most observed images do not correspond to the simple structure of separable variation at any given $\mathbf{x}$, but variation exists at many different spatial scales and orientations at the same spatial position. For such images some localisation needs to be implemented when forming the hypercomplex representation of the image variation. For an image corresponding to variation at many different scales, the full structure of the image at a given spatial position will not be well represented in terms of an amplitude, and two periods. Once different structures associated with given variations have been isolated in the image, the structure of each component may be given a hypercomplex representation. Under the assumption of local separability the hypercomplex CWT, or HCWT, will be calculated. 
To isolate the individual component we chose to combine the hypercomplex representation of an image with the CWT. The wavelet will localise the image in spatial position, scale and potentially orientation, and is assumed to be sufficient to separate the different components. In theory, to construct the HCWT, the CWT may be combined with the hypercomplex representation in many different orders. We could start by calculating the hypercomplex signal annihilating all anti-hypercomplex components in equation (27), and then localise the signal in position, scale and orientation with a real valued wavelet, with the aim that once localised the CWT would isolate a single component that would naturally be given in polar form. We could equivalently argue that we should first project the image in position, scale and orientation using a real wavelet to isolate a single component and then find the hypercomplex representation of the localised in $\mathbf{b}$ signal. It is most convenient to use a single wavelet decomposition that produces quaternionic coefficients in one calculation. Fortunately, a single quaternionic wavelet which is not itself hypercomplex can be chosen so that both operations can be done in a single step. The three procedures give the same filtered image, and this defines a local $\theta$-hypercomplex signal with interpretable magnitude and phase structure. To demonstrate the properties of the coefficients it is most easy to work in the Fourier and quaternionic Fourier domains.

Proposition 14: FT of HCWT.

The CWT of the real signal $g(\mathbf{x})$ using the hypercomplexing wavelet $\psi^{++}(\cdot)$ has a Fourier transform given by

$$
W_{\psi}^{(++)}(\boldsymbol{\zeta} ; g)=\left[1+\operatorname{sgn}\left(\left[\boldsymbol{r}_{-\theta} \mathbf{f}_{b}\right]_{2}\right)\right]\left[1-\boldsymbol{k} \operatorname{sgn}\left(\left[\boldsymbol{r}_{-\theta} \mathbf{f}_{b}\right]_{1}\right)\right] W_{\psi}^{(e e)}(\boldsymbol{\zeta} ; g)
$$

Proof: The result follows by direct calculation. The hypercomplexing wavelet is filtering the signal in the rotated coordinate system, constructing a signal locally odd in $\left(\boldsymbol{r}_{-\theta} \mathbf{x}\right)_{1}$ as the $\boldsymbol{i}$ component, a signal locally odd in the $\left(\boldsymbol{r}_{-\theta} \mathbf{x}\right)_{2}$ as the $\boldsymbol{j}$ component and a signal locally odd in $\left(\boldsymbol{r}_{-\theta} \mathbf{x}\right)_{1}$ and $\left(\boldsymbol{r}_{-\theta} \mathbf{x}\right)_{2}$ as the $\boldsymbol{k}$ component.

Proposition 15: QFT of HCWT .

The CWT of the real signal $g(\mathbf{x})$ using the hypercomplexing wavelet $\psi^{++}(\cdot)$ has QFT given by

$$
\begin{aligned}
W_{Q, \psi}^{(++)}(\boldsymbol{\kappa} ; g)= & \frac{1-\boldsymbol{k}}{2}\left(1+\operatorname{sgn}\left(\left[\boldsymbol{r}_{-\theta} \mathbf{q}_{b}\right]_{1}\right)\right)\left(1+\operatorname{sgn}\left(\left[\boldsymbol{r}_{-\theta} \mathbf{q}_{b}\right]_{2}\right)\right) W_{\psi}^{(e e)}(\boldsymbol{\kappa} ; g) \\
& +\frac{1+\boldsymbol{k}}{2}\left(1+\operatorname{sgn}\left(\left[\boldsymbol{r}_{\theta} \mathbf{q}_{b}\right]_{1}\right)\right)\left(1+\operatorname{sgn}\left(\left[\boldsymbol{r}_{\theta} \mathbf{q}_{b}\right]_{2}\right)\right) W_{\psi}^{(e e)}\left(\boldsymbol{J}_{\pi / 2} \boldsymbol{\kappa} ; g\right) .
\end{aligned}
$$

Proof: The proof of this calculation follows by direct calculation. Comparing this equation with equation (25) we observe that this is a $\theta$-hypercomplex function, i.e. hypercomplex in a rotated frame of reference in terms of

$$
H\left(\boldsymbol{r}_{-\theta} \mathbf{q}\right)=a \Psi^{(e e) *}\left(a \boldsymbol{r}_{-\theta} \mathbf{q}\right) G(\mathbf{q}), H\left(\mathbf{q}_{1}\right)=a \Psi^{(e e)}\left(a \mathbf{q}_{1}\right) G\left(\boldsymbol{r}_{\theta} \mathbf{q}_{1}\right) \equiv a \Psi^{(e e)}\left(a \mathbf{q}_{1}\right) \widetilde{G}_{-\theta}\left(\mathbf{q}_{1}\right) .
$$

The hypercomplex CWT simultaneously constructs a $\theta$-hypercomplex signal as localising the signal in frequency. $h(\mathbf{b})$ corresponds to the CWT of $\widetilde{g}_{-\theta}(\mathbf{x})$, calculated at rotation $\theta=0$, and we may also interpret $w_{\psi}^{(++)}(\boldsymbol{\xi} ; g)$ as the $\theta$-hypercomplex extension of the real CWT with $\theta=0$ of the signal $\widetilde{g}_{-\theta}(\mathbf{x})$, i.e. calculating the real CWT of the real signal at $\boldsymbol{\kappa}_{0}=[a, 0, \mathbf{b}]^{T}$, and then extending it to a $\theta$-hypercomplex signal. By equation (25) we note:

$$
\begin{aligned}
\left(W_{Q, \psi}^{(e e)}\left(\boldsymbol{\kappa}_{0} ; \widetilde{g}_{-\theta}\right)\right)_{\theta}^{(++)}= & \left(1+\operatorname{sgn}\left(\left[\boldsymbol{r}_{-\theta} \mathbf{q}_{b}\right]_{1}\right)\right)\left(1+\operatorname{sgn}\left(\left[\boldsymbol{r}_{-\theta} \mathbf{q}_{b}\right]_{2}\right)\right)\left(\frac{1-\boldsymbol{k}}{2}\right) W_{\psi}^{(e e)}(\boldsymbol{\kappa} ; g) \\
& +\left(1+\operatorname{sgn}\left(\left[\boldsymbol{r}_{\theta} \mathbf{q}_{b}\right]_{1}\right)\right)\left(1+\operatorname{sgn}\left(\left[\boldsymbol{r}_{\theta} \mathbf{q}_{b}\right]_{2}\right)\right)\left(\frac{1+\boldsymbol{k}}{2}\right) W_{\psi}^{(e e)}\left(\boldsymbol{J}_{\pi / 2} \boldsymbol{\kappa} ; g\right) .
\end{aligned}
$$

Thus $w_{\psi}^{(++)}(\boldsymbol{\xi} ; g)$ is a $\theta$-hypercomplex signal and corresponds to constructing the $\theta$-hypercomplex extension of $w_{\psi}^{(e e)}\left(\boldsymbol{\xi}_{0} ; \widetilde{g}_{-\theta}(\mathbf{x})\right)$. This gives the interpretation of the coefficients as the appropriate hyperanalytic extension of a signal local to $\boldsymbol{\xi}_{0}=[a, 0, \boldsymbol{\beta}]$ existing, and naturally described, in direction $\theta$.

Proposition 16: The FT of the HCWT of the $\theta$-hypercomplex signal.

The Fourier transform of the hypercomplex CWT of the $\theta$ hypercomplex signal takes the form:

$$
W_{\psi}^{(++)}\left(\boldsymbol{\zeta} ; \widetilde{g}_{-\theta, \theta}^{(++)}\right)=2\left[1+\operatorname{sgn}\left(\left[\boldsymbol{r}_{-\theta} \mathbf{f}_{b}\right]_{2}\right)\right]\left[1-\boldsymbol{k} \operatorname{sgn}\left(\left[\boldsymbol{r}_{-\theta} \mathbf{f}_{b}\right]_{1}\right)\right] W_{\psi}^{(e e)}(\boldsymbol{\zeta}, g) .
$$

Proof: See appendix III-B This results is intermediary to proving that the order of operations of constructing the hypercomplex signal and calculating the CWT is not important. The appendix gives the form of $W_{\psi}^{(++)}\left(\boldsymbol{\zeta} ; \widetilde{g}_{-\theta, \theta}^{\left(\mu_{1} \mu_{2}\right)}\right)$, for $\mu_{1}$ and $\mu_{2}$ taking any of the values \pm . For the 1-D signal, only the analytic wavelet transform of the analytic 
signal was non-null - the analytic wavelet annihilated the anti-analytic component. This is not the case for a hypercomplex signal, as in not all three of the anti-hypercomplex components are annihilated.

Proposition 17: The FT of the real even CWT of the $\theta$-hypercomplex signal.

The Fourier transform of the hypercomplex CWT of the $\theta$-hypercomplex signal takes the form:

$$
W_{\psi}^{(e e)}\left(\boldsymbol{\zeta} ; \widetilde{g}_{-\theta, \theta}^{(++)}\right)=\left(1+\operatorname{sgn}\left(\left[\boldsymbol{r}_{-\theta} \mathbf{f}_{b}\right]_{2}\right)\right)\left(1-\boldsymbol{k} \operatorname{sgn}\left(\left[\boldsymbol{r}_{-\theta} \mathbf{f}_{b}\right]_{1}\right)\right) W_{\psi}^{(e e)}(\boldsymbol{\zeta} ; g) .
$$

Proof: See section [II-C We interpret $w_{\psi}^{(e e)}\left(\boldsymbol{\xi} ; \widetilde{g}_{-\theta, \theta}^{(++)}\right)$as the localised $\theta$-hypercomplex signal of the signal observed in a different axes: this should have a polar representation that is representative of the signal structure.

Theorem 4: Construction of the local $\theta$-hypercomplex signal.

By calculating the hypercomplex CWT of a real signal $g(\mathbf{x})$, a local $\theta$-hypercomplex signal is constructed.

Proof: See appendix

$$
\begin{aligned}
W_{\psi}^{(++)}(\boldsymbol{\zeta} ; g) & =\left[W_{\psi}^{(e e)}\left(\boldsymbol{\zeta}_{0} ; \widetilde{g}_{-\theta}\right)\right]_{\theta}^{(++)}=\frac{1}{2} W_{\psi}^{(++)}\left(\boldsymbol{\zeta} ; \widetilde{g}_{-\theta, \theta}^{(++)}\right)=W_{\psi}^{(e e)}\left(\boldsymbol{\zeta} ; \widetilde{g}_{-\theta, \theta}^{(++)}\right), \\
W_{\psi, Q}^{(++)}(\boldsymbol{\kappa} ; g) & =\left[W_{\psi, Q}^{(e e)}\left(\boldsymbol{\kappa}_{0} ; \widetilde{g}_{-\theta}\right)\right]_{\theta}^{(++)}=\frac{1}{2} W_{\psi, Q}^{(++)}\left(\boldsymbol{\kappa} ; \widetilde{g}_{-\theta, \theta}^{(++)}\right)=W_{\psi, Q}^{(e e)}\left(\boldsymbol{\kappa} ; \widetilde{g}_{-\theta, \theta}^{(++)}\right), \\
w_{\psi}^{(++)}(\boldsymbol{\xi} ; g) & =\left[w_{\psi}^{(e e)}\left(\boldsymbol{\xi}_{0} ; \widetilde{g}_{-\theta}\right)\right]_{\theta}^{(++)}=\frac{1}{2} w_{\psi}^{(++)}\left(\boldsymbol{\xi} ; \widetilde{g}_{-\theta, \theta}^{(++)}\right)=w_{\psi}^{(e e)}\left(\boldsymbol{\xi} ; \widetilde{g}_{-\theta, \theta}^{(++)}\right) .
\end{aligned}
$$

Once one the above three sets of equations in (100) is proved, the rest follow through Fourier, Inverse Fourier, Quaternionic Fourier, and Inverse Quaternionic Fourier transforms. Furthermore the equations shed light on the interpretation of $w_{\psi}^{(++)}(\boldsymbol{\xi} ; g)$.

$\left[w_{\psi}^{(e e)}\left(\boldsymbol{\xi}_{0} ; \widetilde{g}_{-\theta}\right)\right]_{\theta}^{(++)}$is a $\theta$-hypercomplex signal by proposition 15 , thus its polar representation is meaningful, as it corresponds to a real signal that has been localised in scale, and extended to a $\theta$-hypercomplex signal. Furthermore $w_{\psi}^{(e e)}\left(\boldsymbol{\xi} ; \widetilde{g}_{-\theta, \theta}^{(++)}\right)$can be viewed as the local contribution of the $\theta$-hypercomplex signal, and is therefore also meaningful if there is signal presence at that point. These two quantities are both equal to half $w_{\psi}^{(++)}\left(\boldsymbol{\xi} ; \widetilde{g}_{-\theta, \theta}^{(++)}\right)$, the hypercomplex CWT of the $\theta$-hypercomplex signal. Thus $w_{\psi}^{(++)}(\boldsymbol{\xi} ; g)$ has an interpretation in terms of $\widetilde{g}_{-\theta, \theta}(\mathbf{x})$, the signal naturally represented in the rotated coordinate system, but observed in another coordinate system. $w_{\psi}^{(++)}(\boldsymbol{\xi} ; g)$ can thus be used to locally at $\boldsymbol{\xi}$ represent a real-valued signal in terms of phase and amplitude. Unlike von Bülow and Sommer, we need not assume that a single component is present at $\mathbf{x}=\boldsymbol{b}$, and it suffices to assume that once $g(\mathbf{x})$ has been localised to $(a, \theta)$ and $\mathbf{x}=\mathbf{b}$, then a single component is present. Then $w_{\psi}^{(++)}(\boldsymbol{\xi} ; g)$ can be represented in polar form using equation (28). Furthermore, if we assume that the signal corresponds to a locally separable oscillation, the following theorem specifies its representation.

Theorem 5: The HCWT of a separable oscillatory signal.

The hypercomplex CWT of an oscillatory signal modelled with $\mathbf{x}^{\prime}=\boldsymbol{r}_{-\theta} \mathbf{x}$ as $g(\mathbf{x})=a_{g}(\mathbf{x})$ $\cos \left(2 \pi \varphi_{g, 1}\left(x_{1}^{\prime}\right)\right) \cos \left(2 \pi \varphi_{g, 2}\left(x_{2}^{\prime}\right)\right)$ where $a_{g}(\mathbf{x}), \varphi_{g, 1}(\cdot)$ and $\varphi_{g, 2}(\cdot)$ are assumed to be slowly varying, defining $\varphi_{g, u}^{\prime}\left(x_{1}\right)=\frac{\partial}{\partial x_{1}} \varphi_{g, u}\left(x_{1}\right)$, for $u=1,2$, and additionally assuming $\varphi_{g, 1}^{\prime}\left(x_{1}\right)-\varphi_{g, 2}^{\prime}\left(x_{2}\right)=C$, a constant for all $x_{1}$ and $x_{2}$ such that $a_{g}(\mathbf{x})$ is non-negligible, is given with $\mathbf{b}^{\prime}=\boldsymbol{r}_{-\theta} \mathbf{b}$ by

$$
\begin{aligned}
w_{\psi}^{(++)}(\boldsymbol{\xi} ; g)= & a_{g}(\mathbf{b}) a \Psi^{(e e)}\left(a f_{g, 1}(\mathbf{b}, \theta), a f_{g, 2}(\mathbf{b}, \theta)\right)\left[\cos \left(2 \pi \varphi_{g, 1}\left(b_{1}^{\prime}\right)\right)\right) \cos \left(2 \pi \varphi_{g, 2}\left(b_{2}^{\prime}\right)\right) \\
& +\boldsymbol{i} \sin \left(2 \pi \varphi_{g, 1}\left(b_{1}^{\prime}\right)\right) \cos \left(2 \pi \varphi_{g, 2}\left(b_{2}^{\prime}\right)\right)+\boldsymbol{j} \cos \left(2 \pi \varphi_{g, 1}\left(b_{1}^{\prime}\right)\right) \sin \left(2 \pi \varphi_{g, 2}\left(b_{2}^{\prime}\right)\right) \\
& \left.+\boldsymbol{k} \sin \left(2 \pi \varphi_{g, 1}\left(b_{1}^{\prime}\right)\right) \sin \left(2 \pi \varphi_{g, 2}\left(b_{2}^{\prime}\right)\right)\right]+o(1) \\
w_{\psi}^{(++)}(\boldsymbol{\xi} ; g)= & a_{g}(\mathbf{b}) a \Psi^{(e e)}\left(a f_{g, 1}(\mathbf{b}, \theta), a f_{g, 2}(\mathbf{b}, \theta)\right) e^{2 \pi \boldsymbol{i} \varphi_{g, 1}\left(b_{1}^{\prime}\right)} e^{2 \pi \boldsymbol{j} \varphi_{g, 2}\left(b_{2}^{\prime}\right)}+o(1) .
\end{aligned}
$$

where $\boldsymbol{f}_{g}(\mathbf{x}, \theta)=\left[\varphi_{g, 1}^{\prime}\left(x_{1}^{\prime}\right), \quad \varphi_{g, 2}^{\prime}\left(x_{2}^{\prime}\right)\right]^{T}$. Note that the constraints imposed on $g(\mathbf{x})$ includes a local plane wave structure as then one of the two phase functions will itself be constant, and separable structure with different local periods, as this will be modelled via two different local plane waves separated to different values of $a$ and $\theta$.

Proof: The proof follows by direct calculation, if some care is taken with the conjugation and appropriate assumptions are made regarding the variability of the amplitude and phase functions. It is necessary to use a 
hypercomplexing wavelet for the formula to follow. Let $\mathbf{f}_{0}=\arg _{f_{1}>0} \max \left|\Psi^{(e e)}(\mathbf{f})\right|$. We note that a simplified description of the separable oscillatory signal $g(\mathbf{x})$ can be determined from the ridge of the CWT $c f$ [43][p. 394] via analysis for values of $\boldsymbol{\xi}$ in a subspace given by $\left\{\boldsymbol{\xi}: \boldsymbol{f}_{g}(\mathbf{b}, \theta)=a^{-1} \mathbf{f}_{0}\right\}$ such that the measure of the degree of separability of $w_{\psi}^{(++)}(\boldsymbol{\xi} ; g)$ is zero. Direct calculation shows that, when the CWT is calculated at another angle than that of the variation in $g(\mathbf{x})$, the magnitude of the wavelet transform is less than that of equation (101). The ridges, and thus $\boldsymbol{f}_{g}(\mathbf{b}, \theta)$, can be determined from maxima in $\left|w_{\psi}^{(++)}(\boldsymbol{\xi} ; g)\right|$, when the latter magnitude is calculated for fixed $a$ and $\mathbf{b}$ varying $\theta$, but to verify the chosen form of the separable orientation is appropriate we additionally calculate the polar representation of $w_{\psi}^{(++)}(\boldsymbol{\xi} ; g)$ and determine that indeed $\widetilde{\gamma}_{-\theta, \theta}(\mathbf{b})=0$, where the latter is the $\boldsymbol{k}$ angle in the polar representation of $w_{\psi}^{(++)}(\boldsymbol{\xi} ; g)$, see equation (28). Once $\theta^{\prime}$, has been determined we calculate $\widetilde{\alpha}_{-\theta^{\prime}, \theta^{\prime}}(\mathbf{b})$ and $\widetilde{\beta}_{-\theta^{\prime}, \theta^{\prime}}(\mathbf{b})$, these two functions then characterising the structure of the locally separable oscillation, where $\left|w_{\psi}^{(++)}(\boldsymbol{\xi} ; g)\right|$ corresponds to the magnitude of the oscillation. Signals corresponding to aggregations of several separable oscillatory components can be locally analysed as long as the wavelets are sufficiently concentrated in space and spatial frequency. For further notes on separating distinct components see [43][p. 395], where such methods mutatis mutandis can be applied in this context. The main focus in this article is not the development of additional wavelet ridge methods, but the expression in equation 101 shows the structural representation possible with the transform coefficients.

\section{Phase shifts in both orthogonal axes}

We define phase shifts in both directions of variation for separable signals. The shifted signal depends on the axes of the direction of variation specified via $\theta$, something that will not be the case for the single phase-shift defined via the monogenic structure.

Definition 4.2: Phase shift for a separable structure of orientation $\theta$.

For a real signal that is separable when viewed in the correct axes of observation, i.e. $g(\mathbf{x})$ satisfies equation (32), the signal phase shifted by $\boldsymbol{\theta}_{s}=\left(\begin{array}{ll}\theta_{s, 1} & \theta_{s, 2}\end{array}\right)^{T}$ is defined by:

$$
\begin{aligned}
\Lambda_{\boldsymbol{\theta}_{s}, \theta}^{2 D} g(\mathbf{x}) & \equiv\left|\widetilde{g}_{-\theta, \theta}^{(++)}(\mathbf{x})\right| \cos \left(2 \pi \widetilde{\alpha}_{-\theta, \theta}(\mathbf{x})-\theta_{s, 1}\right) \cos \left(2 \pi \widetilde{\beta}_{-\theta, \theta}(\mathbf{x})-\theta_{s, 2}\right) \\
& =\Re\left\{\left|\widetilde{g}_{-\theta, \theta}^{(++)}(\mathbf{x})\right| e^{\left(i\left(2 \pi \widetilde{\alpha}_{-\theta, \theta}(\mathbf{x})-\theta_{s, 1}\right)\right)} e^{\left(\boldsymbol{j}\left(2 \pi \widetilde{\beta}_{-\theta, \theta}(\mathbf{x})-\theta_{s, 2}\right)\right)}\right\},
\end{aligned}
$$

where $\Lambda_{\boldsymbol{\theta}_{s}, \theta}^{2 D}$ is denoted the phase shift operator. $\Lambda_{\boldsymbol{\theta}_{s}, \theta}^{2 D}$ shifts the two separable oscillations in cycle by $\theta_{s, 1}$ and $\theta_{s, 2}$, respectively.

Proposition 18: Hypercomplex signal of the phase-shifted separable signal.

For a real signal $g(\mathbf{x})$ satisfying equation (32) the $\theta$-hypercomplex extension of the phase shifted real image in direction $\theta$ is given by:

$$
\begin{aligned}
\left(\widetilde{\Lambda_{\boldsymbol{\theta}_{s}, \theta} g}\right)_{-\theta, \theta}^{(++)}(\mathbf{x}) & =\left|\widetilde{g}_{-\theta, \theta}^{(++)}(\mathbf{x})\right| e^{\left(i\left[2 \pi \widetilde{\alpha}_{-\theta, \theta}(\mathbf{x})-\theta_{s, 1}\right)\right]} e^{\left.\boldsymbol{j}\left[2 \pi \widetilde{\beta}_{-\theta, \theta}(\mathbf{x})-\theta_{s, 2}\right)\right]} \\
& =e^{-\boldsymbol{i} \theta_{s, 1}} \widetilde{g}_{-\theta, \theta}^{(+)}(\mathbf{x}) e^{-\boldsymbol{j} \theta_{s, 2}}
\end{aligned}
$$

Proof: See section ஹII-E

Theorem 6: HCWT of a phase-shifted separable signal.

The CWT of a phase-shifted signal, where $g(\mathbf{x})$ satisfies equation (32), using a hypercomplex wavelet is given by

$$
w_{\psi}^{(++)}\left(\boldsymbol{\xi} ; \Lambda_{\boldsymbol{\theta}_{s}, \theta}^{2 D} g\right)=e^{-\boldsymbol{i} \theta_{s, 1}} w_{\psi}^{(++)}(\boldsymbol{\xi} ; g) e^{-\boldsymbol{j} \theta_{s, 2}}
$$

Proof: See appendix ЩI-F

Corollary 1: Magnitude invariance of the HCWT of a separable signal under phase-shifts.

The magnitude of the CWT of a phase-shifted separable signal is equivalent to the CWT of the original signal.

Proof: From equation (104) we may note that $\Lambda_{\boldsymbol{\theta}_{s}, \theta}^{2 D} w^{(++)}(\boldsymbol{\xi} ; g)=e^{\boldsymbol{i} \theta_{s, 1}} w_{\psi}^{(++)}(\boldsymbol{\xi} ; g) e^{\boldsymbol{j} \theta_{s, 2}}$, and thus it follows that

$$
\left|w_{\psi}^{(++)}\left(\boldsymbol{\xi} ; \Lambda_{\boldsymbol{\theta}_{s}, \theta}^{2 D} g\right)\right|^{2}=\left|w_{\psi}^{(++)}(\boldsymbol{\xi} ; g)\right|^{2} .
$$


It thus follows that if the original image was separable then the magnitude of the CWT coefficients calculated at the correct orientation is stable with respect to phase-shifts. Given the dependence of the analysis on the orientation of the signal it is not surprising to find that the analysis strongly depends on the local orientation of the image. Our representation of the same image should change substantially if we assume separability in different directions. We may deduce that for signals that can be described as locally separable that small changes in the spatial alignment between the wavelets and the signal will not cause migration of energy over frequency bands. The result is important, as if discrete filter banks are constructed to implement the CWT then $\psi^{++}(\mathbf{x})$ phase-shift invariant in magnitude implies that the sampled wavelet coefficients will not suffer from substantial spatial shift variance, but there is still orientation dependence.

\section{The monogenic CWT}

The interpretability of the hypercomplex decomposition coefficients depends on the assumption of locally separable variation present in the image. To be able to retrieve the structural representation of the signal the appropriate axes for analysis have to be identified; this in general requires the calculation of the HCWT at the full set of $\theta \in[0, \pi]$, unless it transpires that the locally preferred orientation is already known. If at a fixed spatial location $\mathbf{x}=\mathbf{b}$ and given set of frequencies there was variation in only one orientation, the structure could be represented by the scale localised monogenic signal. If at any given spatial point $\mathbf{x}, g(\mathbf{x})$ corresponded to a single component, then the local characteristics of $g(\mathbf{x})$ could be extracted from $g^{( \pm)}(\mathbf{x})$, and the polar representation of equation (46) can be used directly to represent the signal. However, in general, $g(\mathbf{x})$ will correspond to a multiscale structure at the spatial point $\mathbf{x}$, and thus a scale local representation using the monogenic wavelets is necessary to implement in order to produce an interpretable polar representation. The monogenic CWT, or MCWT, represents local structure in terms of locally unidirectional variation, where the direction of variation can be determined from the transform coefficients at a single local point. In general, analysis with a monogenic, but not necessarily isotropic monogenic mother wavelet, is implemented i.e. a quaternionic mother wavelet of the form $\psi(\cdot)=\psi^{(+)}(\cdot)=\psi^{(r)}(\cdot)+\boldsymbol{i} \psi_{R}^{(1)}(\cdot)+\boldsymbol{j} \psi_{R}^{(2)}(\cdot)$ is used. A non-isotropic mother wavelet is a suitable choice as it is not reasonable to assume that the scale localisation alone will be sufficient to separate components present in the image. We use an isotropic mother wavelet, when, locally, there is only a single unidirectional oscillation present.

The HCWT were constructed to ensure that the coefficients rather than the wavelets had interpretable polar representation. Unfortunately, we found that these two requirements could not be simultaneously achieved. It will transpire for the monogenic wavelets that even if the wavelets are themselves $\theta$-monogenic, so are the wavelet coefficients, unlike the previous case. This all is due to the zero $\boldsymbol{k}$-component in the quaternionic representation. The coefficients are represented in terms of an amplitude, an orientation and a local period. Again, to be able to establish the properties of the monogenic coefficients, the transform in the Fourier domain is considered in detail. We show that the MCWT annihilates the anti-monogenic component, and this ensures that, equivalently, firstly the monogenic signal may be constructed and then scale localised; or firstly the image can be scale localised and then the monogenic extension of the local signal constructed. Indeed both operations can be implemented in one step using the MCWT, and this then establishes the interpretation of the wavelet coefficients.

Proposition 19: FT \& QFT of the MCWT.

The FT, and the QFT, of the MCWT of a real signal are given by:

$$
\begin{aligned}
W_{\psi}^{(+)}(\boldsymbol{\zeta} ; g) & =\left[1-\boldsymbol{k} \cos \left(\phi_{b}-\theta\right)+\sin \left(\phi_{b}-\theta\right)\right] W_{\psi}^{(r)}(\boldsymbol{\zeta} ; g) \\
W_{\psi ; Q}^{(+)}(\boldsymbol{\kappa} ; g) & =W_{\psi ; Q}^{(r)}(\boldsymbol{\kappa} ; g)+\cos \theta\left[\cos \nu_{b}+\sin \nu_{b}\right] W_{\psi ; Q}^{(r)}(\boldsymbol{\kappa} ; g)+\sin \theta\left[\cos \nu_{b}-\sin \nu_{b}\right] \boldsymbol{i} W_{\psi ; Q}^{(r)}(\boldsymbol{\kappa} ; g) \boldsymbol{j} .
\end{aligned}
$$

Proof: See section $\amalg I I-G$ From these equation the frequency domain properties of the monogenic wavelet coefficients can be determined. Note that from equations (106) and (42) we can immediately deduce that the MCWT coefficients are $\theta$-monogenic. If the real mother wavelet is radially symmetric, $\psi^{(r)}(\mathbf{x})=\psi^{(e)}(x)$, then the rotation has no important effect in the definition of CWT, [87). This assumption implies that $\Psi^{(e)}(f) \in \mathbb{R}$, and $a \Psi^{(e) *}\left(a \boldsymbol{r}_{-\theta} \mathbf{f}\right)=a \Psi^{(e)}(a f)$.

Corollary 2: FT and QFT of the isotropic MCWT. 
The FT, and the QFT, of the MCWT based on a real isotropic mother wavelet of a real signal is given by:

$$
\begin{aligned}
W_{\psi}^{(e)}(\boldsymbol{\zeta} ; g) & =\Psi^{(e)}\left(a f_{b}\right) G\left(\mathbf{f}_{b}\right), \\
W_{\psi}^{(+)}(\boldsymbol{\zeta} ; g) & =\left[1+\left(-\boldsymbol{k} \cos \left(\phi_{b}-\theta\right)+\sin \left(\phi_{b}-\theta\right)\right)\right] \Psi^{(e)}\left(a f_{b}\right) G\left(\mathbf{f}_{b}\right) \\
& =\Psi^{(+)}\left(a \boldsymbol{r}_{-\theta} \mathbf{f}_{b}\right) G\left(\mathbf{f}_{b}\right) \neq \Psi^{(+) *}\left(a \boldsymbol{r}_{-\theta} \mathbf{f}_{b}\right) G\left(\mathbf{f}_{b}\right) \\
& =\left[1+\left(-\boldsymbol{k} \cos \left(\phi_{b}-\theta\right)+\sin \left(\phi_{b}-\theta\right)\right)\right] W_{\psi}^{(e)}(\boldsymbol{\zeta} ; g) \\
W_{\psi ; Q}^{(+)}(\boldsymbol{\kappa} ; g) & =\left\{G_{Q}\left(\mathbf{q}_{b}\right)\left[1+\cos \theta\left(\cos \nu_{b}+\sin \nu_{b}\right)\right]+\sin \theta\left[\cos \nu_{b}-\sin \nu_{b}\right] \boldsymbol{i} G_{Q}(\mathbf{q}) \boldsymbol{j}\right\} a \Psi^{(e)}\left(a q_{b}\right) .
\end{aligned}
$$

These relations will enable us to consider the properties of the magnitude of the wavelet coefficients under rotation, and we wish to derive certain relations regarding the properties of the CWT in these circumstances.

Proposition 20: FT of the MCWT.

The FT of the MCWT of a monogenic extension of a real signal takes the form:

$$
W_{\psi}^{( \pm)}\left(\boldsymbol{\zeta} ; g^{(+)}\right)=\left[(1 \pm \cos (\theta)) G^{(+)}\left(\mathbf{f}_{b}\right) \pm \boldsymbol{k} \sin (\theta) G^{(-)}\left(\mathbf{f}_{b}\right)\right] a \Psi^{(r) *}\left(a \boldsymbol{r}_{-\theta} \mathbf{f}_{b}\right) .
$$

The anti-monogenic extension has an equivalent form with the \pm on the right hand side replaced by $\mp$.

Proof: See section $\llbracket-\mathrm{H}$ and set $\theta^{\prime}=0$.

Proposition 21: CWT of $\theta$-monogenic \& anti-monogenic decomposition components.

The Fourier transform of the MCWT of the extended $\theta$-monogenic and anti-monogenic extension of a real signal take the forms:

$$
\begin{aligned}
W_{\psi}^{(+)}\left(\boldsymbol{\zeta} ; \widetilde{g}_{-\theta, \theta}^{(+)}\right)= & \left\{2+2 \sin \left(\phi_{b}-\theta\right)-2 \boldsymbol{k} \cos \left(\phi_{b}-\theta\right)\right\} W_{\psi}^{(r)}(\boldsymbol{\zeta} ; g) \\
& =2 \widetilde{G}_{-\theta, \theta}^{(+)}\left(\mathbf{f}_{b}\right) a \Psi^{(r) *}\left(a \boldsymbol{r}_{-\theta} \mathbf{f}_{b}\right) . \\
= & 2 W_{\psi}^{(r)}\left(\boldsymbol{\zeta} ; \widetilde{g}_{-\theta, \theta}^{(+)}\right), \quad w_{\psi}^{(+)}\left(\boldsymbol{\xi} ; \widetilde{g}_{-\theta, \theta}^{(+)}\right)=2 w_{\psi}^{(r)}\left(\boldsymbol{\xi} ; \widetilde{g}_{-\theta, \theta}^{(+)}\right) \\
W_{\psi}^{(+)}\left(\boldsymbol{\zeta} ; \widetilde{g}_{-\theta \theta}^{(-)}\right)= & 0, \quad w_{\psi}^{(+)}\left(\boldsymbol{\xi} ; \widetilde{g}_{-\theta \theta}^{(-)}\right)=0
\end{aligned}
$$

Proof: See section

Hence the MCWT of a $\theta$-monogenic signal observed in the rotated frame of reference, i.e. $\widetilde{g}_{-\theta, \theta}^{(+)}(\mathbf{x})$ is twice that of the real wavelet transform of the $\theta$-monogenic signal $\widetilde{g}_{-\theta, \theta}(\mathbf{x})$. The MCWT of $\widetilde{g}_{-\theta, \theta}^{(-)}(\mathbf{x})$ is zero. Thus the monogenic wavelet annihilates the anti-monogenic component of the image, in perfect analogy with 1-D, but we may further generalise the result to consider any combination of monogenic/anti-monogenic wavelet and monogenic/anti-monogenic signal.

Corollary 3: Annihilation using Monogenic \& Anti-Monogenic Wavelets.

The MCWT annihilated the $\theta$-anti-monogenic image and the anti-monogenic CWT annihilated the $\theta$-monogenic image:

$$
W_{\psi}^{( \pm)}(\boldsymbol{\zeta} ; g)=\frac{1}{2} W_{\psi}^{( \pm)}\left(\boldsymbol{\zeta} ; \widetilde{g}_{-\theta, \theta}^{( \pm)}\right), \quad w_{\psi}^{( \pm)}(\boldsymbol{\xi} ; g)=\frac{1}{2} w_{\psi}^{( \pm)}\left(\boldsymbol{\xi} ; \widetilde{g}_{-\theta, \theta}^{( \pm)}\right) .
$$

For any other angle than $\theta \pm l \pi, l \in \mathcal{Z}, w_{\psi}^{( \pm)}(\boldsymbol{\xi} ; g)$ is a linear combination of $w_{\psi}^{( \pm)}\left(\boldsymbol{\xi} ; g_{-\theta, \theta}^{(+)}\right)$and $w_{\psi}^{( \pm)}\left(\boldsymbol{\xi} ; g g_{-\theta, \theta}^{(-)}\right)$.

Proof: Comparing equation (III-H) with equation (106) the first result follows directly: furthermore the annihilation follows mutatis mutandis from equation (A-33). Thus, when analysing a real signal, the MCWT can be used to annihilate the anti-monogenic components in the real image, which is in complete analogy to the $d=1$ analytic/antianalytic case, [56], [76]. See equations (6.8) to (6.10), and in particular the unnumbered equation over equation (6.10), of [56]. These show that the CWT of an $d=1$ real signal with respect to an analytic (anti-analytic) wavelet is equal to one half of the CWT of the analytic (anti-analytic) extension of the real signal with respect to the analytic (anti-analytic) wavelet. In other words equation (110) is a generalisation of the equation over equation (6.10) of [56]. This implies that both in $d=1$ and $d=2$, the CWT of the real signal or image has the same phase and one half of the modulus of the CWT of the analytically or monogenically extended signal or image.

Corollary 4: Annihilation of $\pi$-rotated component.

If we assume $\psi^{(r)}=\psi^{(e)}$, then with the additional notation of $\boldsymbol{\zeta}_{\pi}=[a, \theta+\pi, \boldsymbol{b}]^{T}$, it follows that:

$$
W_{\psi}^{( \pm)}\left(\boldsymbol{\zeta}_{\pi} ; g\right)=\frac{1}{2} W_{\psi}^{( \pm)}\left(\boldsymbol{\zeta}_{\pi} ; \widetilde{g}_{-\theta, \theta}^{(\mp)}\right), \quad w_{\psi}^{( \pm)}\left(\boldsymbol{\xi}_{\pi} ; g\right)=\frac{1}{2} w_{\psi}^{( \pm)}\left(\boldsymbol{\xi}_{\pi} ; \widetilde{g}_{-\theta, \theta}^{(\mp)}\right) \text {. }
$$


If an isotropic wavelet is used then the monogenic wavelet rotated by $\pi$ is identical to the anti-monogenic wavelet, see equation (77), and so the result is an immediate consequence of the previous proposition.

Theorem 7: Construction of the local $\theta$-monogenic signal.

The MCWT of a real image $g(\mathbf{x})$ is equivalent to the scale localisation of the $\theta$-monogenic extension of the signal and the $\theta$-monogenic extension of the scale-localised version of the image $g(\mathbf{x})$ :

$$
w_{\psi}^{(+)}(\boldsymbol{\xi} ; g)=\left[w_{\psi}^{(r)}\left(\boldsymbol{\xi}_{0} ; \widetilde{g}_{-\theta}\right)\right]_{\theta}^{(+)}=w_{\psi}^{(r)}\left(\boldsymbol{\xi} ; \widetilde{g}_{-\theta, \theta}^{(+)}\right)
$$

Proof: See appendix [II-1] Thus all three local descriptions, i.e. the MCWT of a real signal, the real CWT of a $\theta$-monogenic signal and the monogenic extension of a real CWT of a real signal of a real-valued signal may all be viewed as equivalent. The left hand side of equation (112) simply corresponds to the MCWT of the real signal, whilst the middle equation is the $\theta$-monogenic extension of the scale local signal $\widetilde{g}_{-\theta}(\mathbf{x})$, which corresponds to adding some suitable components to the real part of the CWT of $g(\mathbf{x})$. The right hand side of equation (112) corresponds to first forming the monogenic extension of the signal, and then scale-localising the extension to make the phase description of the signal interpretable. Hence the MCWT can, assuming a single component has been retrieved at local index $\xi$, be represented in polar form using equation (46) where the magnitude, phase and orientation are interpretable in terms of a local univariate variations and the $\theta$-monogenic representation. Furthermore if we may assume the signal corresponds to a local plane wave the following theorem specifies its representation.

Theorem 8: The MCWT of a plane oscillatory signal.

The MCWT of a single component separable oscillatory signal modelled by $g(\mathbf{x})=a_{g}(\mathbf{x}) \cos \left(2 \pi \varphi_{g}(\mathbf{x})\right)$ where $a_{g}(\mathbf{x})$, as well as $\varphi_{g}(\mathbf{x})$ are assumed to be slowly varying, is given by:

$$
w_{\psi}^{(+)}(\boldsymbol{\xi}, g)=a_{g}(\mathbf{b}) a\left|\Psi^{(r)}\left(a \boldsymbol{r}_{-\theta} \boldsymbol{f}_{g}(\mathbf{b})\right)\right| e^{2 \pi \boldsymbol{e}_{n_{g}(\mathbf{b}, \theta)}\left[\varphi_{g}(\mathbf{b})-\varphi_{\psi}\left(a \boldsymbol{r}_{-\theta} \boldsymbol{f}_{g}(\mathbf{b})\right)\right]}+o(1),
$$

where $\boldsymbol{f}_{g}(\mathbf{x})=\nabla \varphi_{g}(\mathbf{x})=f_{g}(\mathbf{x})\left[\cos \left(\phi_{g}(\mathbf{x})\right) \quad \sin \left(\phi_{g}(\mathbf{x})\right)\right]^{T}$, $\boldsymbol{n}_{g}(\mathbf{x}, \theta)=\left[\begin{array}{ll}\cos \left(\phi_{g}(\mathbf{x})-\theta\right) & \sin \left(\phi_{g}(\mathbf{x})-\theta\right)\end{array}\right]^{T}, \Psi^{(r)}(\mathbf{f})=\left|\Psi^{(r)}(\mathbf{f})\right| e^{-2 \pi j \varphi_{\psi}(\mathbf{f})}$, and note that Hermitian symmetry imposes $\left|\Psi^{(r)}(\mathbf{f})\right|=\left|\Psi^{(r)}(-\mathbf{f})\right|$ as well as $\varphi_{\psi}(\mathbf{f})=-\varphi_{\psi}(-\mathbf{f})$.

Proof: This result was shown for the special case of the isotropic Morse wavelets in Metikas \& Olhede [44], and follows by direct calculation. Let $\mathbf{f}_{0}=\arg _{f_{1}>0} \max \left|\Psi^{(r)}(\mathbf{f})\right|$. We note that a simplified description of the oscillatory signal $g(\mathbf{x})$ can be determined from the ridge of the CWT $c f$ [43][p. 394] via the subspace of the locality index given by $\left\{\boldsymbol{\xi}: \boldsymbol{f}_{g}(\mathbf{b})=a^{-1} \boldsymbol{r}_{\theta} \mathbf{f}_{0}\right\}$. Equation (113) can be used to characterise the oscillation at $\mathbf{x}=\mathbf{b}$ and if the signal would be more appropriately modelled as an aggregation of of oscillatory signals as long as they are sufficiently separated, i.e. as long as the wavelet is sufficiently narrow in space and spatial frequency to separate the different component. Further analysis of the signal such as that of Olhede and Metikas [44], [71], [77], using isotropic monogenic wavelets, can then be implemented for arbitrary monogenic wavelets. Using directional monogenic wavelets will allow us to analyse a larger class of signal as it is more reasonable to assume an image corresponds to a collection of plane-waves if we localise in scale, position and orientation.

\section{E. Phase shifts for Unidirectional Variation}

Section IV-A noted that the stability of the CWT coefficients under given affine transformations of the argument was important. For the HCWT we remarked that the coefficients exhibited desirable properties for phase-shifted separable structure. With the monogenic transform we can obtain desirable properties for an arbitrary phase-shifted signal, and in some instances obtain invariance under rotation. As the local structure is described as a plane wave with orientation determined from the signal under observation this enables us to derive the additional results. Furthermore the phase-shift is not dependent on the choice of axes: the monogenic wavelet is identifying the direction of variation from the observed image, and then the phase-shift is given in terms of this direction. This is not equivalent to the hypercomplex wavelet coefficient structure.

Proposition 22: Local magnitude invariance under rotation.

If the real mother wavelet is isotropic, then the MCWT coefficients of a real image $g(\mathbf{x})$ at $\boldsymbol{\xi}$ will have a magnitude invariant to the value of $\theta$. 
Proof: See section [II-J] Hence the magnitude of the wavelet coefficients does not depend on the rotation angle, and we may deduce the result. This implies that we are not sensitive to loss of magnitude due to local orientation misalignment. However, we would naturally like to also be able to retrieve the local orientation, from the MCWT.

Definition 4.3: A directional signal.

A real signal $g(\mathbf{x})$ is considered as one-dimensionally directional at frequency $\mathbf{f}$ if $\exists \phi_{0} \in[0, \pi)$ such that

$$
G(\mathbf{f})=\frac{\widetilde{G}(f)}{2}\left[\delta\left(\phi-\phi_{0}-\pi\right)+\delta\left(\phi-\phi_{0}\right)\right] .
$$

We shall in this article be analysing real images: as $g(\mathbf{x}) \in \mathbb{R}$ its FT is Hermitian $G^{*}(\mathbf{f})=G(-\mathbf{f})$, and thus necessitates having both a delta distribution component at $\phi_{0}$ and $\phi_{0}+\pi$.

Theorem 9: Directional selectivity of the MCWT.

If the real mother wavelet is isotropic, and the analysed signal $g(\mathbf{x})$ is directional over the support of the monogenic wavelet at $\boldsymbol{\xi}$ with directionality $\phi_{0}$, then with $\boldsymbol{\xi}^{\dagger}=\left[a, \phi_{0}, \boldsymbol{b}\right]^{T}$

$$
\left|w_{\psi}^{(1)}\left(\boldsymbol{\xi}^{\dagger} ; g\right)\right|^{2}=\left|w_{\psi}^{(1)}(\boldsymbol{\xi} ; g)\right|^{2}+\left|w_{\psi}^{(2)}(\boldsymbol{\xi} ; g)\right|^{2}, \quad \phi_{0}-\theta=\tan ^{-1}\left(\frac{w_{\psi}^{(2)}(\boldsymbol{\xi} ; g)}{w_{\psi}^{(1)}(\boldsymbol{\xi} ; g)}\right)
$$

Proof: See section $\llbracket$ Thus with an isotropic mother wavelet, the magnitude of the Riesz component wavelet coefficients is invariant to rotations, but is maximised in the first component if we rotate the wavelet to align with the directionality of the variations. Hence we may determine the local directionality of the signal from the monogenic isotropic wavelet coefficients.

1) Phase-shifted plane wave signals : For a plane wave present at a given point $\mathbf{x}$ we define the phase-shift operation as follows.

Definition 4.4: Phase-shift of a plane wave.

For a real signal $g(\mathbf{x})=\widetilde{g}_{-\theta}\left(\boldsymbol{r}_{-\theta} \mathbf{x}\right)$ we note from equation (45) that the image may for any rotation angle $\theta$ be written in sinusoidal form and the phase shifted by $\theta_{s}$ version of the signal is defined via:

$$
\begin{aligned}
\Lambda_{\theta_{s}} g(\mathbf{x}) & \equiv\left|g^{(+)}(\mathbf{x})\right| \cos \left(2 \pi \phi(\mathbf{x})-\theta_{s}\right)=\Re\left\{\left|g^{(+)}(\mathbf{x})\right| \exp \left(\left(2 \pi \phi(\mathbf{x})-\theta_{s}\right) \boldsymbol{e}_{\widetilde{\nu}_{-\theta, \theta}}(\mathbf{x})\right)\right\} \\
& =\frac{1}{2}\left(e^{-\boldsymbol{e}_{\tilde{\nu}_{-\theta, \theta}}(\mathbf{x}) \theta_{s}} g_{-\theta, \theta}^{(+)}(\mathbf{x})+e^{\boldsymbol{e}_{\widetilde{\nu}_{-\theta, \theta}}(\mathbf{x}) \theta_{s}} g_{-\theta, \theta}^{(-)}(\mathbf{x})\right),
\end{aligned}
$$

where $\Lambda_{\theta_{s}}$ is denoted the phase shift operator.

Proposition 23: The $\theta$-monogenic phase-shifted signal.

For a real signal $g(\mathbf{x})$ the $\theta$-monogenic extension of the phase shifted real image if $\widetilde{\nu}_{-\theta, \theta}(\mathbf{x})$ is varying sufficiently slowly, i.e. assuming $\widetilde{\nu}_{-\theta, \theta}(\mathbf{x})=\widetilde{\nu}_{-\theta, \theta}\left(\mathbf{x}_{0}\right)$ for all $\mathbf{x}$ such that $\left|\widetilde{g}_{-\theta, \theta}^{(++)}(\mathbf{x})\right|$ is of non-negligible magnitude, takes the form:

$$
\begin{aligned}
\left(\Lambda_{\theta_{s}} g\right)_{\theta}^{(+)}(\mathbf{x}) & =\left|\widetilde{g}_{-\theta, \theta}^{(++)}(\mathbf{x})\right|\left[\cos \left(2 \pi \widetilde{\phi}_{-\theta, \theta}(\mathbf{x})-\theta_{s}\right)+\boldsymbol{e}_{\widetilde{\nu}_{-\theta, \theta}}(\mathbf{x}) \sin \left(2 \pi \widetilde{\phi}_{-\theta, \theta}(\mathbf{x})-\theta_{s}\right)\right] \\
& =e^{-\boldsymbol{e}_{\widetilde{\nu}_{-\theta}, \theta}(\mathbf{x}) \theta_{s}} \widetilde{g}_{-\theta, \theta}^{(+)}(\mathbf{x}) .
\end{aligned}
$$

If $\boldsymbol{e}_{\widetilde{\nu}_{-\theta, \theta}}(\mathbf{x})=e_{\widetilde{\nu}_{-\theta, \theta}}$, i.e. the orientation is constant across the width of the image, then as the Fourier transform corresponds to a right-hand multiplication, we may note

$$
\mathcal{F}\left\{\left(\Lambda_{\theta_{s}} g_{-\theta, \theta}^{(+)}(\mathbf{x})\right\}=e^{-\boldsymbol{e}_{\tilde{\nu}_{-\theta, \theta}} \theta_{s}} \widetilde{G}_{-\theta, \theta}^{(+)}(\mathbf{f}) .\right.
$$

Theorem 10: MCWT of a phase-shifted signal.

The MCWT of a phase-shifted signal, where the orientation of the signal is constant over the width of the wavelet is given by

$$
w_{\psi}^{( \pm)}\left(\boldsymbol{\xi} ; \Lambda_{\theta_{s}} g\right)=e^{\mp 2 \pi \boldsymbol{e}_{\check{\nu}_{\theta}}(\boldsymbol{b}) \theta_{s}} w_{\psi}^{( \pm)}(\boldsymbol{\xi} ; g) .
$$

Proof: See section $\amalg$ Thi-L result implies that the phase shift between two images that locally correspond to plane waves may easily be determined by equation (119).

Corollary 5: Magnitude invariance of MCWT under phase-shifts.

The magnitude of the MCWT of a phase-shifted signal, when the local orientation of the signal is stable over the width of the wavelet is equivalent to that of the not phase-shifted signal. 
Proof: From equation (119) we may note that $w_{\psi, \theta_{s}}^{( \pm)}(\boldsymbol{\xi} ; g)=e^{2 \pi \boldsymbol{e}_{\nu_{\theta}}(\boldsymbol{b}) \theta_{s}} w_{\psi}^{( \pm)}(\boldsymbol{\xi} ; g)$, and thus it follows that

$$
\left|w_{\psi}^{( \pm)}\left(\boldsymbol{\xi} ; \Lambda_{\theta_{s}} g\right)\right|^{2}=\left|w_{\psi}^{( \pm)}(\boldsymbol{\xi} ; g)\right|^{2} \text {. }
$$

Hence the magnitude of the MCWT is invariant to shifts of phase. This implies that there will be no migration of energy across scales due to misalignment between the wavelet and the signal. This shows the stability of the MCWT to misalignment between the observed signal and the analysis filters.

\section{CONCLuSions}

In this paper we have defined and provided suitable 2-D extensions of 1-D analytic wavelet decompositions that we denoted hyperanalytic. We have constructed classes of 2-D mother wavelets, as well as specific examples of such functions that may be used to calculate hyperanalytic decomposition coefficients, and discussed properties of the hyperanalytic wavelets and wavelet coefficients in detail. We have stressed the importance of magnitude invariance of the coefficients to phase-shifts of the image, and have constructed wavelets yielding coefficients that may be thought of as 'locally hyperanalytic' to exhibit such invariance.

We argued that the design of 2-D hyperanalytic decompositions in continuous space must start by understanding 2-D hyperanalytic signals. Hyperanalytic signals are constructed as the appropriate limit of hyperanalytic functions. A hyperanalytic function is a set of functions satisfying any generalisation of the Cauchy-Riemann system. When considering analysis in dimensions higher than 1-D there is in general more than one such system, and so a choice of the hyperanalytic system must be made. We considered two different systems in 2-D, namely the monogenic and hypercomplex systems. We carefully distinguished between hyperanalytic signals (limits of hyperanalytic functions) and hyperanalytic functions in our discussion.

Once a choice of hyperanalytic has been made, the hyperanalytic signal had to be suitably merged into the CWT to yield a hyperanalytic CWT set of coefficients. Two additional problems of interest were then resolved. Firstly the rotation operator does not commute with the convolutions that combine to construct a hyperanalytic signal from a real-valued image. To determine the structure of rotated hyperanalytic objects in a suitable frame of reference, we defined the notion of $\theta$-hyperanalytic as a function that is a hyperanalytic signal in a rotated frame. This definition allows for the interpretation of the hyperanalytic wavelet coefficients in terms of local variational structure in a given orientation. Furthermore the monogenic and hypercomplex wavelet transforms were related to the local UQFT (a unidirectional oscillatory representation), as well as the local QFT (a separable oscillatory representation), of an image. We demonstrated that our choices of hyperanalytic wavelet decompositions thus correspond to the representation of local image structure as either a plane wave (locally 1-D structure) and a local version of the UQFT basis elements, or as a combination of locally separable structures with a natural axis of observation and a local version of the QFT basis elements.

Given a hyperanalytic signal is a three or four vector-valued object it admits a representation as a quaternionic function. The local energy of the real signal was described by the magnitude of the quaternion, whilst its local structure was described by some suitable phase function/s defined in terms of the quaternionic elements, thus parameterising the local structure of the image in terms of local magnitude, scales and orientation of variation. Different polar descriptions were chosen for each of the two $\theta$-hyperanalytic constructions, as each hyperanalytic definition is suited to particular forms of local signal variations, and the interpretation of the magnitude and phases were discussed.

Secondly, as we demonstrated, a decomposition constructed using a hyperanalytic wavelet does not in general, even for zero rotation of the mother wavelet, yield wavelet coefficients that are hyperanalytic extensions of realvalued wavelet coefficients. In fact the wavelet coefficients need not be the real wavelet decomposition of a signal that has been extended to a hyperanalytic object. We introduced the concept of an hyperanalyticizing wavelet, as a function constructing hyperanalytic wavelet coefficients. In 1-D the analytic wavelets localise the signal in scale and time simultaneously as yielding a local interpretable description of the signal in terms of the local amplitude and the phase of the signal at that scale. As both the operation of localisation and construction of an analytic object commute in 1-D (both are multiplications in terms of complex objects) this simultaneous operation is not problematic. This, we demonstrated, is not the case in 2-D (quaternionic objects in general do not commute and, in addition, the rotation and the construction of hyperanalytic images do not commute). We showed that the hyperanalytic coefficients were equivalent to either constructing the real wavelet decomposition of the real image and 
then calculating the $\theta$-hyperanalytic extension of the wavelet coefficients or to constructing the $\theta$-hyperanalytic signal and then constructing the real wavelet coefficients of this object. This gave the interpretation of the hyperanalytic wavelet coefficients, as the $\theta$-hyperanalytic signal of the local projection or equivalently the local projection of the $\theta$-hyperanalytic component. To be able to prove these properties we defined the decomposition of any realvalued image into $\theta$-hyperanalytic and $\theta$-anti-hyperanalytic objects, in direct analogue with the analytic/anti-analytic decomposition in 1-D [41].

We demonstrated that the hyperanalyticizing wavelet either eliminates, or converts, the anti-hyperanalytic objects into hyperanalytic objects. The hyperanalytic decomposition allows for the determination of the CWT coefficients of phase-shifted images, where the elimination/conversion of the anti-components is pivotal for easy parameterisation of the coefficients of the phase shifted signal in terms of the coefficients of the original signal. For both the monogenic and hypercomplex coefficients we retrieved magnitude invariance of the wavelet coefficients under phase-shifts, even if in the hypercomplex case some restrictions had to be placed on the observed signal. Magnitude invariance to phase shift is an important property when discretizing the implementation: the local representation will be sampled and so small shifts in space should not change the format of the representation substantially. In the monogenic case we additionally demonstrated local invariance of magnitude under rotations with certain classes of mother wavelets and proved directional selectivity of the quaternionic coefficients. The stability and other properties of the transform depend on constructing wavelet coefficients that are hyperanalytic: this justifies our careful development of the hyperanalytizing wavelets.

In 1-D, analytic wavelets are also used for the decomposition and characterisation of non-stationary oscillatory signals, using wavelet ridge techniques [4], [5]. Usage of a localisation method is necessary for the analysis of multi-component non-stationary oscillatory signals, as for such signals the phase and amplitude description of the local structure in the spatial domain is not informative [30]. In 2-D we showed that wavelet ridges for unidirectional and separable oscillatory signals can be determined from the monogenic and hypercomplex wavelet coefficients. The advantage of using quaternionic wavelets is that separable oscillations can easily be parameterised in both directions simultaneously, and the analysis of unidirectional oscillations is simplified as once components have been separated in phase space the orientation of the variation can be determined from the monogenic wavelet coefficients without calculating the transform at all values of $\theta$. Our work thus complements existing ridge analysis using complex wavelets in 2-D. Given all the components of the quaternionic wavelet can be argued to have the same spatial and spatial frequency localisation only a few of the wavelet coefficients of most deterministic image features will be of non-negligible magnitude, whilst most noise spreads across all coefficients. Thus the localisation also increases the signal-to-noise ratio of the signal in the coefficients that contain most of the signal presence, and this further facilitates analysis of noisy realisations.

Finally, even if general results are of interest, to be able to implement the CWT, explicit examples of mother wavelets must be provided for analysis. Existing discrete hyperanalyticizing wavelets [2] and [1] were given an interpretation in terms of the general framework developed in this paper. Furthermore we provided families of continuous wavelets that are hyperanalyticizing. We chose to base the wavelets on the 1-D and 2-D generalized Morse wavelets, that can be shown to enjoy optimal localisation in phase space: we provided five classes of wavelets, the hypercomplex Morse wavelet, the separable and isotropic hypercomplexing Morse wavelets, as well as the the directional and isotropic monogenic Morse wavelets.

The study and development of analytic 1-D filters have lead to the synthesis of a wealth of signal processing methodology and applications. Before the discrete implementation was developed, continuous analytic wavelet decompositions using analytic wavelets were introduced and championed, such as wavelet ridge methods, and local decompositions of multivariate time series. This paper demonstrates the potential for hyperanalytic (quaternionic) methods, and the representation of local 2-D structure in terms of quaternionic objects. It is anticipated that methods based on quaternionic wavelet coefficients hold many novel challenges and potential future developments.

\section{ACKNOWLEDGMENTS}

SO \& GM would like to thank Dr Frederik Simons for valuable discussions and SO would like to thank Professor Andrew Walden for introducing her to this research area. GM gratefully acknowledges financial support from EPSRC (UK). 


\section{REFERENCES}

[1] I. W. Selesnick, R. G. Baraniuk, and N. G. Kingsbury, "The dual-tree complex wavelet transform”, IEEE Signal Processing Magazine, vol. 22(6), pp. 123-51, 2005.

[2] W. L. Chan, H. Choi, and R. Baraniuk, "Directional hypercomplex wavelets for multidimensional signal analysis and processing", IEEE International Conference on Acoustics, Speech, and Signal Processing (ICASSP), Houston, TX, May 2004.

[3] A. Grossman and J. Morlet, "Decomposition of Hardy functions into square integrable wavelets of constant shape", SIAM J. Math. Anal., vol. 15, pp. 723-736, 1984.

[4] S. Mallat, A Wavelet Tour of Signal Processing, 2nd Edition, Academic Press, New York, 1999.

[5] N. Delprat, B. Escudié, P. Guillemain, R. Kronland-Martinet, Ph. Tchamitchian, and B. Torresani, "Asymptotic wavelet and Gabor analysis: Extraction of instantaneous frequencies", IEEE Trans. Inform. Theory, vol. 38, pp. 644-64, 1992.

[6] P. Auscher, "Il n'existe pas de bases d'ondelettes regulieres dans l'espace Hardy $h^{2}(\backslash)$ ", C. R. Acad Sci Paris, vol. 315, pp. 769-72, 1992.

[7] S. Barber and G. P. Nason, "Real nonparametric regression using complex wavelets", J. Roy. Stat. Soc. B, vol. 66, pp. 927-939, 2004.

[8] N. G. Kingsbury, "Image processing with complex wavelets", Philosophical Transactions of the Royal Society of London A, vol. 357, pp. $2543-2560,1999$.

[9] N. G. Kingsbury, "Complex wavelets for shift invariant analysis and filtering of signals", Applied and Computational Harmonic Analysis, vol. 10, pp. 234-253, 2001.

[10] I. W. Selesnick, "The design of approximate Hilbert transform pairs of wavelet bases", IEEE Trans. on Signal Processing, vol. 50, pp. 1144-1152, 2002.

[11] I. W. Selesnick, "Hilbert transform pairs of wavelet bases", IEEE Signal Processing Letters, vol. 8, pp. 170-173, 2001.

[12] J.-M. Lina and M. Mayrand, "Complex daubechies wavelets", Applied and Computational Harmonic Analysis, vol. 2, pp. 219-229, 1995.

[13] J.-P. Antoine, R. Murenzi, P. Vandergheynst, and S. T. Ali, Two-Dimensional Wavelets and their Relatives, Cambridge University Press, United Kingdom, 2004.

[14] J.-L. Starck, Image processing and data analysis - the multiscale approach, Cambridge University Press, Cambridge, United Kingdom, 1998.

[15] J. L. Starck, E. J. Candès, and D. L. Donoho, "The curvelet transform for image denoising”, IEEE Trans. Image Processing, vol. 11, pp. 670-84, 2002.

[16] E. le Pennec and S. Mallat, "Sparse geometric image representations", IEEE Trans. Image Processing, vol. 14, pp. 423-38, 2005.

[17] T. Bülow and G. Sommer, "Hypercomplex signals-a novel extension of the analytic signal to the multidimensional case", IEEE Trans. Signal Processing, vol. 49, pp. 2844, 2001.

[18] T. Bülow, Hypercomplex spectral signal representations for the Processing and Analysis of Images, PhD thesis, Christian Albrechts Universität, Kiel, Germany, 1999.

[19] W. L. Hwang, C. S. Lu, and P. C. Chung, "Segmentation of perspective textured planes through the ridges of continuous wavelet transform", J. Visual Communication and Image Representation, vol. 201-16, 2001.

[20] J.-P. Antoine, R. Murenzi, and P. Vandergheynst, "Directional wavelets revisited: Cauchy wavelets and symmetry detection in patterns", Appl.Comput.Harmon.Anal., vol. 6, pp. 314, 1999.

[21] J.-P. Antoine and R. Murenzi, “Two-dimensional directional wavelets and the scale-angle representation”, Signal Processing, vol. 52, pp. 259, 1996.

[22] R. A. Gopinath, "The phaselet transform - an integral redundancy near shift-invariant wavelet transform", IEEE Trans. Signal Processing, vol. 51, pp. 1792-1805, 2003.

[23] S. L. Hahn, Hilbert Transforms in Signal Processing, Artech House, Norwood, MA, USA, 1996.

[24] F. C. A. Fernandes, R. L. C. van Spaendonck, and C. S. Burrus, "A new framework for complex wavelet transforms", IEEE Trans. Signal Proc., vol. 51, pp. 1825, 2003.

[25] M. Felsberg and G. Sommer, "The monogenic signal", IEEE Trans. Signal Processing, vol. 49, pp. $3136,2001$.

[26] S. L. Hahn and K. M. Snopek, "Wigner distributions and ambiguity functions of 2-d quaternionic and monogenic signals", IEEE Transactions on Signal Processing, vol. 53, pp. 3111-28, 2005.

[27] S. L. Hahn, "Multidimensional complex signals with single-orthant spectra", Proc. IEEE, vol. 80, pp. $1287,1992$.

[28] M.B. Wakin, M.T. Orchard, R. G. Baraniuk, and V. Chandrasekaran, "Phase and magnitude perceptual sensitivities in nonredundant complex wavelet representations", the Thirty-Seventh Asilomar Conference on Signals, Systems and Computers 9-12 Nov. $2003,2003$.

[29] B. Boashash, "Estimating and interpreting the instantaneous frequency of a signal-part; part I: Fundamentals, II: Algorithms", Proc. of IEEE, vol. 80, pp. 519, 1992.

[30] L. Cohen, Time-Frequency Analysis, Prentice-Hall, New Jersey, 1995.

[31] W. Martin and P. Flandrin, "Wigner-Ville spectral analysis of nonstationary processes", IEEE Trans. Acoustics, Speech and Signal Proc., vol. 33, pp. 1461-70, 1985.

[32] M. Holschneider, Wavelets: an analysis tool, Oxford University Press, Oxford, 1995.

[33] S. C. Olhede and A. T. Walden, "Generalized Morse wavelets", IEEE Trans. Signal Processing, vol. 50, pp. $2661,2002$.

[34] S. C. Olhede and A. T. Walden, "The Hilbert spectrum via wavelet projections", Proc. R. Soc. Lond. A, vol. 460, pp. $955,2004$.

[35] I. Cohen, S. Raz, and D. Malah, "Eliminating interference terms in the Wigner distribution using extended libraries of bases", in IEEE Conference on Acoustics, Speech and Signal Processing, Munich, Germany, April, 1997, ICASSP, pp. 284 - 288.

[36] S. C. Olhede and A. T. Walden, “'analytic' wavelet thresholding”, Biometrika, vol. 91, pp. 955-73, 2004.

[37] E. M. Stein, Singular Integrals and Differentiability Properties of Functions, Princeton University Press, Princeton, New Jersey, 1970.

[38] E. M. Stein and G. Weiss, Fourier Analysis on Euclidean Spaces, Princeton University Press, Princeton, New Jersey, 1971.

[39] A. C. Dixon, "On the Newtonian potential", Quart. J. Math. Oxford Series, vol. 35, pp. 283-96, 1904. 
[40] M. N. Nabighian, "Towards a three-dimensional automatic interpretation of potential field data via generalized Hilbert transforms: fundamental relations", Geophysics, vol. 49, pp. 780-6, 1984.

[41] A. Papoulis, Signal Analysis, McGraw-Hill, New York, USA, 1977.

[42] W. L. Hwang, C. S. Lu, and P. C. Chung, "Shape from texture: Estimation of planar surface orientation through the ridge surfaces of continuous wavelet transform”, IEEE Transactions on Image Processing, vol. 7, pp. 773-780, 1998.

[43] C. Gonnet and B. Torresani, "Local frequency analysis with two-dimensional wavelet transform", Signal Processing, vol. 90, pp. 389-404, 1994.

[44] G. Metikas and S. C. Olhede, "Multiple monogenic Morse wavelets", Tech. Rep., Imperial College Statistics Section Report TR-05-02, arXiv math.ST/0511324 revised version to appear in IEEE Transactions on Signal Processing, 2005.

[45] W. L. Chan, H. Choi, and R. Baraniuk, "Coherent image processing using quaternion wavelets", Proceedings of SPIE - Volume 5914 Wavelets XI, Manos Papadakis and Andrew F. Laine and Michael A. Unser (ed), September 2005.

[46] W. L. Chan, H. Choi, and R. Baraniuk, "Quaternion wavelets for image analysis and processing", IEEE International Conference on Image Processing (ICIP), Singapore, October 2004.

[47] M. Felsberg and G. Sommer, "The monogenic scale-space: A unifying approach to phase-based image processing in scale-space", Journal of Mathematical Imaging and Vision, vol. 21, pp. 5, 2004.

[48] E. Bayro-Corrochano, "Multi-resolution image analysis using the quaternion wavelet transform", Numerical Algorithms, vol. 39, pp. 35-55, 2005.

[49] T. Bülow, D. Pallek, and G. Sommer, "Quaternionic Gabor filters for local structure classification”, ICPR’98, Brisbane, Australia, 1998.

[50] L. Traversoni, "Quaternion wavelets for moving volume representation”, IEEE International Conference on Information Visualisation, IV, London, UK, July 2001.

[51] L. Traversoni, "Quaternion wavelets on cagd”, IEEE International Conference on Information Visualisation, I, 27-29 Aug,, August 1997.

[52] J.-X. He and B. Yu, "Wavelet analysis of quaternion-valued time-series", International Journal of Wavelets, Multiresolution and Information Processing, vol. 3, pp. 233-46, 2005.

[53] J.-X. He and B. Yu, "Continuous wavelet transforms on the space $L^{2}(\mathbb{R}, \mathbb{H} ; d x)$ ", Applied Mathematics Letters, vol. 17, pp. 111-21, 2004.

[54] C. C. Hsieh, "Motion smoothing using wavelets", Journal of Intelligent \& Robotic Systems, vol. 35, pp. 157-169, 2002.

[55] C. C. Hsieh, "B-spline wavelet-based motion smoothing", Computers and Industrial Engineering, vol. 41, pp. 59-76, 2001.

[56] S. C. Olhede and A. T. Walden, "Polarization phase relationships via multiple Morse wavelets-part I:fundamentals", Proc. R. Soc. Lond. A, vol. 459, pp. 413, 2003.

[57] A. C. Bovik, "Analysis of multichannel narrow-band filters for image texture segmentation", IEEE Trans. Image Processing, vol. 39, pp. 2025-43, 1991.

[58] C. A. Deavours, "The quaternion calculus", Amer. Math. Monthly, vol. 80, pp. 995, 1973.

[59] A. Sudbery, "Quaternionic analysis", Math. Proc. Camb. Phil. Soc., vol. 85, pp. 199-225, 1979.

[60] S-C. Pei, J-J. Ding, and J-H. Chang, "Efficient implementation of quaternion fourier tranform, convolution, and correlation by 2-d complex FFT", IEEE Trans. Signal Processing, vol. 49, pp. 2783-97, 2001.

[61] W. R. Hamilton, Elements of Quaternions, vol. 1 \& 2, Chelsea, New York, USA, 1895.

[62] C. E. Moxey, S. J. Sangwine, and T. A. Ell, "Hypercomplex correlation techniques for vector images", IEEE Trans. Signal Processing, vol. 51, pp. 1941-53, 2003

[63] T. Bülow, D. Pallek, and G. Sommer, "Riesz transforms for the isotropic estimation of the local phase of Moire interferograms", in Proceedings of the 22nd Symposium of the German Pattern Recognition Society (DAGM), Kiel, Germany, 2000.

[64] B. Boashash, "Note on the use of the Wigner distribution for time-frequency signal analysis", IEEE Trans. on Acoustics, Speech, and Signal Processing, vol. 36, pp. 1518, 1988.

[65] R. J. Duffin, “Two-dimensional Hilbert transforms”, Proceedings of the American Mathematical Society, vol. 8, pp. 239-45, 1957.

[66] M. Felsberg, Low-Level Image Processing with the Structure Multivector, PhD thesis, Christian Albrechts Universität, Kiel, Germany, 2002.

[67] M. Felsberg and G. Sommer, "Structure multivector for local analysis of images", Technical Report 2001, Christian Albrechts Universität, Kiel, Germany, 2000.

[68] J.-P. Antoine, P. Vandergheynst, and R. Murenzi, “Two-dimensional directional wavelets in image processing”, Int.J. Imaging Syst. Technolo., vol. 7, pp. 152, 1996.

[69] M. Farge, ", Annu.Rev.Fluid Mech., vol. 24, pp. 395, 1992.

[70] P. Cerejeiras, M. Ferreira, and U. Kähler, "Monogenic wavelets over the unit ball", Zeitschrift für Analysis und ihre Anwendungen, vol. 24, pp. 841-52, 2005.

[71] G. Metikas and S. C. Olhede, "Wavelet coherence", in Image Processing: Algorithms and Systems, Neural Networks, and Machine Learning, SPIE, San Jose, California, January, 2006, SPIE, pp. 31-42.

[72] I. Daubechies and T. Paul, "Time-frequency localisation operators: A geometric phase space approach ii. the use of dilations and translations.", Inverse Problems, vol. 4, pp. 661-80, 1988.

[73] I. Daubechies, Ten lectures on wavelets, Society for Industrial \& Applied Mathematics, Philadelphia, USA, 1992.

[74] M. Bayram and R. G. Baraniuk, "Multiple window time-frequency analysis", IEEE-SP International Symposium on Time-Frequency and Time-Scale Analysis, Paris, France, June 1996.

[75] R. R. Coifman and D. L. Donoho, Wavelets and Statistics (Lecture Notes in Statistics, Volume 103), chapter Translation-invariant denoising, pp. 125-50, Springer-Verlag, New York: USA, 1995.

[76] S. C. Olhede and A. T. Walden, "Polarization phase relationships via multiple Morse wavelets-part II:data analysis", Proc. R. Soc. Lond. A, vol. 459, pp. 641, 2003. 
[77] G. Metikas and S. C. Olhede, "Multiple wavelet analysis of amplitude/frequency modulated images", in Proc. IEEE International Symposium on Signal Processing and Information Technology, Athens, Greece, December, 2005, ISSPIT, pp. 284 - 288.

[78] E. M. Stein and G. Weiss, "Generalizations of the Cauchy-Riemann equations and representations of the rotation group", Amer. $J$. Math., vol. 90, pp. 163, 1968.

[79] S. L. Hahn and K. M. Snopek, "On the frequency-domain definition of the monogenic signal", Report No.1, Warsaw University of Technology, 2004.

[80] N. W. McLachlan, Bessel Functions for Engineers, Clarendon Press, Oxford, 1955.

[81] I. S. Gradshteyn and I. M. Ryzhik, Table of Integrals, Series, and Products, Academic Press, New York, 2000.

[82] M. Abramowitz and I. Stegun, Handbook of Mathematical Functions, Dover, New York, 1970.

\section{APPENDIX I}

A. $Q F T$ of $g_{\theta}^{(++)}(\mathbf{x})$

\section{HYPERANALYTIC PROPERTIES}

Note that $G(\mathbf{f})$ commutes with $\boldsymbol{j}$ as it is a function of $\boldsymbol{j}$ only. We obtain:

$$
\begin{aligned}
\mathcal{F}_{\mathcal{Q}}\left\{g_{\theta}^{(++)}(\mathbf{x})\right\}= & \mathcal{F}_{\mathcal{Q}}\left\{g\left(\boldsymbol{r}_{-\theta} \mathbf{x}\right)\right\}+i \mathcal{F}_{\mathcal{Q}}\left\{g_{H}^{(1)}\left(\boldsymbol{r}_{-\theta} \mathbf{x}\right)\right\}+\mathcal{F}_{\mathcal{Q}}\left\{g_{H}^{(2)}\left(\boldsymbol{r}_{-\theta} \mathbf{x}\right)\right\} \boldsymbol{j}+\boldsymbol{i} \mathcal{F}_{\mathcal{Q}}\left\{g_{H}^{(3)}\left(\boldsymbol{r}_{-\theta} \mathbf{x}\right)\right\} \boldsymbol{j} \\
= & \frac{1-\boldsymbol{k}}{2} G\left(\boldsymbol{r}_{-\theta} \mathbf{q}\right)+\frac{1+\boldsymbol{k}}{2} G\left(\boldsymbol{r}_{-\theta} \boldsymbol{J}_{\pi / 2} \mathbf{q}\right)+\boldsymbol{i}\left(\frac{1-\boldsymbol{k}}{2} G_{H}^{(1)}\left(\boldsymbol{r}_{-\theta} \mathbf{q}\right)\right. \\
& \left.+\frac{1+\boldsymbol{k}}{2} G_{H}^{(1)}\left(\boldsymbol{r}_{-\theta} \boldsymbol{J}_{\pi / 2} \mathbf{q}\right)\right)+\left(\frac{1-\boldsymbol{k}}{2} G_{H}^{(2)}\left(\boldsymbol{r}_{-\theta} \mathbf{q}\right)+\frac{1+\boldsymbol{k}}{2} G_{H}^{(2)}\left(\boldsymbol{r}_{-\theta} \boldsymbol{J}_{\pi / 2} \mathbf{q}\right)\right) \boldsymbol{j} \\
& +\boldsymbol{i}\left(\frac{1-\boldsymbol{k}}{2} G_{H}^{(3)}\left(\boldsymbol{r}_{-\theta} \mathbf{q}\right)+\frac{1+\boldsymbol{k}}{2} G_{H}^{(3)}\left(\boldsymbol{r}_{-\theta} \boldsymbol{J}_{\pi / 2} \mathbf{q}\right)\right) \boldsymbol{j} \\
= & \frac{1-\boldsymbol{k}}{2}\left(1+\operatorname{sgn}\left(\left[\boldsymbol{r}_{-\theta} \mathbf{q}\right]_{1}\right)\right)\left(1+\operatorname{sgn}\left(\left[\boldsymbol{r}_{-\theta} \mathbf{q}\right]_{2}\right)\right) G\left(\boldsymbol{r}_{-\theta} \mathbf{q}\right) \\
& +\frac{1+\boldsymbol{k}}{2}\left(1+\operatorname{sgn}\left(\left[\boldsymbol{r}_{\theta} \mathbf{q}\right]_{1}\right)\right)\left(1+\operatorname{sgn}\left(\left[\boldsymbol{r}_{\theta} \mathbf{q}\right]_{2}\right)\right) G\left(\boldsymbol{r}_{-\theta} \boldsymbol{J}_{\pi / 2} \mathbf{q}\right)
\end{aligned}
$$

Furthermore, if we had started by defining $\mathbf{x}^{\prime}=\boldsymbol{r}_{-\theta} \mathbf{x}$ and then found the QFT in $\mathbf{x}^{\prime}$, with QFT variable $\mathbf{q}^{\prime}$ we would have obtained $\mathcal{F}_{\mathcal{Q}}\left\{g_{\theta}^{(++)}\right\}\left(\mathbf{q}^{\prime}\right)=\left(1+\operatorname{sgn}\left(\left[\mathbf{q}^{\prime}\right]_{1}\right)\right)\left(1+\operatorname{sgn}\left(\left[\mathbf{q}^{\prime}\right]_{2}\right)\right) G_{Q}\left(\mathbf{q}^{\prime}\right)$, and so in the rotated frame of reference the QFT is supported on positive quaternionic frequency only.

\section{B. The $\theta$-Monogenic Signal}

With $p(\mathbf{x}, y)$ and $q_{s}(\mathbf{x}, y)$ defined by equation (34) let us denote by operator notation the construction of a system defined from $g(\mathbf{x})$ satisfying the Riesz system of equations: $\mathcal{P} g(\mathbf{x}, y)=(p(\cdot, y) * * g(\cdot))(\mathbf{x})$, and $\mathcal{Q}_{s} g(\mathbf{x}, y)=$ $\left(q_{s}(\cdot, y) * * g(\cdot)\right)(\mathbf{x})$. When $u_{R, g}(\mathbf{x}, y)=\mathcal{P} g(\mathbf{x}, y)$, and $v_{R, g}^{(s)}(\mathbf{x}, y)=\mathcal{Q}_{s} g(\mathbf{x}, y)$, we denote $k_{R ; g}^{+}(\mathbf{x}, y)=u_{R, g}(\mathbf{x}, y)+$ $\boldsymbol{i} v_{R, g}^{(1)}(\mathbf{x}, y)+\boldsymbol{j} v_{R, g}^{(2)}(\mathbf{x}, y)=\mathcal{P} g(\mathbf{x})+\boldsymbol{i} \mathcal{Q}_{1} g(\mathbf{x})+\boldsymbol{j} \mathcal{Q}_{2} g(\mathbf{x})$, which for $y \rightarrow 0^{+}$in the upper halfspace reduces to the monogenic function $g^{(+)}(\mathbf{x})=g(\mathbf{x})+\boldsymbol{i} g_{R}^{(1)}(\mathbf{x})+\boldsymbol{j} g_{R}^{(2)}(\mathbf{x})$. Rotate the solution to form, $R_{\theta} \mathcal{P} g(\mathbf{x}, y)+\boldsymbol{i} R_{\theta} \mathcal{Q}_{1} g(\mathbf{x}, y)+\boldsymbol{j} R_{\theta} \mathcal{Q}_{2} g(\mathbf{x}, y)$. Rotations require some attention; they commute with the convolution with the Poisson kernel $\left[\mathcal{R}_{\theta}, \mathcal{P}\right]=0$, but not with operation of the convolution with the conjugate Poisson kernels $\left[R_{\theta}, \mathcal{Q}_{j}\right] \neq 0$, see [38]. In particular,

$$
\left(\begin{array}{lll}
R_{\theta} & \mathcal{Q}_{1} & g(\mathbf{x}, y) \\
R_{\theta} & \mathcal{Q}_{2} & g(\mathbf{x}, y)
\end{array}\right)=\boldsymbol{r}_{-\theta}\left(\begin{array}{cc}
\mathcal{Q}_{1} & R_{\theta} g(\mathbf{x}, y) \\
\mathcal{Q}_{2} & R_{\theta} g(\mathbf{x}, y)
\end{array}\right)=\boldsymbol{r}_{-\theta}\left(\begin{array}{cc}
\mathcal{Q}_{1} & g\left(\boldsymbol{r}_{-\theta} \mathbf{x}, y\right) \\
\mathcal{Q}_{2} & g\left(\boldsymbol{r}_{-\theta} \mathbf{x}, y\right)
\end{array}\right) .
$$

As noted by [78], the Riesz system is invariant under dilation, translation, and simultaneous rotation of $\left(x_{1}, x_{2}\right)$ and $\left(\mathcal{Q}_{1} g, \mathcal{Q}_{2} g\right)$ by the same angle. In other words, $\left.\mathrm{A}-2\right)$ is also a solution of the Riesz system, but this time defined in variable $\mathbf{x}^{\prime}=\boldsymbol{r}_{-\theta} \mathbf{x}$. For $y \rightarrow 0^{+}$, A-2 reduces to

$$
\begin{aligned}
& R_{\theta} g(\mathbf{x})+\boldsymbol{i}\left[\cos \theta \mathcal{R}_{1} R_{\theta} g(\mathbf{x})+\sin \theta \mathcal{R}_{2} R_{\theta} g(\mathbf{x})\right] \\
& +\boldsymbol{j} \quad\left[-\sin \theta \mathcal{R}_{1} R_{\theta} g(\mathbf{x})+\cos \theta \mathcal{R}_{2} R_{\theta} g(\mathbf{x})\right] .
\end{aligned}
$$

This can be recast as $R_{\theta} g(\mathbf{x})+\boldsymbol{i} R_{\theta} \mathcal{R}_{1} g(\mathbf{x})+\boldsymbol{j} R_{\theta} \mathcal{R}_{2} g(\mathbf{x})$, see [38] for the algebraic properties of the Riesz kernels, and in analogy to $\mathrm{A}-2$,

$$
\left(\begin{array}{lll}
\mathcal{R}_{\theta} & \mathcal{R}_{1} & g(\mathbf{x}) \\
\mathcal{R}_{\theta} & \mathcal{R}_{2} & g(\mathbf{x})
\end{array}\right)=\boldsymbol{r}_{-\theta}\left(\begin{array}{lll}
\mathcal{R}_{1} & \mathcal{R}_{\theta} & g(\mathbf{x}) \\
\mathcal{R}_{2} & \mathcal{R}_{\theta} & g(\mathbf{x})
\end{array}\right)=\boldsymbol{r}_{-\theta}\left(\begin{array}{ll}
\mathcal{R}_{1} & g\left(\boldsymbol{r}_{-\theta} \mathbf{x}\right) \\
\mathcal{R}_{2} & g\left(\boldsymbol{r}_{-\theta} \mathbf{x}\right)
\end{array}\right) .
$$


Therefore, if a given function $g^{(+)}(\mathbf{x})$ is a monogenic signal, i.e. the limit of a monogenic function, then the function generated by rotating its axis by $\theta$ is also a monogenic signal, i.e. it is the limit as $y \rightarrow 0^{+}$of a system of functions, satisfying the Riesz system in a rotated frame of reference. A key component in the functionality of the set-up is that $\left|\boldsymbol{r}_{-\theta} \mathbf{x}\right|=|\mathbf{x}|$. Hence the $\theta$-monogenic signal is aptly named. Mutatis mutandis the proofs extend to the anti-monogenic case.

\section{APPENDIX II}

PRoperties of QUATERNIONIC WAVELETS

\section{A. FT and QFT of the Monogenic Wavelets}

We note that

$$
\Psi_{\boldsymbol{\xi}, Q}^{(+)}=a e^{-2 i \pi \boldsymbol{i} q_{1} b_{1}}\left[\mathcal{F}_{Q} R_{\theta}\left\{\psi^{(r)}+\boldsymbol{i} \psi_{R}^{(1)}+\psi_{R}^{(2)} \boldsymbol{j}\right\}(a \mathbf{q})\right] e^{-2 \pi \boldsymbol{j} q_{2} b_{2}},
$$

as each real wavelet is transformed according to:

$$
\begin{aligned}
\Psi_{\boldsymbol{\xi} ; Q}^{(r)}(\mathbf{q}) & =\mathcal{F}_{Q} U_{\boldsymbol{\xi}}\{\psi\}(\mathbf{q}) \\
& =\int_{-\infty}^{\infty} \int_{-\infty}^{\infty} e^{-2 \pi i q_{1} x_{1}} \frac{1}{a} \psi^{(r)}\left(\boldsymbol{r}_{-\theta}\left(\frac{\mathbf{x}-\mathbf{b}}{a}\right)\right) e^{-2 \pi \boldsymbol{j} q_{2} x_{2}} d^{2} \mathbf{x} \\
& =\int_{-\infty}^{\infty} \int_{-\infty}^{\infty} e^{-2 \pi \boldsymbol{i}\left(q_{1} b_{1}+a q_{1} y_{1}\right)} a \psi^{(r)}\left(\boldsymbol{r}_{-\theta} \mathbf{y}\right) e^{-2 \pi \boldsymbol{j}\left(q_{2} b_{2}+q_{2} a y_{2}\right)} d^{2} \mathbf{y} \\
& =a e^{-2 \pi i q_{1} b_{1}} \mathcal{F}_{Q}\left\{\psi^{(r)}\left(\boldsymbol{r}_{-\theta} \mathbf{x}\right)\right\}(a \mathbf{q}) e^{-2 \pi \boldsymbol{j} q_{2} b_{2}} .
\end{aligned}
$$

Note that from [79] for $\psi(\mathbf{x}) \in \mathbb{R}$

$$
\Psi_{Q}(\mathbf{q})=\frac{1-\boldsymbol{k}}{2} \Psi(\mathbf{q})+\frac{1+\boldsymbol{k}}{2} \Psi\left(\left(-q_{1}, q_{2}\right)\right),
$$

and we shall use this property on numerous occasions. We find with $\widetilde{\psi}_{\theta}^{(r)}(\mathbf{x})=R_{\theta}\left\{\psi^{(r)}\right\}$ (x) that

$$
\mathcal{F}_{Q} R_{\theta}\left\{\psi^{(r)}\right\}(\mathbf{q})=\mathcal{F}_{Q}\left\{\widetilde{\psi}^{(r)}\right\}(\mathbf{q})=\frac{1-\boldsymbol{k}}{2} \widetilde{\Psi}_{\theta}^{(r)}(\mathbf{q})+\frac{1+\boldsymbol{k}}{2} \widetilde{\Psi}_{\theta}^{(r)}\left(\boldsymbol{J}_{\pi / 2} \mathbf{q}\right) .
$$

We also note that $\widetilde{\Psi}_{\theta}^{(r)}(\mathbf{q})=\Psi^{(r)}\left(\boldsymbol{r}_{-\theta} \mathbf{q}\right)$. Thus we find

$$
\begin{aligned}
\mathcal{F}_{Q} R_{\theta}\left\{\psi^{(r)}\right\}(\mathbf{q})= & \frac{1-\boldsymbol{k}}{2} \Psi^{(r)}\left(\boldsymbol{r}_{-\theta} \mathbf{q}\right)+\frac{1+\boldsymbol{k}}{2} \Psi^{(r)}\left(\boldsymbol{r}_{-\theta} \boldsymbol{J}_{\pi / 2} \mathbf{q}\right) \\
= & \frac{1-\boldsymbol{k}}{2}\left[\frac{1+\boldsymbol{k}}{2} \Psi_{Q}^{(r)}\left(\boldsymbol{r}_{-\theta} \mathbf{q}\right)+\frac{1-\boldsymbol{k}}{2} \Psi_{Q}^{(r)}\left(\boldsymbol{J}_{\pi / 2} \boldsymbol{r}_{-\theta} \mathbf{q}\right)\right] \\
& +\frac{1+\boldsymbol{k}}{2}\left[\frac{1+\boldsymbol{k}}{2} \Psi_{Q}^{(r)}\left(\boldsymbol{r}_{-\theta} \boldsymbol{J}_{\pi / 2} \mathbf{q}\right)+\frac{1-\boldsymbol{k}}{2} \Psi_{Q}^{(r)}\left(\boldsymbol{J}_{\pi / 2} \boldsymbol{r}_{-\theta} \boldsymbol{J}_{\pi / 2} \mathbf{q}\right)\right] \\
= & \frac{1}{2} \Psi_{Q}^{(r)}\left(\boldsymbol{r}_{-\theta} \mathbf{q}\right)-\frac{\boldsymbol{k}}{2} \Psi_{Q}^{(r)}\left(\boldsymbol{J}_{\pi / 2+\theta / 2} \mathbf{q}\right)+\frac{\boldsymbol{k}}{2} \Psi_{Q}^{(r)}\left(\boldsymbol{J}_{\pi / 2-\theta / 2} \mathbf{q}\right) \\
& +\frac{1}{2} \Psi_{Q}^{(r)}\left(\boldsymbol{r}_{\theta} \mathbf{q}\right) .
\end{aligned}
$$

Similarly to $\Psi_{\boldsymbol{\xi}, Q}^{(r)}(\mathbf{q})$ we can now derive the QFTs of the members of the families produced from the first and second Riesz components of the monogenic mother wavelet, $\Psi_{R, \boldsymbol{\xi}, Q}^{(1)}(\mathbf{q})$ and $\Psi_{R, \boldsymbol{\xi}, Q}^{(2)}(\mathbf{q})$, as these two functions are real, see also [79]. As before, the crucial step is to calculate the QFT of the rotated real wavelets, by combining equation (A-7) with equation (37). This yields:

$$
\begin{aligned}
\mathcal{F}_{Q} R_{\theta}\left\{\psi_{R}^{(1)}\right\}(\mathbf{q}) & =\left(-\frac{\boldsymbol{i}}{2}\right) \cos (\eta-\theta)\left[\Psi_{Q}^{(r)}\left(\boldsymbol{r}_{-\theta} \mathbf{q}\right)-\boldsymbol{k} \Psi_{Q}^{(r)}\left(\boldsymbol{J}_{\pi / 2+\theta / 2} \mathbf{q}\right)\right] \\
& +\left(-\frac{\boldsymbol{i}}{2}\right) \cos (\eta+\theta)\left[\boldsymbol{k} \Psi_{Q}^{(r)}\left(\boldsymbol{J}_{\pi / 2-\theta / 2} \mathbf{q}\right)+\Psi_{Q}^{(r)}\left(\boldsymbol{r}_{\theta} \mathbf{q}\right)\right]
\end{aligned}
$$




$$
\begin{aligned}
\mathcal{F}_{Q} R_{\theta}\left\{\psi_{R}^{(2)}\right\}(\mathbf{q}) & =\frac{1}{2}\left[\Psi_{Q}^{(r)}\left(\boldsymbol{r}_{-\theta} \mathbf{q}\right)-\boldsymbol{k} \Psi_{Q}^{(r)}\left(\boldsymbol{J}_{\pi / 2+\theta / 2} \mathbf{q}\right)\right](-\boldsymbol{j}) \sin (\eta-\theta) \\
& +\frac{1}{2}\left[\boldsymbol{k} \Psi_{Q}^{(r)}\left(\boldsymbol{J}_{\pi / 2-\theta / 2} \mathbf{q}\right)+\Psi_{Q}^{(r)}\left(\boldsymbol{r}_{\theta} \mathbf{q}\right)\right](-\boldsymbol{j}) \sin (\eta+\theta)
\end{aligned}
$$

Putting equations $\mathrm{A}-6$, $\mathrm{A}-7 \mathrm{~A}, \mathrm{~A}-8$, and $\mathrm{A}-9$ together, we get the QFT of a member of the family generated through translations, dilations, and rotations from the entire mother monogenic wavelet:

$$
\begin{aligned}
\Psi_{\boldsymbol{\xi}, Q}^{(+)}(\boldsymbol{q}) & =\frac{a}{2} e^{-2 \pi \boldsymbol{i} q_{1} b_{1}}\left[(1+\cos (\eta-\theta)+\sin (\eta-\theta))\left(\Psi_{Q}^{(r)}\left(\boldsymbol{r}_{-\theta} \mathbf{q}\right)-\boldsymbol{k} \Psi_{Q}^{(r)}\left(\boldsymbol{J}_{\pi / 2+\theta / 2} \mathbf{q}\right)\right)\right. \\
& \left.+(1+\cos (\eta+\theta)+\sin (\eta+\theta))\left(\Psi_{Q}^{(r)}\left(\boldsymbol{r}_{\theta} \mathbf{q}\right)+\boldsymbol{k} \Psi_{Q}^{(r)}\left(\boldsymbol{J}_{\pi / 2-\theta / 2} \mathbf{q}\right)\right)\right] e^{-2 \pi \boldsymbol{j} q_{2} b_{2}}
\end{aligned}
$$

\section{B. The Wavelet Family of the Isotropic Monogenic Mother Wavelet}

From equation (74) we may note that the FT of a translated, dilated, and rotated monogenic wavelet which originates in a monogenic mother wavelet with radially symmetric real part respectively is given by:

$$
\begin{aligned}
\Psi_{\boldsymbol{\xi}}^{(+)}(\mathbf{f}) & =\mathcal{F} \psi_{\boldsymbol{\xi}}^{(+)}(\mathbf{x}) \\
& =\{1+[-\boldsymbol{k} \cos (\phi-\theta)+\sin (\phi-\theta)]\} a \Psi^{(e)}(a f) e^{-\boldsymbol{j} 2 \pi \mathbf{f}^{T} \mathbf{b}}, \\
\psi_{\boldsymbol{\xi}}^{(+)}(\mathbf{f}) & =\int_{-\infty}^{\infty} \int_{-\infty}^{\infty}\{1+[-\boldsymbol{i} \boldsymbol{j} \cos (\phi-\theta)+\sin (\phi-\theta)]\} a \Psi^{(e)}(a f) e^{-\boldsymbol{j} 2 \pi \mathbf{f}^{T}(\mathbf{b}-\mathbf{x})} d^{2} \mathbf{x} \\
& =\psi_{\boldsymbol{\xi}_{0}}^{(e)}(\mathbf{x})+\boldsymbol{i}\left[\cos (\theta) \psi_{R, \boldsymbol{\xi}_{0}}^{(1)}(\mathbf{x})+\sin (\theta) \psi_{R, \boldsymbol{\xi}_{0}}^{(2)}(\mathbf{x})\right]+\boldsymbol{j}\left[-\sin (\theta) \psi_{R, \boldsymbol{\xi}_{0}}^{(1)}(\mathbf{x})+\cos (\theta) \psi_{R, \boldsymbol{\xi}_{0}}^{(2)}(\mathbf{x})\right] .
\end{aligned}
$$

This gives the stated results. For the QFT expression, using equation (75), we note from equation (A-10), recalling that the QFT of an isotropic real wavelet is a real-valued object:

$$
\begin{aligned}
\Psi_{Q, \boldsymbol{\xi}}^{(+)}= & \frac{a}{2} e^{-2 \pi \boldsymbol{i} q_{1} b_{1}}\left[(1+\cos (\eta-\theta)+\sin (\eta-\theta))\left(\Psi_{Q}^{(e)}(q)-\boldsymbol{k} \Psi_{Q}^{(e)}(q)\right)\right. \\
& \left.+(1+\cos (\eta+\theta)+\sin (\eta+\theta))\left(\Psi_{Q}^{(e)}(q)+\boldsymbol{k} \Psi_{Q}^{(e)}(q)\right)\right] e^{-2 \pi \boldsymbol{j} q_{2} b_{2}} \\
= & a e^{-2 \pi \boldsymbol{i} q_{1} b_{1}} \Psi_{Q}^{(e)}(q)(1+\cos (\theta)(\cos (\eta)+\sin (\eta))+\boldsymbol{k} \sin (\theta)(-\sin (\eta)+\cos (\eta))) e^{-2 \pi j q_{2} b_{2}} .
\end{aligned}
$$

This yields the desired result.

\section{Directional Monogenic Wavelets}

We recall that

$$
\Psi_{1 D}^{(o)}(f)=-j \Psi_{1 D}^{(e)}(f), \quad \text { if } \quad f>0, \quad \Psi_{1 D}^{(o)}(f)=j \Psi_{1 D}^{(e)}(f), \quad \text { if } \quad f>0 .
$$

Direct calculation yields the result

$$
\begin{aligned}
\mathcal{R}_{1}\left\{\psi_{D}\right\}(\mathbf{x})= & \int_{-\infty}^{\infty} \int_{-\infty}^{\infty} \frac{f_{1}}{\boldsymbol{j} f}\left[\Psi_{1 D}^{(e)}\left(\frac{f_{1}-f_{2}}{\sqrt{2}}\right) \Psi_{1 D}^{(e)}\left(\frac{f_{1}+f_{2}}{\sqrt{2}}\right)-\Psi_{1 D}^{(o)}\left(\frac{f_{1}-f_{2}}{\sqrt{2}}\right) \Psi_{1 D}^{(o)}\left(\frac{f_{1}+f_{2}}{\sqrt{2}}\right)\right] \\
& \times e^{2 \mathbf{j} \pi \mathbf{f}^{T} \mathbf{x}} d^{2} \mathbf{f} \\
= & 4 \int_{0}^{\infty} \int_{0}^{\infty} \frac{g_{1}+g_{2}}{\sqrt{2} g}\left[\Psi_{1 D}^{(e)}\left(g_{1}\right) \Psi_{1 D}^{(e)}\left(g_{2}\right)\right] \sin \left(2 \pi \mathbf{g}^{T} \boldsymbol{r}_{\pi / 4} \mathbf{x}\right) d^{2} \mathbf{g} \\
= & 4 \int_{0}^{\infty} \int_{0}^{\infty} \frac{\sqrt{2} g_{1}}{g}\left[\Psi_{1 D}^{(e)}\left(g_{1}\right) \Psi_{1 D}^{(e)}\left(g_{2}\right)\right] \sin \left(2 \pi x_{1}\left(\frac{g_{1}+g_{2}}{\sqrt{2}}\right)\right) d^{2} \mathbf{g} .
\end{aligned}
$$

Thus $\mathcal{R}_{1}\left\{\psi_{D}\right\}(\mathbf{x})$ is constant in $x_{2}$ and odd in $x_{1}$, and a highly directional wavelet. We can in a similar fashion calculate the second Riesz transform to retrieve.

$$
\mathcal{R}_{2}\left\{\psi_{D}\right\}(\mathbf{x})=\frac{1}{\sqrt{2}}\left[\psi_{D}^{(\pi / 4,1)}\left(\boldsymbol{r}_{\pi / 4} \mathbf{x}\right)-\psi_{D}^{(\pi / 4,2)}\left(\boldsymbol{r}_{\pi / 4} \mathbf{x}\right)\right]=0 .
$$


Furthermore note that:

$$
\begin{aligned}
\psi_{D}(\mathbf{x})= & \int_{-\infty}^{\infty} \int_{-\infty}^{\infty}\left[\Psi_{1 D}^{(e)}\left(\frac{f_{1}-f_{2}}{\sqrt{2}}\right) \Psi_{1 D}^{(e)}\left(\frac{f_{1}+f_{2}}{\sqrt{2}}\right)-\Psi_{1 D}^{(o)}\left(\frac{f_{1}-f_{2}}{\sqrt{2}}\right) \Psi_{1 D}^{(o)}\left(\frac{f_{1}+f_{2}}{\sqrt{2}}\right)\right] \\
& \times e^{2 \boldsymbol{j} \pi \mathbf{f}^{T} \mathbf{x}} d^{2} \mathbf{f} \\
= & 4 \int_{0}^{\infty} \int_{0}^{\infty}\left[\Psi_{1 D}^{(e)}\left(g_{1}\right) \Psi_{1 D}^{(e)}\left(g_{2}\right)\right] \cos \left(2 \pi \mathbf{g}^{T} \boldsymbol{r}_{\pi / 4} \mathbf{x}\right) d^{2} \mathbf{g} .
\end{aligned}
$$

\section{Exact form of the spatial representation of the isotropic Morse Wavelets}

To be able to calculate the spatial domain representation of $\psi_{I}^{(+)}(\mathbf{x})$, given by equation 84 in the instance of the Morse wavelets, we make use of the following integrals, see [80],

$$
\int_{0}^{2 \pi} d \phi e^{\boldsymbol{j} z \cos (\theta-\phi)} \sin m \phi=2 \pi \boldsymbol{j}^{m} J_{m}(z) \sin m \theta, \quad \int_{0}^{2 \pi} d \phi e^{\boldsymbol{j} z \cos (\theta-\phi)} \cos m \phi=2 \pi \boldsymbol{j}^{m} J_{m}(z) \cos m \theta .
$$

In special cases $n=0, l>-2, m=1,2$ the analytic forms corresponding to equations (83) and (84) can be found. Note that $\mathrm{L}_{0}^{c}(x)=1$. For $m=1$ and $n=0$ we get

$$
\psi_{0 ; l, 1}^{(e)}(x)=\frac{A_{0, l, 1}^{\prime}}{2 \pi} \frac{1}{\left[1+x^{2}\right]^{\frac{l+2}{2}}} \Gamma(l+2) P_{l+1}^{0}\left(\frac{1}{\sqrt{1+x^{2}}}\right)
$$

where we have used equation 6.621 .1 of [81] and $P_{\nu}^{\mu}$ are the Legendre functions of degree $\nu$ and order $\mu$, see e.g [82]. The monogenic wavelet is

$$
\begin{aligned}
\psi_{0 ; l, 1}^{(+)}(\mathbf{x}) & =\psi_{0 ; l, 1}^{(e)}(x)+(\boldsymbol{i} \cos \chi+\boldsymbol{j} \sin \chi) \frac{A_{0, l, 1}^{\prime}}{2 \pi} \\
& \times \frac{1}{\left[1+x^{2}\right]^{\frac{l+2}{2}}} \Gamma(l+3) P_{l+1}^{-1}\left(\frac{1}{\sqrt{1+x^{2}}}\right) .
\end{aligned}
$$

For $m=2$ we get:

$$
\psi_{0 ; l, 2}^{(e)}(x)=\frac{A_{0, l, 2}^{\prime}}{2 \pi} \frac{1}{2} \Gamma\left(\frac{l+2}{2}\right){ }_{1} F_{1}\left(\frac{l+2}{2} ; 1 ;-\frac{x^{2}}{4}\right)
$$

where we have used equation 6.631 of [81]. For $l=2, s=1 / 2$, this wavelet is identical with the mexican hat, see e.g. [21]. The monogenic wavelet is:

$$
\begin{aligned}
\psi_{0 ; l, 2}^{(+)}(\mathbf{x}) & =\psi_{0 ; l, 2}^{(e)}(x)+(\boldsymbol{i} \cos \chi+\boldsymbol{j} \sin \chi) \frac{A_{0, l, 2}^{\prime}}{2 \pi} \\
& \times \frac{x}{4} \Gamma\left(\frac{l+3}{2}\right){ }_{1} F_{1}\left(\frac{l+3}{2} ; 2 ; \frac{-x^{2}}{4}\right) .
\end{aligned}
$$

\section{APPENDIX III}

\section{PROPERTIES OF THE QUATERNIONIC WAVELET COEFFICIENTS}

\section{A. QFT of the wavelet coefficients with an arbitrary quaternionic wavelet}

The CWT with respect to a quaternionic wavelet is written in terms of its real components and calculate the QFT component by component as follows:

$$
\begin{aligned}
\mathcal{F}_{Q}\left\{w_{\psi}(\boldsymbol{\xi} ; g)\right\} & =\mathcal{F}_{Q}\left\{w_{\psi}^{(r)}(\boldsymbol{\xi} ; g)\right\}-\boldsymbol{i} \mathcal{F}_{Q}\left\{w_{\psi}^{(i)}(\boldsymbol{\xi} ; g)\right\}-\mathcal{F}_{Q}\left\{w_{\psi}^{(j)}(\boldsymbol{\xi} ; g)\right\} \boldsymbol{j}-\boldsymbol{i} \mathcal{F}_{Q}\left\{w_{\psi}^{(k)}(\boldsymbol{\xi} ; g)\right\} \boldsymbol{j} \\
& =\frac{1}{2} W_{\psi}(\boldsymbol{\kappa} ; g)-\frac{\boldsymbol{k}}{2} W_{\psi^{(r)}-i \psi^{(i)}+j \psi^{(j)}-k \psi^{(k)}}(\boldsymbol{\kappa} ; g)+\frac{1}{2} W_{\psi}\left(\boldsymbol{J}_{\pi / 2} \boldsymbol{\kappa} ; g\right) \\
& +\frac{\boldsymbol{k}}{2} W_{\psi^{(r)}-i \psi^{(i)}+j \psi^{(j)}-k \psi^{(k)}}\left(\boldsymbol{J}_{\pi / 2} \boldsymbol{\kappa} ; g\right) \\
& \neq \frac{1-\boldsymbol{k}}{2} W_{\psi}(\boldsymbol{\kappa} ; g)+\frac{1+\boldsymbol{k}}{2} W_{\psi}\left(\boldsymbol{J}_{\pi / 2} \boldsymbol{\kappa} ; g\right) .
\end{aligned}
$$




\section{B. The Fourier Transform of the Hypercomplex wavelet transform of the $\theta$-Hypercomplex signal}

To establish the properties of the wavelet transform, we find the Fourier decomposition of the real signal in terms of the $\theta$-hypercomplex and anti-hypercomplex components. We note that for $\mu_{1}= \pm$, and $\mu_{2}= \pm$,

$$
\begin{aligned}
w_{\psi}^{\left(\mu_{1} \mu_{2}\right)}\left(\boldsymbol{\xi} ; \widetilde{g}_{-\theta^{\prime}, \theta^{\prime}}^{(++)}\right) & =w_{\psi}^{\left(\mu_{1} \mu_{2}\right)}\left(\boldsymbol{\xi} ; \widetilde{g}_{-\theta^{\prime}, \theta^{\prime} ; H}\right)+\boldsymbol{i} w_{\psi}^{\left(\mu_{1} \mu_{2}\right)}\left(\boldsymbol{\xi} ; \widetilde{g}_{-\theta^{\prime}, \theta^{\prime} ; H}^{(1)}\right)+\boldsymbol{j} w_{\psi}^{\left(\mu_{1} \mu_{2}\right)}\left(\boldsymbol{\xi} ; \widetilde{g}_{-\theta^{\prime}, \theta^{\prime} ; H}^{(2)}\right) \\
& +\boldsymbol{k} w_{\psi}^{\left(\mu_{1} \mu_{2}\right)}\left(\boldsymbol{\xi} ; \widetilde{g}_{-\theta^{\prime}, \theta^{\prime} ; H}^{(3)}\right) .
\end{aligned}
$$

Of course for any real-valued function $h(\cdot)$ by the linearity of the wavelet transform:

$$
w_{\psi}^{\left(\mu_{1} \mu_{2}\right)}(\boldsymbol{\xi} ; h)=w_{\psi}^{(e e)}(\boldsymbol{\xi} ; h)-\mu_{1} \boldsymbol{i} w_{\psi}^{(o e)}(\boldsymbol{\xi} ; h)-\mu_{2} \boldsymbol{j} w_{\psi}^{(e o)}(\boldsymbol{\xi} ; h)+\mu_{1} \mu_{2} \boldsymbol{k} w_{\psi}^{(o o)}(\boldsymbol{\xi} ; h) .
$$

Thus

$$
\begin{aligned}
W_{\psi}^{(++)}(\boldsymbol{\zeta} ; h)= & W_{\psi}^{(e e)}(\boldsymbol{\zeta} ; h)-\boldsymbol{i} W_{\psi}^{(e e)}(\boldsymbol{\zeta} ; h) \boldsymbol{j} \operatorname{sgn}\left(\left[\boldsymbol{r}_{-\theta} \mathbf{f}_{b}\right]_{1}\right)-\boldsymbol{j} W_{\psi}^{(e e)}(\boldsymbol{\zeta} ; h) \boldsymbol{j} \operatorname{sgn}\left(\left[\boldsymbol{r}_{-\theta} \mathbf{f}_{b}\right]_{2}\right) \\
& +\boldsymbol{k} W_{\psi}^{(e e)}(\boldsymbol{\zeta} ; h)(-1) \operatorname{sgn}\left(\left[\boldsymbol{r}_{-\theta} \mathbf{f}_{b}\right]_{1}\right) \operatorname{sgn}\left(\left[\boldsymbol{r}_{-\theta} \mathbf{f}_{b}\right]_{2}\right) \\
= & {\left[1+\operatorname{sgn}\left(\left[\boldsymbol{r}_{-\theta} \mathbf{f}_{b}\right]_{2}\right)-\boldsymbol{k} \operatorname{sgn}\left(\left[\boldsymbol{r}_{-\theta} \mathbf{f}_{b}\right]_{1}\right)-\boldsymbol{k} \operatorname{sgn}\left(\left[\boldsymbol{r}_{-\theta} \mathbf{f}_{b}\right]_{1}\right) \operatorname{sgn}\left(\left[\boldsymbol{r}_{-\theta} \mathbf{f}_{b}\right]_{2}\right)\right] W_{\psi}^{(e e)}(\boldsymbol{\zeta} ; h) } \\
= & {\left[1+\operatorname{sgn}\left(\left[\boldsymbol{r}_{-\theta} \mathbf{f}_{b}\right]_{2}\right)\right]\left[1-\boldsymbol{k} \operatorname{sgn}\left(\left[\boldsymbol{r}_{-\theta} \mathbf{f}_{b}\right]_{1}\right)\right] W_{\psi}^{(e e)}(\boldsymbol{\zeta} ; h) . }
\end{aligned}
$$

Thus the FT of the CWT of the hypercomplex image with respect to the hypercomplexing wavelet is given by:

$$
\begin{aligned}
W_{\psi}^{(++)}\left(\boldsymbol{\zeta} ; \widetilde{g}_{-\theta^{\prime}, \theta^{\prime}}^{\left(\mu_{1} \mu_{2}\right)}\right) & =W_{\psi}^{(++)}\left(\boldsymbol{\zeta} ; \widetilde{g}_{-\theta^{\prime}, \theta^{\prime}}\right)+\mu_{1} \boldsymbol{i} W_{\psi}^{(++)}\left(\boldsymbol{\zeta} ; \widetilde{g}_{-\theta^{\prime}, \theta^{\prime} ; H}^{(1)}\right)+\mu_{2} \boldsymbol{j} W_{\psi}^{(++)}\left(\boldsymbol{\zeta} ; \widetilde{g}_{-\theta^{\prime}, \theta^{\prime} ; H}^{(2)}\right) \\
& +\mu_{1} \mu_{2} \boldsymbol{k} W_{\psi}^{(++)}\left(\boldsymbol{\zeta} ; \widetilde{g}_{-\theta^{\prime}, \theta^{\prime} ; H}^{(3)}\right) .
\end{aligned}
$$

If we consider signals that are naturally aligned with the wavelet $\psi_{\boldsymbol{\xi}}^{++}(\cdot)$, i.e. take $\theta^{\prime}=\theta$, then a tedious but straightforward calculation yields

$$
W_{\psi}^{(++)}\left(\boldsymbol{\zeta} ; \widetilde{g}_{-\theta, \theta}^{(++)}\right)=2\left[1+\operatorname{sgn}\left(\left[\boldsymbol{r}_{-\theta} \mathbf{f}_{b}\right]_{2}\right)\right]\left[1-\boldsymbol{k} \operatorname{sgn}\left(\left[\boldsymbol{r}_{-\theta} \mathbf{f}_{b}\right]_{1}\right)\right] W_{\psi}^{(e e)}(\boldsymbol{\zeta}, g) .
$$

This quantity is non-zero in the first quadrant of the QFT domain, when $\theta=0$. The hypercomplex wavelet transforms of the other three single quadrant supported signals follow from similar computations and are given by

$$
\begin{aligned}
W_{\psi}^{(++)}\left(\boldsymbol{\zeta} ; \widetilde{g}_{-\theta, \theta}^{(-+)}\right) & =2\left[1+\operatorname{sgn}\left(\left[\boldsymbol{r}_{-\theta} \mathbf{f}_{b}\right]_{2}\right)\right]\left[1+\boldsymbol{k} \operatorname{sgn}\left(\left[\boldsymbol{r}_{-\theta} \mathbf{f}_{b}\right]_{1}\right)\right] W_{\psi}^{(e e)}(\boldsymbol{\zeta}, g), \\
W_{\psi}^{(++)}\left(\boldsymbol{\zeta} ; g_{-\theta, \theta}^{(+-)}\right) & =2\left[1+\operatorname{sgn}\left(\left[\boldsymbol{r}_{-\theta} \mathbf{f}_{b}\right]_{2}\right)\right]\left[1-\boldsymbol{k} \operatorname{sgn}\left(\left[\boldsymbol{r}_{-\theta} \mathbf{f}_{b}\right]_{1}\right)\right] W_{\psi}^{(e e)}(\boldsymbol{\zeta}, g), \\
W_{\psi}^{(++)}\left(\boldsymbol{\zeta} ; g_{-\theta, \theta}^{(--)}\right) & =-2\left[1+\operatorname{sgn}\left(\left[\boldsymbol{r}_{-\theta} \mathbf{f}_{b}\right]_{2}\right)\right]\left[1+\boldsymbol{k} \operatorname{sgn}\left(\left[\boldsymbol{r}_{-\theta} \mathbf{f}_{b}\right]_{1}\right)\right] W_{\psi}^{(e e)}(\boldsymbol{\zeta}, g) .
\end{aligned}
$$

Thus we have considered the hypercomplex wavelet transform of the $\theta$-monogenic decomposition of the real-valued signal $g(\mathbf{x})$,

C. The Fourier Transform of the Even Wavelet Transform of the A-Hypercomplex Signal, and Coefficients Equivalences

One possible strategy to construct an interpretable split into amplitude and phase descriptions would be to first construct the $\theta$-hypercomplex extension of $\widetilde{g}_{-\theta}(\mathbf{x})$ and then take the real wavelet of this object to project the $\theta$ hypercomplex extension into a small region of space and spatial frequency. The object of this procedure would be to extend the aggregate of sinusoids into a suitable quaternionic extension, and then once the quaternionic extension has been projected, the projected components are well represented in polar form. It follows that:

$$
\begin{aligned}
w_{\psi}^{(e e)}\left(\boldsymbol{\xi} ; \widetilde{g}_{-\theta, \theta}^{(++)}\right)= & w_{\psi}^{(e e)}\left(\boldsymbol{\xi} ; \widetilde{g}_{-\theta, \theta}\right)+\boldsymbol{i} w_{\psi}^{(e e)}\left(\boldsymbol{\xi} ; \widetilde{g}_{-\theta, \theta ; H}^{(1)}\right)+\boldsymbol{j} w_{\psi}^{(e e)}\left(\boldsymbol{\xi} ; \widetilde{g}_{-\theta, \theta ; H}^{(2)}\right)+\boldsymbol{k} w_{\psi}^{(e e)}\left(\boldsymbol{\xi} ; \widetilde{g}_{-\theta, \theta ; H}^{(3)}\right) \\
W_{\psi}^{(e e)}\left(\boldsymbol{\zeta} ; \widetilde{g}_{-\theta, \theta}^{(++)}\right)= & W_{\psi}^{(e e)}(\boldsymbol{\zeta} ; g)+\boldsymbol{i} W_{\psi}^{(e e)}(\boldsymbol{\zeta} ; g)(-\boldsymbol{j}) \operatorname{sgn}\left(\left[\boldsymbol{r}_{-\theta} \mathbf{f}_{b}\right]_{1}\right)+\boldsymbol{j} W_{\psi}^{(e e)}(\boldsymbol{\xi} ; g)(-\boldsymbol{j}) \operatorname{sgn}\left(\left[\boldsymbol{r}_{-\theta} \mathbf{f}_{b}\right]_{2}\right) \\
& +\boldsymbol{k} W_{\psi}^{(e e)}(\boldsymbol{\xi} ; g)(-1) \operatorname{sgn}\left(\left[\boldsymbol{r}_{-\theta} \mathbf{f}_{b}\right]_{1}\right) \operatorname{sgn}\left(\left[\boldsymbol{r}_{-\theta} \mathbf{f}_{b}\right]_{2}\right) \\
= & \left(1+\operatorname{sgn}\left(\left[\boldsymbol{r}_{-\theta} \mathbf{f}_{b}\right]_{2}\right)\right)\left(1-\boldsymbol{k} \operatorname{sgn}\left(\left[\boldsymbol{r}_{-\theta} \mathbf{f}_{b}\right]_{1}\right)\right) W_{\psi}^{(e e)}(\boldsymbol{\zeta} ; g) .
\end{aligned}
$$

We used the expression of the Fourier transform of a $\theta$-hypercomplex signal given by equation (24). 


\section{Construction of the Local $\theta$-Hypercomplex Signal} that:

Firstly let us derive an expression for $\left[W_{\psi}^{(e e)}\left(\boldsymbol{\zeta}_{0} ; \widetilde{g}_{-\theta}\right)\right]_{\theta}^{(++)}$, to show equivalence to the other expressions. Note

$$
w_{\psi}^{(e e)}\left(\boldsymbol{\xi}_{0}, \widetilde{g}_{-\theta}\right)=\int_{-\infty}^{\infty} \int_{-\infty}^{\infty} G\left(\boldsymbol{r}_{\theta} \mathbf{f}_{b}\right) a \Psi^{(e e)}\left(a \mathbf{f}_{b}\right) e^{2 \pi j \mathbf{f}_{b}^{T} \mathbf{b}} d^{2} \mathbf{f}_{b}
$$

Thus if we take the $\theta$-hypercomplex extension of $w_{\psi}^{(e e)}\left(\boldsymbol{\xi}_{0}, \widetilde{g}_{-\theta}\right)$ we obtain the representation:

$$
\begin{aligned}
{\left[w_{\psi}^{(e e)}\left(\boldsymbol{\xi}_{0}, \widetilde{g}_{-\theta}\right)\right]_{\theta}^{(++)}=} & {\left[w_{\psi}^{(e e)}\left(\boldsymbol{\xi}_{0}, \widetilde{g}_{-\theta}\right)\right]_{\theta}+\boldsymbol{i}\left[w_{\psi}^{(e e)}\left(\boldsymbol{\xi}_{0}, \widetilde{g}_{-\theta}\right)\right]_{\theta ; H}^{(1)} } \\
& +\boldsymbol{j}\left[w_{\psi}^{(e e)}\left(\boldsymbol{\xi}_{0}, \widetilde{g}_{-\theta}\right)\right]_{\theta ; H}^{(2)}+\boldsymbol{k}\left[w_{\psi}^{(e e)}\left(\boldsymbol{\xi}_{0}, \widetilde{g}_{-\theta}\right)\right]_{\theta ; H}^{(3)},
\end{aligned}
$$

We thus using equation (24) we retrieve the following in the Fourier domain:

$$
\begin{aligned}
\mathcal{F}\left\{\left[w_{\psi}^{(e e)}\left(\boldsymbol{\xi}_{0}, \widetilde{g}_{-\theta}\right)\right]_{\theta}^{(++)}\right\} & =\left[1+\operatorname{sgn}\left(\left(\boldsymbol{r}_{-\theta} \mathbf{f}_{b}\right)_{2}\right)\right]\left[1-\boldsymbol{k} \operatorname{sgn}\left(\left(\boldsymbol{r}_{-\theta} \mathbf{f}_{b}\right)_{1}\right)\right] G\left(\mathbf{f}_{b}\right) a \Psi^{(e e)}\left(a \boldsymbol{r}_{-\theta} \mathbf{f}_{b}\right) \\
& =W_{\psi}^{(++)}(\boldsymbol{\zeta} ; g),
\end{aligned}
$$

and thus we may note from equations (95,

$$
w_{\psi}^{(++)}(\boldsymbol{\xi} ; g) \equiv w_{\psi}^{(e e)}\left(\boldsymbol{\xi} ; \widetilde{g}_{-\theta, \theta}^{(++)}\right) \equiv\left[w_{\psi}^{(e e)}\left(\boldsymbol{\xi}_{0}, \widetilde{g}_{-\theta}\right)\right]_{\theta}^{(++)} .
$$

Finally as the wavelet transform is linear we may note that for any fixed value of the rotation parameter $\theta=\theta^{\prime}$, that by equation (27): $g(\mathbf{x})=\sum_{ \pm} \widetilde{g}_{-\theta^{\prime}, \theta^{\prime}}^{( \pm \pm)}(\mathbf{x})$, we retrieve:

$$
w_{\psi}^{(++)}(\boldsymbol{\xi} ; g)=\frac{1}{4}\left[w_{\psi}^{(++)}\left(\boldsymbol{\xi} ; \widetilde{g}_{-\theta^{\prime}, \theta^{\prime}}^{(++)}\right)+w_{\psi}^{(++)}\left(\boldsymbol{\xi} ; \widetilde{g}_{-\theta^{\prime}, \theta^{\prime}}^{(-+)}\right)+w_{\psi}^{(++)}\left(\boldsymbol{\xi} ; \widetilde{g}_{-\theta^{\prime}, \theta^{\prime}}^{(+-)}\right)+w_{\psi}^{(++)}\left(\boldsymbol{\xi} ; \widetilde{g}_{-\theta^{\prime}, \theta^{\prime}}^{(--)}\right] .\right.
$$

Hence we find that using equations $\mathrm{A}-18$, $\mathrm{A}-19$, $\mathrm{A}-20$ and $\mathrm{A}-21$ that the hypercomplex wavelet transform of $g(\mathbf{x})$ viewed at $\theta^{\prime}=\theta$, via equation (27) the wavelet transform of $g(\mathbf{x})$ is decomposed into the transform of each component, but that naturally sum to a hypercomplex signal:

$$
W_{\psi}^{(++)}(\boldsymbol{\zeta} ; g)=\left[1+\operatorname{sgn}\left(\left[\boldsymbol{r}_{-\theta} \mathbf{f}_{b}\right]_{2}\right)\right]\left[1-\boldsymbol{k} \operatorname{sgn}\left(\left[\boldsymbol{r}_{-\theta} \mathbf{f}_{b}\right]_{1}\right)\right] W_{\psi}^{(e e)}\left(\boldsymbol{\zeta}, \widetilde{g}_{\theta}\right) .
$$

Thus equation A-27 naturally agrees with equation 95 but by carrying out the calculation we may note that we annihilate the sum of the anti-hypercomplex components corresponding to $x_{1}$ having reversed polarity, whilst the anti-hypercomplex component corresponding to $x_{2}$ reversing sign is projected/converted into a hypercomplex signal: thus the three anti-hypercomplex components are not individually annihilated like in the 1-D case. Two of the anti-components thus cancel, whilst the third is projected into a hypercomplex object. These results will of course be necessary to derive the properties of the hypercomplex wavelet transform of the phase-shifted signal.

\section{E. 0-Hypercomplex Extension of Phase-Shifted Signal}

Note that the $\theta$-hypercomplex extension of the phase shifted signal has the FT:

$$
\begin{gathered}
\mathcal{F}\left\{\left(\Lambda_{\boldsymbol{\theta}_{s}, \theta}^{2 D} g\right)_{\theta}^{(++)}\right\}=\left(1+\operatorname{sgn}\left(\left[\boldsymbol{r}_{-\theta} \mathbf{f}\right]_{2}\right)\right)\left(1-\boldsymbol{k} \operatorname{sgn}\left(\left[\boldsymbol{r}_{-\theta} \mathbf{f}\right]_{1}\right)\right) \mathcal{F}\left\{\left(\Lambda_{\boldsymbol{\theta}_{s}, \theta}^{2 D} g\right)\right\}\left(\boldsymbol{r}_{-\theta} \mathbf{f}\right) \\
\mathcal{F}\left\{\left(\widetilde{\Lambda_{\boldsymbol{\theta}_{s}, \theta}^{2 D} g}\right)_{-\theta, \theta}^{(++)}\right\}=\left(1+\operatorname{sgn}\left(\left[\boldsymbol{r}_{-\theta} \mathbf{f}\right]_{2}\right)\right)\left(1-\boldsymbol{k} \operatorname{sgn}\left(\left[\boldsymbol{r}_{-\theta} \mathbf{f}\right]_{1}\right)\right) \mathcal{F}\left\{\left(\Lambda_{\boldsymbol{\theta}_{s}, \theta}^{2 D} g\right)\right\}(\mathbf{f}) .
\end{gathered}
$$


The FT of the separable phase-shifted signal is

$$
\begin{aligned}
& \mathcal{F}\left\{\Lambda_{\boldsymbol{\theta}_{s}, \theta}^{2 D} g\right\}(\mathbf{f})=\int_{-\infty}^{\infty} \int_{-\infty}^{\infty}\left|\widetilde{g}_{-\theta}^{(++)}\left(\boldsymbol{r}_{-\theta} \mathbf{x}\right)\right| \cos \left(2 \pi \widetilde{\alpha}_{-\theta}\left(\boldsymbol{r}_{-\theta} \mathbf{x}\right)-\theta_{s, 1}\right) \cos \left(2 \pi \widetilde{\beta}_{-\theta}\left(\boldsymbol{r}_{-\theta} \mathbf{x}\right)-\theta_{s, 2}\right) \\
& e^{-2 \pi \boldsymbol{j}\left(\boldsymbol{r}_{-\theta} \mathbf{x}\right)^{T} \boldsymbol{r}_{-\theta} \mathbf{f}} d^{2} \mathbf{x} \\
& =\frac{1}{4}\left[e^{-\boldsymbol{j} \theta_{s, 1}} \widetilde{G}_{1,-\theta}^{(+)}\left(\left[\boldsymbol{r}_{-\theta} \mathbf{f}\right]_{1}\right)+e^{j \theta_{s, 1}} \widetilde{G}_{1,-\theta}^{(-)}\left(\left[\boldsymbol{r}_{-\theta} \mathbf{f}\right]_{1}\right)\right] \\
& {\left[e^{-\boldsymbol{j} \theta_{s, 2}} \widetilde{G}_{2,-\theta}^{(+)}\left(\left[\boldsymbol{r}_{-\theta} \mathbf{f}\right]_{2}\right)+e^{\boldsymbol{j} \theta_{s, 2}} \widetilde{G}_{2,-\theta}^{(-)}\left(\left[\boldsymbol{r}_{-\theta} \mathbf{f}\right]_{2}\right)\right]} \\
& =\frac{1}{4}\left[e^{-\boldsymbol{j} \theta_{s, 1}}\left(1+\operatorname{sgn}\left(\left[\boldsymbol{r}_{-\theta} \mathbf{f}\right]_{1}\right)\right)+e^{\boldsymbol{j} \theta_{s, 1}}\left(1-\operatorname{sgn}\left(\left[\boldsymbol{r}_{-\theta} \mathbf{f}\right]_{1}\right)\right)\right] \widetilde{G}_{-\theta}\left(\boldsymbol{r}_{-\theta} \mathbf{f}\right) \\
& {\left[e^{-\boldsymbol{j} \theta_{s, 2}}\left(1+\operatorname{sgn}\left(\left[\boldsymbol{r}_{-\theta} \mathbf{f}\right]_{2}\right)\right)+e^{\boldsymbol{j} \theta_{s, 2}}\left(1-\operatorname{sgn}\left(\left[\boldsymbol{r}_{-\theta} \mathbf{f}\right]_{2}\right)\right)\right] \text {. }}
\end{aligned}
$$

After some algebra we then obtain:

$$
\begin{aligned}
\mathcal{F}\left\{\left(\widetilde{\Lambda_{\boldsymbol{\theta}_{s}, \theta}^{2 D} g}\right)_{-\theta, \theta}^{(++)}\right\}(\mathbf{f})= & \left(1+\operatorname{sgn}\left(\left[\boldsymbol{r}_{-\theta} \mathbf{f}\right]_{2}\right)\right)\left(1-\boldsymbol{k} \operatorname{sgn}\left(\left[\boldsymbol{r}_{-\theta} \mathbf{f}\right]_{1}\right)\right) \\
& \frac{1}{4}\left[e^{-\boldsymbol{j} \theta_{s, 1}}\left(1+\operatorname{sgn}\left(\left[\boldsymbol{r}_{-\theta} \mathbf{f}\right]_{1}\right)\right)+e^{\boldsymbol{j} \theta_{s, 1}}\left(1-\operatorname{sgn}\left(\left[\boldsymbol{r}_{-\theta} \mathbf{f}\right]_{1}\right)\right)\right] \widetilde{G}_{-\theta}\left(\boldsymbol{r}_{-\theta} \mathbf{f}\right) \\
& {\left[e^{-\boldsymbol{j} \theta_{s, 2}}\left(1+\operatorname{sgn}\left(\left[\boldsymbol{r}_{-\theta} \mathbf{f}\right]_{2}\right)\right)+e^{\boldsymbol{j} \theta_{s, 2}}\left(1-\operatorname{sgn}\left(\left[\boldsymbol{r}_{-\theta} \mathbf{f}\right]_{2}\right)\right)\right] } \\
= & e^{-\boldsymbol{i} \theta_{s, 1}} \widetilde{G}_{-\theta, \theta}^{(++)}(\mathbf{f}) e^{-\boldsymbol{j} \theta_{s, 2}} .
\end{aligned}
$$

Hence we may deduce that, as would be expected for $g(\mathbf{x})$ a separable signal we determine that:

$$
\left.\widetilde{\left(\Lambda_{\boldsymbol{\theta}_{s}, \theta}^{2 D} g\right.}\right)_{-\theta, \theta}^{(++)}(\mathbf{x})=e^{-\boldsymbol{i} \theta_{s, 1}} \widetilde{g}_{-\theta, \theta}^{(++)}(\mathbf{x}) e^{-\boldsymbol{j} \theta_{s, 2}} .
$$

\section{F. Hypercomplex CWT of a Phase-Shifted Signal}

We note that the hypercomplex CWT of the observed signal $g(\mathbf{x})$ is by equations $\mathrm{A}-26$ and $\mathrm{A}-28$ :

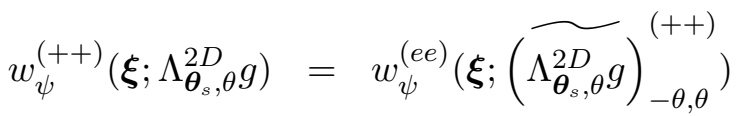

$$
\begin{aligned}
& =e^{-\boldsymbol{i} \theta_{s, 1}} w_{\psi}^{(e e)}\left(\boldsymbol{\xi} ; \widetilde{g}_{-\theta, \theta}^{(++)}(\mathbf{x})\right) e^{-\boldsymbol{j} \theta_{s, 2}} \\
& =e^{-\boldsymbol{i} \theta_{s, 1}} w_{\psi}^{(++)}(\boldsymbol{\xi} ; g) e^{-\boldsymbol{j} \theta_{s, 2}},
\end{aligned}
$$

from equation $\mathrm{A}-28$. Thus the result follows.

\section{G. FT and QFT of the Monogenic Wavelet Transform}

Recalling equations (37), (92) as well as (92) we obtain the FT

$$
\begin{aligned}
W_{\psi}^{(+)}(\boldsymbol{\zeta} ; g) & =W_{\psi}^{(r)}(\boldsymbol{\zeta} ; g)-\boldsymbol{i} W_{\psi}^{(1)}(\boldsymbol{\zeta} ; g)-\boldsymbol{j} W_{\psi}^{(2)}(\boldsymbol{\zeta} ; g) \\
& =\left[1-\boldsymbol{k} \cos \left(\phi_{b}-\theta\right)+\sin \left(\phi_{b}-\theta\right)\right] W_{\psi}^{(r)}(\boldsymbol{\zeta} ; g) .
\end{aligned}
$$

The corresponding QFT is

$$
\begin{aligned}
W_{\psi ; Q}^{(+)}(\boldsymbol{\kappa} ; g) & =W_{\psi ; Q}^{(r)}(\boldsymbol{\kappa} ; g)-i W_{\psi ; Q}^{(1)}(\boldsymbol{\kappa} ; g)-W_{\psi ; Q}^{(2)}(\boldsymbol{\kappa} ; g) \boldsymbol{j} \\
& =\frac{1-\boldsymbol{k}}{2}\left[1+\cos \left(\nu_{b}-\theta\right)+\sin \left(\nu_{b}-\theta\right)\right] G\left(\mathbf{q}_{b}\right) a \Psi^{(r) *}\left(a \boldsymbol{r}_{-\theta} \mathbf{q}_{b}\right) \\
& +\frac{1+\boldsymbol{k}}{2}\left[1+\cos \left(\nu_{b}+\theta\right)+\sin \left(\nu_{b}+\theta\right)\right] G\left(\boldsymbol{J}_{\pi / 2} \mathbf{q}_{b}\right) a \Psi^{(r) *}\left(a \boldsymbol{r}_{-\theta} \boldsymbol{J}_{\pi / 2} \mathbf{q}_{b}\right) \\
& =W_{\psi ; Q}^{(r)}(\boldsymbol{\kappa} ; g)+\cos \theta\left[\cos \nu_{b}+\sin \nu_{b}\right] W_{\psi ; Q}^{(r)}(\boldsymbol{\kappa} ; g)+\sin \theta\left[\cos \nu_{b}-\sin \nu_{b}\right] \boldsymbol{i} W_{\psi ; Q}^{(r)}(\boldsymbol{\kappa} ; g) \boldsymbol{j} .
\end{aligned}
$$




\section{H. Wavelet transform of $\theta$-Monogenic \& Anti-Monogenic Decomposition Components}

We find that the Fourier transform of $\widetilde{g}_{-\theta^{\prime}, \theta^{\prime}}^{(+)}(\mathbf{x})$ is given by:

$$
\begin{aligned}
& W_{\psi}^{(+)}\left(\boldsymbol{\zeta} ; \widetilde{g}_{-\theta^{\prime}, \theta^{\prime}}^{(+)}\right)=W_{\psi}^{(+)}(\boldsymbol{\zeta} ; g)+\left[\boldsymbol{i} W_{\psi}^{(+)}\left(\boldsymbol{\zeta} ; \widetilde{g}_{-\theta^{\prime}, \theta^{\prime}, R}^{(1)}\right)+\boldsymbol{j} W_{\psi}^{(+)}\left(\boldsymbol{\zeta} ; \widetilde{g}_{-\theta^{\prime}, \theta^{\prime}, R}^{(2)}\right)\right] \\
& =\left[1+\sin \left(\phi_{b}-\theta\right)+\cos \left(\phi_{b}-\theta\right) \cos \left(\phi_{b}-\theta^{\prime}\right)+\sin \left(\phi_{b}-\theta^{\prime}\right)+\sin \left(\phi_{b}-\theta\right) \sin \left(\phi_{b}-\theta^{\prime}\right)\right. \\
& \left.+\boldsymbol{k}\left(-\cos \left(\phi_{b}-\theta\right)-\cos \left(\phi_{b}-\theta^{\prime}\right)-\sin \left(\phi_{b}-\theta\right) \cos \left(\phi_{b}-\theta^{\prime}\right)+\cos \left(\phi_{b}-\theta\right) \sin \left(\phi_{b}-\theta^{\prime}\right)\right)\right] W_{\psi}^{(r)}(\boldsymbol{\zeta} ; g) .
\end{aligned}
$$

We remark that with $\theta=\theta^{\prime}$ this then becomes

$$
\begin{aligned}
W_{\psi}^{(+)}\left(\boldsymbol{\zeta} ; \widetilde{g}_{-\theta, \theta}^{(+)}\right) & =\left\{2+2 \sin \left(\phi_{b}-\theta\right)-2 \boldsymbol{k} \cos \left(\phi_{b}-\theta\right)\right\} W_{\psi}^{(r)}(\boldsymbol{\zeta} ; g) \\
& =\left\{2+2 \sin \left(\phi_{b}-\theta\right)-2 \boldsymbol{k} \cos \left(\phi_{b}-\theta\right)\right\} G\left(\mathbf{f}_{b}\right) a \Psi^{(r) *}\left(a \boldsymbol{r}_{-\theta} \mathbf{f}_{b}\right) \\
& =\left\{2+2 \sin \left(\phi_{b}-\theta\right)-2 \boldsymbol{k} \cos \left(\phi_{b}-\theta\right)\right\} \widetilde{G}_{-\theta, \theta}\left(\mathbf{f}_{b}\right) a \Psi^{(r) *}\left(a \boldsymbol{r}_{-\theta} \mathbf{f}_{b}\right) \\
& =2 \widetilde{G}_{-\theta, \theta}^{(+)}\left(\mathbf{f}_{b}\right) a \Psi^{(r) *}\left(a \boldsymbol{r}_{-\theta} \mathbf{f}_{b}\right)=2 W_{\psi}^{(r)}\left(\boldsymbol{\zeta} ; \widetilde{g}_{-\theta, \theta}^{(+)}\right) .
\end{aligned}
$$

Furthermore mutatis mutandis with $\widetilde{g}_{-\theta, \theta}^{(-)}(\mathbf{x})$ replacing $\widetilde{g}_{-\theta, \theta}^{(+)}(\mathbf{x})$ we derive that

$$
W_{\psi}^{(+)}\left(\boldsymbol{\zeta} ; \widetilde{g}_{-\theta, \theta}^{(-)}\right)=0 .
$$

This completes the proof. We can note that equation ( $\mathrm{A}-31$ establishes the necessity of introducing the $\theta$-monogenic signal. The Monogenic wavelet transform will not annihilate the monogenic signal for example, and in the subsequent section this may produce problems.

\section{Construction of Local A-Monogenic Signal}

We note the form of the Fourier transform of $w_{\psi}^{(+)}(\boldsymbol{\xi} ; g)$ from equation (106) and then from equation (42) we find that (recalling $\boldsymbol{\xi}_{0}=[a, 0, \mathbf{b}]^{T}$ ):

$$
\begin{aligned}
\mathcal{F}\left\{\left[w_{\psi}^{(r)}\left(\boldsymbol{\xi}_{0} ; \widetilde{g}_{-\theta}\right)\right]_{\theta}^{(+)}\right\} & =\left[1+\sin \left(\phi_{b}-\theta\right)-\boldsymbol{k} \cos \left(\phi_{b}-\theta\right)\right] \widetilde{G}_{-\theta}\left(\boldsymbol{r}_{-\theta} \mathbf{f}_{b}\right) a \Psi^{(r) *}\left(a \boldsymbol{r}_{-\theta} \mathbf{f}_{b}\right) \\
& =\left[1+\sin \left(\phi_{b}-\theta\right)-\boldsymbol{k} \cos \left(\phi_{b}-\theta\right)\right] G\left(\mathbf{f}_{b}\right) a \Psi^{(r) *}\left(a \boldsymbol{r}_{-\theta} \mathbf{f}_{b}\right) \\
& =W_{\psi}^{(+)}(\boldsymbol{\zeta} ; g) \\
{\left[w_{\psi}^{(r)}\left(\boldsymbol{\xi}_{0} ; \widetilde{g}_{-\theta}\right)\right]_{\theta}^{(+)} } & =w_{\psi}^{+}(\boldsymbol{\xi} ; g) .
\end{aligned}
$$

Thus the monogenic wavelet transform coefficients correspond to the monogenic extension of the real wavelet transform in the rotated frame of reference. For the second equality we start by using equation (45) and take

$$
g(\mathbf{x})=\frac{1}{2}\left(\widetilde{g}_{-\theta^{\prime}, \theta^{\prime}}^{(+)}(\mathbf{x})+\widetilde{g}_{-\theta^{\prime}, \theta^{\prime}}^{(-)}(\mathbf{x})\right) .
$$

By the linearity of the wavelet transform we have that with $\theta^{\prime}=\theta$ using equations $\mathrm{A}-32$ and $\mathrm{A}-33$ :

$$
\begin{aligned}
W_{\psi}^{(+)}(\boldsymbol{\zeta} ; g) & =\frac{1}{2}\left[W_{\psi}^{(+)}\left(\boldsymbol{\zeta} ; \widetilde{g}_{-\theta, \theta}^{(+)}\right)+W_{\psi}^{(+)}\left(\boldsymbol{\zeta} ; \widetilde{g}_{-\theta, \theta}^{(-)}\right)\right] \\
& =\frac{1}{2}\left[2 W_{\psi}^{(r)}\left(\boldsymbol{\zeta} ; \widetilde{g}_{-\theta, \theta}^{(+)}\right)+0\right]=W_{\psi}^{(r)}\left(\boldsymbol{\zeta} ; \widetilde{g}_{-\theta, \theta}^{(+)}\right),
\end{aligned}
$$

and thus the result follows. Hence the operation of constructing the monogenic wavelet transform can either be considered in light of the operation of 1) taking the monogenic wavelet transform of a real signal, 2) finding the real wavelet transform of a $\theta$-monogenic signal or as finding the $\theta$-monogenic image of the wavelet transform in $\mathbf{b}$ of the rotated by $\theta$ signal. 


\section{J. Local Invariance under Rotation}

If the monogenic wavelet is constructed from an isotropic wavelet, then it follows that:

$$
\begin{aligned}
w_{\psi}^{(e)}(\boldsymbol{\xi} ; g) & =\int_{-\infty}^{\infty} \int_{-\infty}^{\infty} W_{\psi}^{(e)}(\boldsymbol{\zeta} ; g) e^{2 \boldsymbol{j} \pi \mathbf{f}_{b}^{T} \mathbf{b}} d^{2} \mathbf{f}_{b}=\int_{-\infty}^{\infty} \int_{-\infty}^{\infty} G\left(\mathbf{f}_{b}\right) a \Psi\left(a \boldsymbol{r}_{-\theta} \mathbf{f}_{b}\right) e^{2 \boldsymbol{j} \pi \mathbf{f}_{b}^{T} \mathbf{b}} d^{2} \mathbf{f}_{b} \\
& \stackrel{(1)}{=} \int_{-\infty}^{\infty} \int_{-\infty}^{\infty} G\left(\mathbf{f}_{b}\right) a \Psi\left(a \mathbf{f}_{b}\right) e^{2 \boldsymbol{j} \pi \mathbf{f}_{b}^{T} \mathbf{b}} d^{2} \mathbf{f}_{b}=\int_{-\infty}^{\infty} \int_{-\infty}^{\infty} W_{\psi}^{(e)}\left(\boldsymbol{\zeta}_{0} ; g\right) e^{2 \boldsymbol{j} \pi \mathbf{f}_{b}^{T} \mathbf{b}} d^{2} \mathbf{f}_{b} \\
& =w_{\psi}^{(e)}\left(\boldsymbol{\xi}_{0} ; g\right),
\end{aligned}
$$

where we have used that the real mother wavelet is isotropic in (1). In a similar fashion, we calculate the for the CWTs with respect to the first and second Riesz components of the monogenic wavelet,

$$
\begin{aligned}
& w_{\psi}^{(1)}(\boldsymbol{\xi} ; g)=\cos (\theta) w_{\psi}^{(1)}\left(\boldsymbol{\xi}_{0} ; g\right)+\sin (\theta) w_{\psi}^{(2)}\left(\boldsymbol{\xi}_{0} ; g\right), \\
& w_{\psi}^{(2)}(\boldsymbol{\xi} ; g)=-\sin (\theta) w_{\psi}^{(1)}\left(\boldsymbol{\xi}_{0} ; g\right)+\cos (\theta) w_{\psi}^{(2)}\left(\boldsymbol{\xi}_{0} ; g\right) .
\end{aligned}
$$

Using the above expressions $\mathrm{A}-35$, and $\mathrm{A}-36$, trivially follows that

$$
\left|w_{\psi}^{(+)}(\boldsymbol{\xi} ; g)\right|^{2}=w_{\psi}^{(r) 2}(\boldsymbol{\xi} ; g)+w_{\psi}^{(1) 2}(\boldsymbol{\xi} ; g)+w_{\psi}^{(2) 2}(\boldsymbol{\xi} ; g)=\left|w_{\psi}^{(+)}\left(\boldsymbol{\xi}_{0} ; g\right)\right|^{2} \text {. }
$$

Hence, if an isotropic real mother wavelet is used, the magnitude of the monogenic wavelet coefficients is invariant to to the choice of orientation.

\section{K. Directional Selectivity}

For simplicity we model the signal is directional in the Fourier domain, i.e. via equation (114). Note that the wavelet transform corresponds to patterns at period $f$. It then transpires that:

$$
\begin{aligned}
w_{\psi}^{(1)}(\boldsymbol{\xi} ; g)= & \int_{-\infty}^{\infty} \int_{-\infty}^{\infty} W_{\psi}^{(1)}(\boldsymbol{\zeta} ; g) e^{2 \boldsymbol{j} \pi \mathbf{f}_{b}^{T} \mathbf{b}} d^{2} \mathbf{f}_{b} \\
\stackrel{(1)}{=} & \int_{0}^{\infty} \int_{0}^{2 \pi}(-\boldsymbol{j}) f_{b} \cos \left(\phi_{b}-\theta\right) \frac{\widetilde{G}\left(f_{b}\right)}{2}\left[\delta\left(\phi_{b}-\phi_{0}-\pi\right)+\delta\left(\phi_{b}-\phi_{0}\right)\right] a \Psi^{(e)}\left(a f_{b}\right) \\
& e^{2 \boldsymbol{j} \pi \mathbf{f}_{b}^{T} \mathbf{b}} d f_{b} d \phi \\
= & \cos \left(\theta-\phi_{0}\right) \int_{0}^{\infty} f_{b} \widetilde{G}\left(f_{b}\right) a \Psi^{(e)}(a f) \sin \left(2 \pi f_{b} b \cos \left(\chi_{b}-\phi_{0}\right)\right) d f_{b},
\end{aligned}
$$

In a similar fashion,

$$
w_{\psi}^{(2)}(\boldsymbol{\xi} ; g)=\sin \left(\theta-\phi_{0}\right) \int_{0}^{\infty} f_{b} \widetilde{G}\left(f_{b}\right) a \Psi^{(e)}\left(a f_{b}\right) \sin \left(2 \pi f_{b} b \cos \left(\chi_{b}-\phi_{0}\right)\right) d f_{b} .
$$

For (1) to hold, $\psi^{(r)}(\mathbf{x}) \equiv \psi^{(e)}(\mathbf{x})$, i.e. is isotropic. For a directional signal, when $w_{\psi}^{(1)}(\boldsymbol{\xi} ; g) \neq 0$,

$$
\theta-\phi_{0}=\tan ^{-1}\left(\frac{w_{\psi}^{(2)}(\boldsymbol{\xi} ; g)}{w_{\psi}^{(1)}(\boldsymbol{\xi} ; g)}\right) .
$$

Thus the local directionality of a directional signal, can at any rotational angle of the wavelet transform, be determined from the isotropic monogenic wavelet transform. Furthermore it is clear that

$$
w_{\psi}^{(2)}\left(\boldsymbol{\xi}^{\dagger} ; g\right)=0
$$

and hence by equation 107, the stated result follows. 


\section{CWT of Phase-Shifted Signal}

For brevity we denote the wavelet transform of the phase-shifted signals by $w_{\psi, \theta_{s}}^{( \pm)}(\boldsymbol{\xi} ; g)=w_{\psi}^{( \pm)}\left(\boldsymbol{\xi} ; \Lambda_{\theta_{s}} g\right)$. We note that

$$
\begin{aligned}
& \Lambda_{\theta_{s}} g(\mathbf{x})=\left|g^{(+)}(\mathbf{x})\right| \cos \left(2 \pi \phi(\mathbf{x})-\theta_{s}\right) \\
& =\frac{1}{2}\left[e^{-\boldsymbol{e}_{\tilde{\nu}_{-\theta, \theta}}(\mathbf{x}) \theta_{s}} \widetilde{g}_{-\theta, \theta}^{(+)}(\mathbf{x})+e^{\boldsymbol{e}_{\tilde{\nu}_{-\theta, \theta}}(\mathbf{x}) \theta_{s}} \widetilde{g}_{-\theta, \theta}^{(-)}(\mathbf{x})\right] .
\end{aligned}
$$

Hence with the additional assumption that $\boldsymbol{e}_{\widetilde{\nu}_{-\theta, \theta}}(\mathbf{x})=\boldsymbol{e}_{\widetilde{\nu}_{-\theta, \theta}}(\mathbf{b})$ is constant over the spatial width the wavelet transform is averaging over, i.e. $|\mathbf{x}-\mathbf{b}|<a x_{i}$ we retrieve that

$$
\begin{aligned}
& w_{\psi, \theta_{s}}^{(+)}(\boldsymbol{\xi} ; g)=\frac{1}{2}\left[w_{\psi}^{(+)}\left(\boldsymbol{\xi} ; e^{-\boldsymbol{e}_{\tilde{\nu}_{-\theta}, \theta}(\mathbf{x}) \theta_{s}} \widetilde{g}_{-\theta, \theta}^{(+)}(\mathbf{x})\right)+w_{\psi}^{(+)}\left(\boldsymbol{\xi} ; e^{\boldsymbol{e}_{\tilde{\nu}_{-\theta}, \theta}(\mathbf{x}) \theta_{s}} \widetilde{g}_{-\theta, \theta}^{(-)}(\mathbf{x})\right)\right] \\
& =\frac{1}{2}\left[w_{\psi}^{(+)}\left(\boldsymbol{\xi} ; e^{-\boldsymbol{e}_{\tilde{\nu}_{-\theta, \theta}}(\mathbf{b}) \theta_{s}} \widetilde{g}_{-\theta, \theta}^{(+)}(\mathbf{x})\right)+w_{\psi}^{(+)}\left(\boldsymbol{\xi} ; e^{\boldsymbol{e}_{\tilde{\nu}_{-\theta, \theta}}(\mathbf{b}) \theta_{s}} \widetilde{g}_{-\theta, \theta}^{(-)}(\mathbf{x})\right)\right] \\
& =\frac{1}{2}\left[e^{-\boldsymbol{e}_{\widetilde{\nu}_{-\theta, \theta}}(\mathbf{b}) \theta_{s}} w_{\psi}^{(+)}\left(\boldsymbol{\xi} ; \widetilde{g}_{-\theta, \theta}^{(+)}(\mathbf{x})\right)+e^{\boldsymbol{e}_{\widetilde{\nu}_{-\theta, \theta}}(\mathbf{b}) \theta_{s}} w_{\psi}^{(+)}\left(\boldsymbol{\xi} ; \widetilde{g}_{-\theta, \theta}^{(-)}(\mathbf{x})\right)\right] \\
& =\frac{1}{2} e^{-\boldsymbol{e}_{\tilde{\nu}_{-\theta, \theta}}(\mathbf{b}) \theta_{s}} w_{\psi}^{(+)}\left(\boldsymbol{\xi} ; \widetilde{g}_{-\theta, \theta}^{(+)}(\mathbf{x})\right)=e^{-\boldsymbol{e}_{\tilde{\nu}_{-\theta, \theta}}(\mathbf{b}) \theta_{s}} w_{\psi}^{(+)}(\boldsymbol{\xi} ; g(\mathbf{x})),
\end{aligned}
$$

where we used equation $\mathrm{A}-33$. Also from equation $\mathrm{A}-32$ we may note

$$
w_{\psi}^{(+)}(\boldsymbol{\xi} ; g(\mathbf{x}))=\frac{1}{2} w_{\psi}^{(+)}\left(\boldsymbol{\xi} ; \widetilde{g}_{\theta}^{(+)}(\mathbf{x})\right)
$$

and thus the result follows. 